\title{
SUITABILITY OF FINITE STATE AUTOMATA TO MODEL STRING CONSTRAINTS IN PROBABILISTIC SYMBOLIC EXECUTION
}

by

Andrew Harris

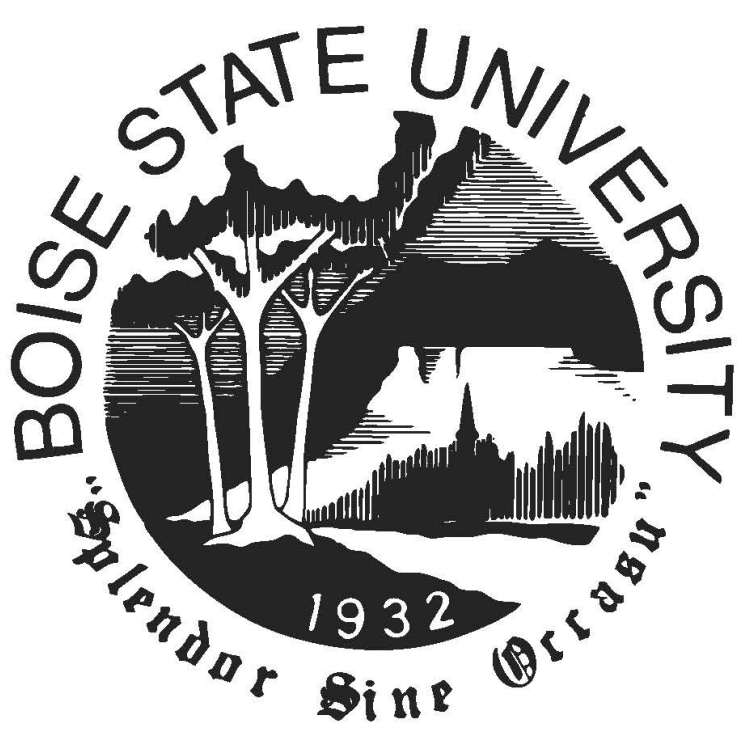

A thesis

submitted in partial fulfillment

of the requirements for the degree of

Master of Science in Computer Science

Boise State University

August 2019 
(C) 2019

Andrew Harris

ALL RIGHTS RESERVED 
BOISE STATE UNIVERSITY GRADUATE COLLEGE

DEFENSE COMMITTEE AND FINAL READING APPROVALS

of the thesis submitted by

\author{
Andrew Harris
}

Thesis Title: Suitability of Finite State Automata to Model String Constraints in Probabilistic Symbolic Execution

Date of Final Oral Examination: 5th May 2017

The following individuals read and discussed the thesis submitted by student Andrew Harris, and they evaluated their presentation and response to questions during the final oral examination. They found that the student passed the final oral examination.

Elena Sherman, Ph.D. Chair, Supervisory Committee

Jim Buffenbarger, Ph.D. Member, Supervisory Committee

Bogdan Dit, Ph.D. Member, Supervisory Committee

The final reading approval of the thesis was granted by Elena Sherman, Ph.D., Chair, Supervisory Committee. The thesis was approved by the Graduate College. 


\section{ACKNOWLEDGMENTS}

The author wishes to express gratitude to the National Science Foundation who's grant supported a portion of this work. 


\begin{abstract}
Probabilistic Symbolic Execution (PSE) extends Symbolic Execution (SE), a pathsensitive static program analysis technique, by calculating the probabilities with which program paths are executed. PSE relies on the ability of the underlying symbolic models to accurately represent the execution paths of the program as the collection of input values following these paths. While researchers established PSE for numerical data types, PSE for complex data types such as strings is a novel area of research.

For string data types SE tools commonly utilize finite state automata to represent a symbolic string model. Thus, PSE inherits from SE automata-based symbolic string models to calculate the probabilities of string-based constraints describing program paths. However, to our knowledge, there is lack of research on suitability of automatabased symbolic string models in the context of PSE.

This thesis proposes four automata-based symbolic string models for PSE and analyzes their suitability using two criteria: accuracy and performance. We compare the probability computed by the model to the actual probability and the amount of time took to compute it. Our results show that each model varies in their accuracy, however none is able to consistently compute actual value. In addition, our evaluation did reveal that this amount of inaccuracy depends upon the characteristics of a software program. From these findings we suggest guidance when selecting an automaton model for PSE based on the performance and accuracy requirements and the characteristics of the program under analysis. Additionally, we suggest future areas of research to the accuracy and performance deficiencies observed in our evaluation.
\end{abstract}




\section{TABLE OF CONTENTS}

ABSTRACT $\ldots \ldots \ldots \ldots \ldots \ldots \ldots \ldots \ldots \ldots \ldots \ldots \ldots \ldots \ldots \ldots$

LIST OF TABLES $\ldots \ldots \ldots \ldots \ldots \ldots \ldots \ldots \ldots \ldots \ldots \ldots \ldots \ldots \ldots \ldots$

LIST OF FIGURES $\ldots \ldots \ldots \ldots \ldots \ldots \ldots \ldots \ldots \ldots \ldots \ldots \ldots \ldots \ldots$ xii

LIST OF ABBREVIATIONS $\ldots \ldots \ldots \ldots \ldots \ldots \ldots \ldots \ldots \ldots$ xvi

LIST OF SYMBOLS $\ldots \ldots \ldots \ldots \ldots \ldots \ldots \ldots \ldots \ldots \ldots \ldots \ldots \ldots \ldots$

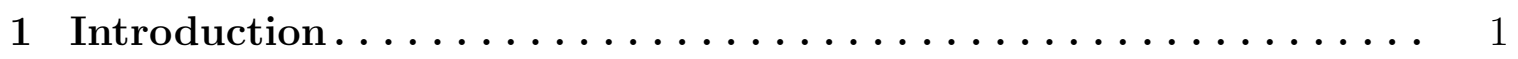

1.1 Quantitative String Analysis $\ldots \ldots \ldots \ldots \ldots \ldots \ldots \ldots \ldots \ldots$

1.2 Example .............................. 2

1.3 Symbolic String Models $\ldots \ldots \ldots \ldots \ldots \ldots \ldots \ldots \ldots \ldots \ldots$

1.4 Thesis Statement . . . . . . . . . . . . . . . . . . . . 4

1.5 Contributions ........................... 5

2 Background $\ldots \ldots \ldots \ldots \ldots \ldots \ldots \ldots \ldots \ldots \ldots \ldots \ldots \ldots \ldots \ldots \ldots \ldots \ldots$

2.1 Symbolic Execution $\ldots \ldots \ldots \ldots \ldots \ldots \ldots \ldots \ldots \ldots \ldots \ldots \ldots$

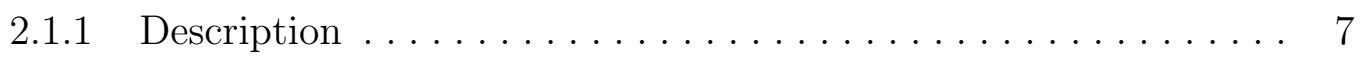

2.1.2 Example ............................ 10

2.1 .3 Limitations . . . . . . . . . . . . . . . . . . 11

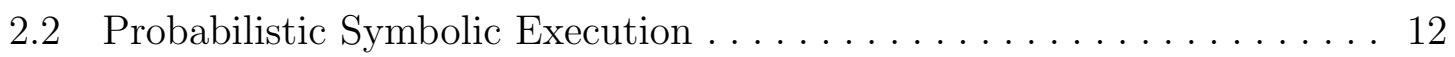


$2.2 .1 \quad$ Description $\ldots \ldots \ldots \ldots \ldots \ldots \ldots \ldots \ldots \ldots \ldots \ldots \ldots$

$2.2 .2 \quad$ Example ............................ 15

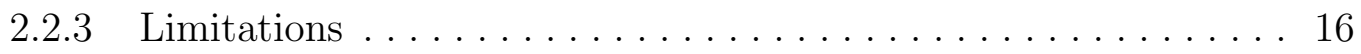

2.3 Modeling String Constraints . . . . . . . . . . . . . . . . 20

2.3.1 Symbolic String Models $\ldots \ldots \ldots \ldots \ldots \ldots \ldots \ldots \ldots \ldots$. . . . . . 21

2.3.2 Limitations . . . . . . . . . . . . . . . . . . . . . . . . 23

2.4 Modeling String Constraints with Finite State Automata . . . . . . . . 25

2.4.1 Finite State Automata . . . . . . . . . . . . . . . 26

2.4.2 Automata as Symbolic String Models . . . . . . . . . . . . . 29

2.4 .3 Limitations . . . . . . . . . . . . . . . . . . . 44

3 Automata-based Symbolic String Models . . . . . . . . . . . . . . 46

3.1 Unbounded Automata Model . . . . . . . . . . . . . . . . . . . 47

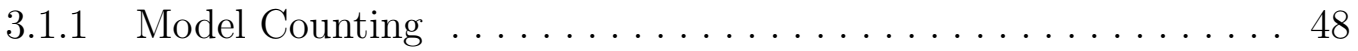

3.1 .2 Over-Approximation ..................... 50

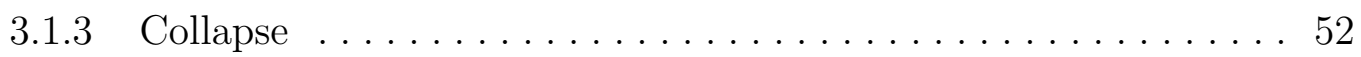

3.2 Bounded Automata Model . . . . . . . . . . . . . . . . . . . . . . . 55

3.2 .1 Model Counting . . . . . . . . . . . . . . . . . . . . . . 56

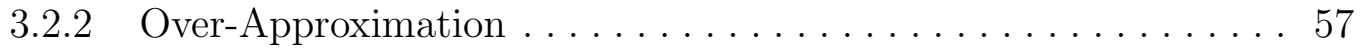

3.2 .3 Collapse $\ldots \ldots \ldots \ldots \ldots \ldots \ldots \ldots \ldots \ldots \ldots \ldots \ldots$

3.3 Aggregate Bounded Automata Model . . . . . . . . . . . . . . . . 60

3.3 .1 String Operations $\ldots \ldots \ldots \ldots \ldots \ldots \ldots \ldots \ldots \ldots \ldots \ldots$

3.3 .2 Model Counting $\ldots \ldots \ldots \ldots \ldots \ldots \ldots \ldots \ldots \ldots$

3.3 .3 Over-Approximation .......................6 67

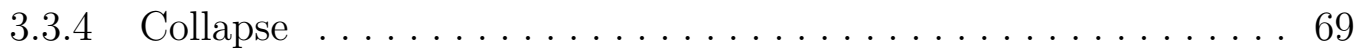


3.4 Weighted Transition Automata Model . . . . . . . . . . . . . . . . 72

3.4.1 Weighted-Transition Finite State Automaton ............. . 73

3.4 .2 Model Creation . . . . . . . . . . . . . . . . . . . . 74

3.4 .3 Model Counting $\ldots \ldots \ldots \ldots \ldots \ldots \ldots \ldots \ldots \ldots \ldots$

3.4.4 String Operations and Predicates $\ldots \ldots \ldots \ldots \ldots \ldots \ldots$

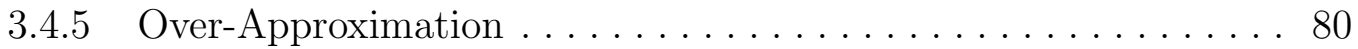

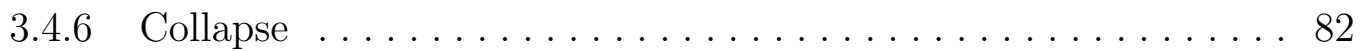

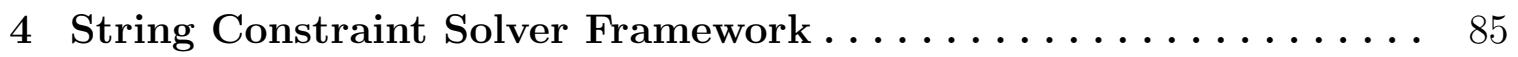

4.1 Third Party Dependencies $\ldots \ldots \ldots \ldots \ldots \ldots \ldots \ldots \ldots \ldots$

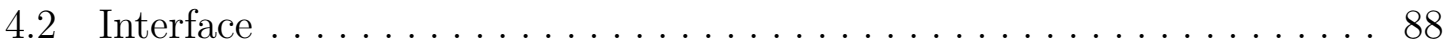

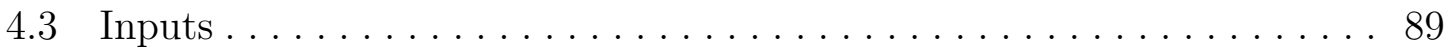

4.3.1 Constraint Graph Structure . . . . . . . . . . . . . . . 90

4.3.2 Synthetic Constraint Graphs . . . . . . . . . . . . . . . . 92

4.4 Components . . . . . . . . . . . . . . . . . . . . . 93

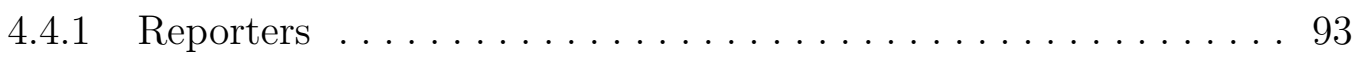

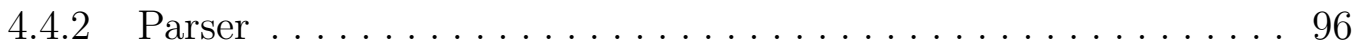

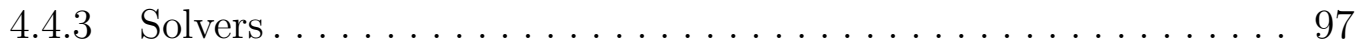

4.4.4 Automaton Models ............................... 99

4.4.5 Weighted-Transition Finite State Automaton .... . . . . . . . 102

4.4.6 Symbolic String Model Algorithms . . . . . . . . . . . . . . . . 103

4.5 Test Suite . . . . . . . . . . . . . . . . . . . . . . 105

$4.6 \quad$ Utility Scripts . . . . . . . . . . . . . . . . . . . . . 105

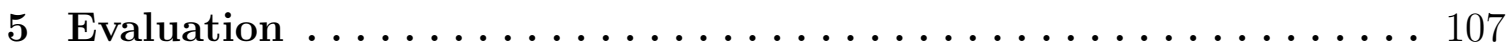

$5.1 \quad$ Evaluation Procedure ............................ 108 
5.1 .1 Constraint Generation . . . . . . . . . . . . . . . . . . . 108

5.1 .2 Constraint Solving . . . . . . . . . . . . . . . . . . . . 109

5.1 .3 Data Collection . . . . . . . . . . . . . . . . . . . . 110

5.1 .4 Data Analysis . . . . . . . . . . . . . . . . . . . . . . 112

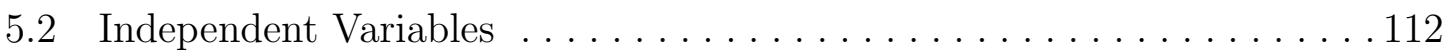

5.2 .1 String Alphabet . . . . . . . . . . . . . . . . . . . . . 113

5.2 .2 Initial String Length . . . . . . . . . . . . . . . . . . . . . 114

5.2 .3 Input String Type . . . . . . . . . . . . . . . . . . . . 114

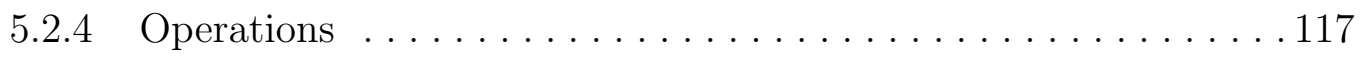

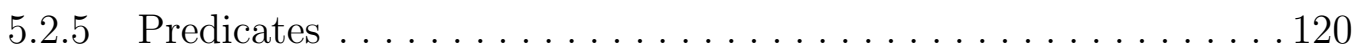

5.2.6 Distribution of Independent Variables in Graphs . . . . . . . . 121

5.3 Accuracy . . . . . . . . . . . . . . . . . . . . . . . . . 123

5.3.1 Oracle / ConcreteSolver . . . . . . . . . . . . . . . . . . . . 124

$5.3 .2 \quad$ Measurement............................... 124

5.3.3 Effects of Independent Variables . . . . . . . . . . . . . . 126

5.4 Performance ................................ 126

5.4.1 Constraint Solving Performance ................... 127

5.4.2 Model Counting Performance . . . . . . . . . . . . . . . . 128

5.4.3 Combined Constraint Solving and Model Counting Performance 128

5.5 Comparison of Accuracy vs Performance . . . . . . . . . . . . . . 129

$5.6 \quad$ Evaluation Environment........................... 130

6 Results and Conclusions ...................... 131

6.1 Results .................................. 131

$6.1 .1 \quad$ Accuracy ................................ 132 
6.1.2 Performance ................................ 144

6.1.3 Accuracy vs Performance Comparisons . . . . . . . . . . . . . . 146

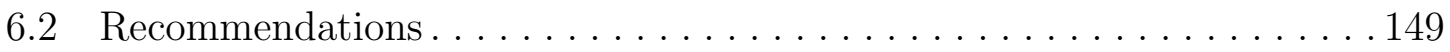

6.3 Threats to Evaluation Validity ......................... 151

6.3.1 Internal Threats to Validity . . . . . . . . . . . . . . . . . . 151

6.3.2 External Threats to Validity . . . . . . . . . . . . . . . . . 152

6.4 Conclusions.................................... 156

6.5 Possible Future Work . . . . . . . . . . . . . . . . . . . . . . . . 157

6.5.1 Additional testing . . . . . . . . . . . . . . . . . . 158

6.5.2 Enhancements . . . . . . . . . . . . . . . . . . . . 158

6.5 .3 Other . . . . . . . . . . . . . . . . . . . . . . . 160

$6.6 \quad$ Final Thoughts . . . . . . . . . . . . . . . . . . . . . . . . . 161

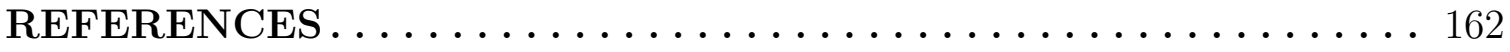




\section{LIST OF TABLES}

4.1 SCSF Third Party Dependencies . . . . . . . . . . . . . . . . 86

5.1 Data Columns Reported For Solved Constraints . . . . . . . . . . . . . . 110

5.2 New Data Columns Extracted from Constraint Sequence Values . . . . . 111

5.3 The Independent Variables in the Evaluation . . . . . . . . . . . . . . 113

5.4 The Number of Distinct Argument Combinations for String Operations 122

5.5 Accuracy vs Performance Comparison Analyses . . . . . . . . . . . . . . . . 129

6.1 Frequency of Branch Selection Agreement . . . . . . . . . . . . . . . . 132

6.2 Frequency of Branch Selection Agreement For Different Initial String Characteristics . . . . . . . . . . . . . . . . . . . . 133

6.3 Frequency of Branch Selection Agreement For Different Operations . . . 135

6.4 Frequency of Branch Selection Agreement For contains and equals Predicates . . . . . . . . . . . . . . . . . . . . . . . . 136

6.5 Model Counting Percentage Difference . . . . . . . . . . . . . . . . . . 138

6.6 Model Counting Percentage Difference For true and false Branches . . 138

6.7 Model Counting Percentage Difference For Different Initial String Char-

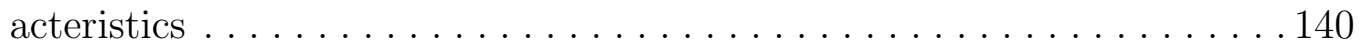

6.8 Model Counting Percentage Difference For Different String Operations . 141

6.9 Model Counting Percentage Difference For contains and equals Pred-

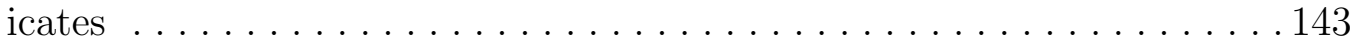


6.10 Solver Recommendations Based on Suitability . . . . . . . . . . . . . . . . 149 


\section{LIST OF FIGURES}

1.1 Password Matching Example . . . . . . . . . . . . . . . . . . 2

2.1 The testInt Java method demonstrating SE. . . . . . . . . . . 9

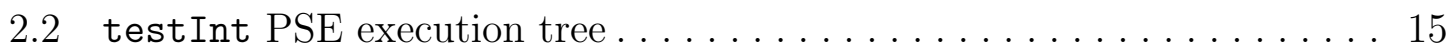

2.3 The testDiv Java method demonstrating the division problem in PSE. 18

2.4 The testStr Java method demonstrating PSE with string variables. . . 24

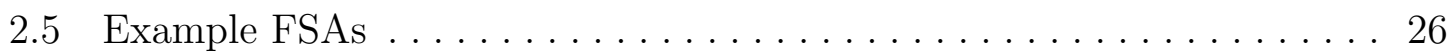

2.6 Initial Symbolic String Characteristics . . . . . . . . . . . . . . . . . 29

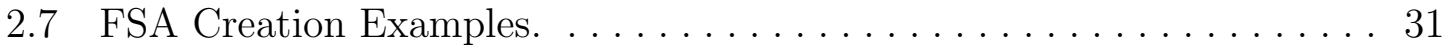

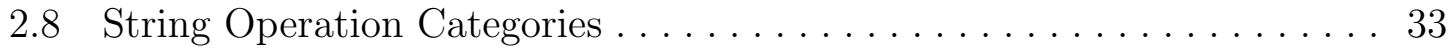

$2.9 \operatorname{FSA} \operatorname{Substring}(\mathcal{A}, 1,2)$ operation example $\ldots \ldots \ldots \ldots \ldots \ldots$

2.10 Two Substring operation algorithms . . . . . . . . . . . . 38

2.11 String Predicate Categories . . . . . . . . . . . . . . . . . 40

2.12 FSA Contains $\left(\mathcal{A}_{1}, \mathrm{~A}\right)$ true predicate example............ 43

2.13 An sample Java method that uses PSE with String variables. . . . . . . 45

3.1 Unbounded Automata Model: $\Sigma=\{\mathrm{A}, \mathrm{B}\}, k=2 \ldots \ldots \ldots \ldots$

3.2 Model counting algorithms for unbounded automata models . . . . . . 48

3.3 Concatenation $\left(\mathcal{M}_{1}, \mathcal{M}_{2}\right)$ example for unbounded automata model . . 50

$3.4 \operatorname{DeLETE}\left(\mathcal{M}_{1}, 1,2\right)$ example for unbounded automata model . . . . . . . 53

3.5 $\operatorname{ReplacE}\left(\mathcal{M}_{1}, \mathrm{~A}, \mathrm{~B}\right)$ example for unbounded automata model. . . . . . 54 
3.6 Bounded Automata Model: $\Sigma=\{\mathrm{A}, \mathrm{B}\}, k=2 \ldots \ldots \ldots \ldots$

3.7 Creation algorithm for bounded automata model . . . . . . . . . . . 55

3.8 Model counting algorithms for bounded automata models . . . . . . . . 57

3.9 Conchtenation $\left(\mathcal{M}_{1}, \mathcal{M}_{2}\right)$ example for bounded automata model . . . 57

$3.10 \operatorname{DELETE}\left(\mathcal{M}_{1}, 1,2\right)$ example for bounded automata model . . . . . . . . 59

$3.11 \operatorname{Replace}\left(\mathcal{M}_{1}, \mathrm{~A}, \mathrm{~B}\right)$ example for bounded automata model . . . . . . . 59

3.12 Aggregate Bounded Automata Model: $\Sigma=\{\mathrm{A}, \mathrm{B}\}, k=2 \ldots \ldots \ldots$

3.13 Algorithms to create FSA sequence for aggregate automata model . . . 61

3.14 Aggregate automata model utility algorithms for string operations and

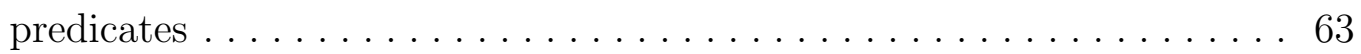

3.15 Coordinating algorithm aggregate automata models . . . . . . . . 66

3.16 Concatenation $\left(\mathcal{M}_{1}, \mathcal{M}_{2}\right)$ example for aggregate automata model . . 67

$3.17 \operatorname{DeLETE}\left(\mathcal{M}_{1}, 1,2\right)$ example for aggregate automata model . . . . . . . 69

$3.18 \operatorname{Replace}\left(\mathcal{M}_{1}, \mathrm{~A}, \mathrm{~B}\right)$ example for aggregate automata model . . . . . . 71

3.19 Weighted-Transition Aggregate Bounded Automata Model . . . . . . . . 72

3.20 Algorithms to create WFSA sequence for weighted automata model . . 75

3.21 Model counting algorithms for weighted automata models . . . . . . . . 77

3.22 Weighted automata model utility algorithms for string operations and predicates . . . . . . . . . . . . . . . . . . . . . . 79

3.23 Concatenation $\left(\mathcal{M}_{1}, \mathcal{M}_{2}\right)$ example for weighted automata model . . . 81

$3.24 \operatorname{DELEte}\left(\mathcal{M}_{1}, 1,2\right)$ example for weighted automata model . . . . . . . 82

$3.25 \operatorname{Replace}\left(\mathcal{M}_{1}, \mathrm{~A}, \mathrm{~B}\right)$ example for weighted automata model . . . . . . 83

4.1 The String Constraint Solver Framework (SCSF) . . . . . . . . . . 85

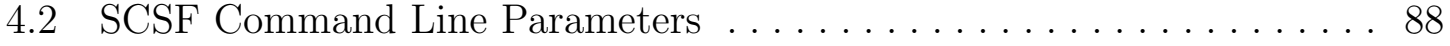




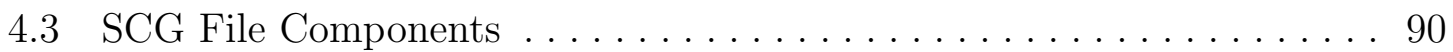

4.4 Synthetic SCG Generation Script Options . . . . . . . . . . . . . 92

5.1 The Evaluation Procedure . . . . . . . . . . . . . . . . . . 108

5.2 Literal Input String Type Graph Representation ............... 115

5.3 Simple Input String Type Graph Representation . . . . . . . . . . . . . 115

5.4 Complex Input String Type Graph Representation . . . . . . . . . . . 116

5.5 reverse Operation Graph Representation ................... 118

5.6 concat Operation Graph Representation ..................... 119

5.7 delete Operation Graph Representation . . . . . . . . . . . . . . 119

5.8 replace Operation Graph Representation . . . . . . . . . . . . . 120

5.9 Predicate Graph Representation . . . . . . . . . . . . . . . . . . 121

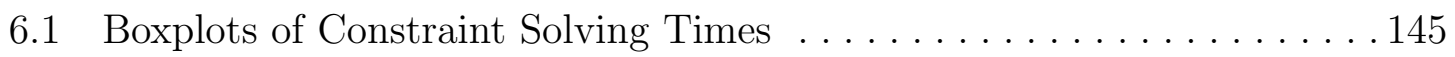

6.2 Boxplots of Model Counting Times and Constraint Solving Times . . . 146

6.3 Boxplots of Combined Model Counting and Constraint Solving Times . 147

6.4 Scatterplot of Percentage Difference vs Constraint Solving Times . . . . 148

6.5 Scatterplot of Percentage Difference vs Model Counting Times ....... 149

6.6 Scatterplot of Percentage Difference vs Combined Model Counting and

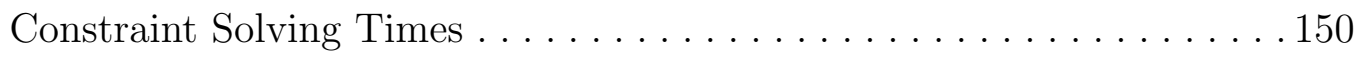




\section{LIST OF ABBREVIATIONS}

DFA - Deterministic Finite State Automaton

FSA - Finite State Automaton

MC - Model Count

NFA - Nondeterministic Finite State Automaton

PC - Path Condition

PSE - Probabilistic Symbolic Execution

SAT - Satisfiable

SCG - String Constraint Graph

SCSF - String Constraint Solver Framework

SE - Symbolic Execution

UNSAT - Unsatisfiable

WFSA - Weighted-Transition Finite State Automaton 


\section{LIST OF SYMBOLS}

$\mathcal{A} \quad$ Finite State Automata

$\mathcal{L}(\mathcal{A})$ Language of Finite State Automaton

$\mathcal{M}$ Automata-based Symbolic String Model

$\mathcal{S} \quad$ Solution Set

$\mathcal{W}$ Weighted-transition Finite State Automaton 


\section{CHAPTER 1}

\section{INTRODUCTION}

\subsection{Quantitative String Analysis}

Quantitative string analysis is a type of software engineering technique which quantifies the behaviour of a software program using the number of possible values a string variable can have along different execution paths in the program. Quantitative string analyses have many different applications in the software engineering and development processes including security vulnerability detection [17,37-41], bug and error detection [7], and test case generation [17]. The ability to analyze the behaviour of programs which quantitative string analysis allows is important to common string heavy programs such as web applications. Quantitative string analysis research has followed a few primary directions in recent years including quantitative information

flow analysis $[8,30,35]$ and probabilistic symbolic execution [1,27]. Despite these different approaches to quantitative string analysis, the different analysis techniques have a shared fundamental requirement: a constraint solver capable of quantifying the number of execution paths followed for a set of string values. Such a string constraint solver must rely on an underlying symbolic string model to count the number of string values which can occur along execution paths.

Quantitative string analysis has emerged as a research area in recent years due to the the ability to provide more information than the more common reaching analysis. 
A reaching analysis for string variables is used to determine if different sections of code in a program can execute for a given set of string values. Quantitative string analyses are used to count the number of executions which reach different portions of a program rather than the binary true or false result of the reaching analysis. The more robust quantitative data allows additional software engineering applications such as detection of program errors which can not be detected with a reaching analysis.

\subsection{Example}

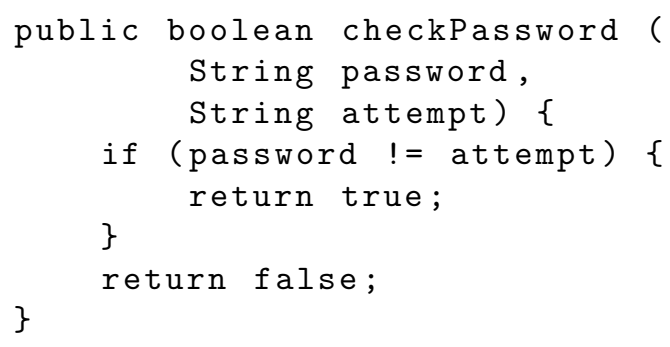

(a) Password checking function

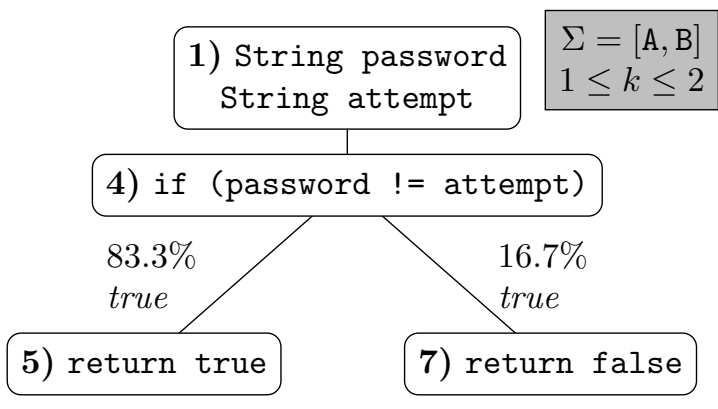

(b) checkPassword execution tree

Figure 1.1: Password Matching Example

Figure 1.1 demonstrates the usefulness of a quantitative string analysis when compared to a reaching analysis. The Java function in Figure 1.1a is used to check a password from user input (attempt) against an already known password (password). The function contains an error in line 4 where password and attempt are compared for inequality instead of equality as indicated from the subsequent returns of true and false on lines 5 and 7 respectively. Figure $1.1 \mathrm{~b}$ is the execution tree for this function which illustrates the code paths that can occur during execution of this function. For this example, the string alphabet $(\Sigma)$ has been restricted to only the letters A and B and the initial string length $(k)$ is either 1 or 2. As a result of these 
restrictions, the possible initial string values for both the password and attempt variables are the following 6 values: A, B, AA, AB, BA, and BB. The differences between the quantitative and reaching analyses is shown when evaluating line 4 of the program and processing the predicate: password != attempt. A reaching analysis shows that at least one of the six values for password is not equal to one of the six values for attempt as shown by the true on the branch from node 4 to node 5 in the execution tree. The reaching analysis also shows the same for the branch from node 4 to node 7 with another true label. Since both branches reach lines 5 and 7 of the program, no error is detected since that is the expected behavior. The additional information provided in a quantitative analysis is able to expose the error in the function. When the set of six string values which represent attempt are tested for equality with the set representing password, a total of 36 possible combinations of string values are evaluated for inequality. This results in $83.3 \%$ of these combinations evaluating as not equal and there for following the branch to line 5 of the function. The other $16.7 \%$ of combinations evaluate as equal and follow the branch to line 7 . The behavior of the function as shown by accepting $83.3 \%$ of password attempts for the restricted string values is not the expected behavior of the function. Through this example, it is clear that the additional information provided by a quantitative analysis allows for additional useful applications when compared to reaching analyses.

\subsection{Symbolic String Models}

While the example in Figure 1.1 clearly demonstrates the usefulness of a quantitative string analysis, the reliability of the analysis depends upon the accuracy of the number of execution paths computed from the set of possible string values. 
Because of an inability to store the set of all possible values for non-trivial string alphabets and string lengths, quantitative string analyses must make use of some form of symbolic string model to represent this set of values a string variable can have along different code paths in a program. Because string values in a program are often altered by string operations, the quantitative string analysis must also emulate these operations for the symbolic string model. For example, the Concatenate operation joins a second string to a first string so that the characters from the first string precede the characters from the second in the new string resulting from the operation, e.g. Concatenate $(\mathrm{A}, \mathrm{B})=\mathrm{AB}$. Similar to simulating string operations, string predicates must also be emulated for the symbolic string model in an analysis. For example, the EQUALS predicate checks if a first string value is equal to a second string value and returns true or false depending on if the strings are the save value, e.g. $\operatorname{Equals}(\mathrm{AB}, \mathrm{BA})=$ false. Due to these requirements for a symbolic string model, only a few symbolic string models have proven to be robust enough for use in string analysis research. One proven symbolic string model is the Finite State Automaton (FSA) which was chosen to perform this research analysis on string constraints.

\subsection{Thesis Statement}

This thesis analyzes four string constraint solvers which utilize finite state automata to model symbolic string values in the context of probabilistic symbolic execution. In particular this thesis addresses the following research statements:

- Accuracy:

- How often do automata-based solvers produce invalid analyses? 
- How does an automata-based string constraint solver solver compare in its calculation of branch probability to the actual branch probability? How do automata-based solvers compare in this calculation to one another?

- What characteristics of a string constraint effect accuracy of an automatabased solver? How large is this effect?

- Performance:

- What is the relative performance of one automata-based solver compared to others?

- How does the performance of different automata-based solvers compare for constraint solving specifically?

- How does the performance of different automata-based solvers compare for model counting specifically?

- How do the characteristics of a string constraint effect the performance of an automata-based solver?

- Accuracy vs. Performance trade-offs:

- What is the relationship between accuracy and performance for an automatabased solver?

- What automata-based solver produces the best combination of accuracy and performance?

- How is the relationship between accuracy and performance affected by specific characteristics of a string constraint?

\subsection{Contributions}

The main contributions of this thesis are as follows: 
- Identification of Collapse Problem (Sections 2.2.3, 2.3.2, 3.1.3, 3.2.3, 3.3.4, and $3.4 .6)$

- Four Automata-based Symbolic String Models (Sections 3.1, 3.2, 3.3, and 3.4)

- String Constraint Solver Framework (Chapter 4)

- String Constraint Model Counting Oracle (Sections 4.4.3 and 5.3.1)

- Evaluation of Suitability of Automata-based Symbolic String Models (Chapters 5 and 6$)$ 


\section{CHAPTER 2}

\section{BACKGROUND}

This chapter provides the background information relevant to this thesis. Section 2.1 explains Symbolic Execution (SE). Section 2.2 describes an enhancement of SE, Probabilistic Symbolic Execution (PSE). Section 2.3 explores the modeling of string constraints using symbolic string models. Finally, Section 2.4 details the modeling of string constraints using Finite State Automata (FSA) specifically.

\subsection{Symbolic Execution}

Symbolic Execution (SE) [24] is a path-sensitive static program analysis technique that determines what program inputs result in execution of different code paths. SE is an important software analysis technique with many useful applications such as detecting security vulnerabilities [26], detecting program errors [9], and aiding in generating test cases [22]. While SE was initially used with simple integer variable types, more recent research extends SE to analyze complex types such as floating point numbers [5], sets [10,25], and strings [34].

\subsubsection{Description}

When SE interprets a program, instead of executing the program on a set of concrete inputs values, SE uses a symbolic value each time the program requires 
a new input value. This means that SE maps each input variable $x$ in the program domain to a corresponding symbolic value $X$ in the symbolic codomain maintained by SE. Initially symbolic values represent all concrete values in the domain of that data type. For example an integer input variable $x$ is mapped to the symbolic value $X$ and has a corresponding solution set $S_{X}$ containing all possible integers, i.e. $m(x)=X$ and $S_{X}=\mathbb{Z}$. SE also maintains a symbolic program state, which is a mapping of a program variable to a corresponding symbolic expression. A symbolic expression is a finite combination of symbolic values and domain operations specific to the domain of the symbolic values. In SE, a symbolic expression is produced from a domain operation performed on one or more previous symbolic expressions and is usually assigned to a new program variable. For example, when processing the statement $y=x-2$ where $x$ is an input variable, SE adds the variable $y$ to the set of known variables with the symbolic expression $X-2$ where $X$ is a previous symbolic value where $m(x)=X$.

When SE encounters a conditional statement or branch condition, SE must follow either the true or false branch to continue the analysis. In order to explore each branch, SE generates a branch constraint, a symbolic predicate that restricts the set of concrete program inputs that can follow the branch path. The true branch constraint is generated by substituting the program variables in the branch condition for their corresponding symbolic expressions from the symbolic program state. The false branch constraint is generated from negating that branch condition, i.e., negating the true branch constraint.

Once a branch constraint is generated, it is conjoined with constraints of all previously taken branches to yield a single combined constraint called the path condition $(P C)$. If no previous branches exist, the $P C$ is true. $\mathrm{A} P C$ is a conjunction of 


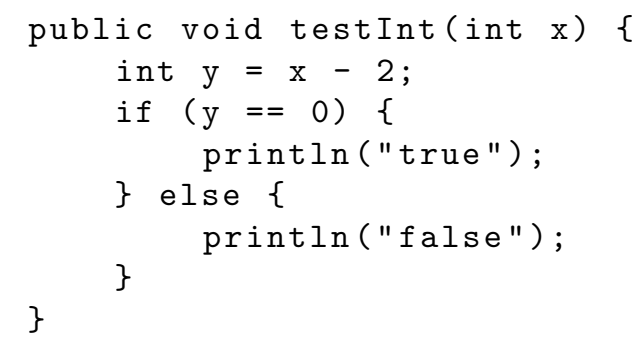

(a) testInt method

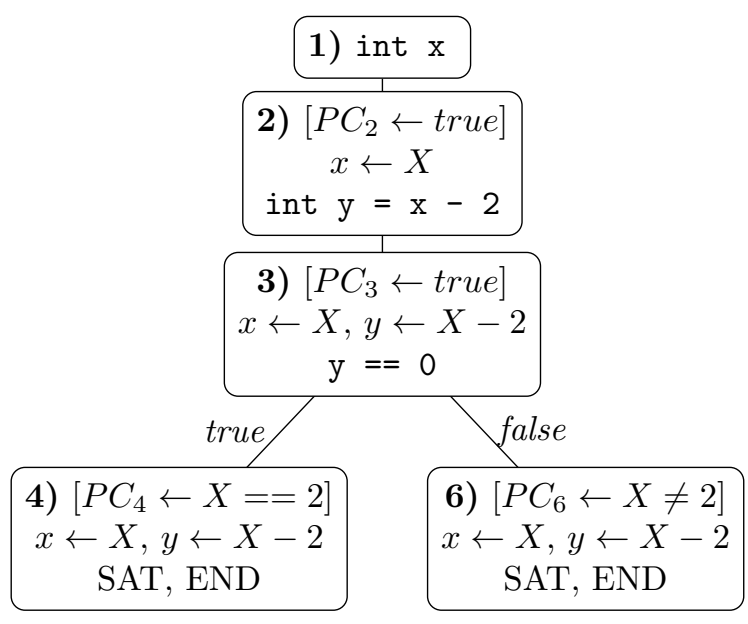

(b) testInt SE execution tree

Figure 2.1: The testInt Java method demonstrating SE.

predicates which restrict $S_{X}$ by a function $P C_{n}$ such that $P C_{n}(X) \rightarrow S_{n X}$, where $S_{n X}$ is the set of all concrete values for input variable $x$ which reach program point $n$. For example, if the $P C$ at a program point $n$ is $X==2$, the function $P C_{n}(X)$ would produce the set $\{2\}$ since only the integer 2 satisfies the predicate $X==2$. From this definition it is clear that a $P C$ exists for all statements in a program analyzed using SE starting with the initial $P C$ with a simple true predicate.

A typical SE analysis passes the $P C$ computed for a branch to a constraint solver to determine if the $P C$ of the branch is satisfiable (SAT) or unsatisfiable (UNSAT). The solver produces a symbolic model from the constraint and uses this model to determine satisfiability. If the solver determines a branch is SAT, SE continues interpreting statements along that code path. If the solver determines a branch is UNSAT, it is reported but not followed for any further analysis. In this way SE systematically analyzes all feasible paths of execution in a program. 


\subsubsection{Example}

A simple example of SE is shown in Figure 2.1 for the Java method test Int in Figure 2.1a. The SE tree in Figure 2.1b contains four nodes labeled 2, 3, 4, and 6 which correspond to the those line numbers in testInt. The $P C$ and variables for each node in the tree correspond to values before that line of code and after the previous line. The first step of SE is initializing symbolic variables for each input variable. The only input variable of the method is the integer parameter $x$ which is initialized to the unrestricted symbolic variable $X$. The $P C$ is also initialized at this time as $P C_{1} \leftarrow$ true. Both the initial $P C$ and the initial symbolic variable $x$ are displayed in node 2 as $P C_{2}$ and $x_{2}$ respectively. Next, the assignment statement on line 2 is interpreted and the new variable $y$ is initialized to the symbolic value $X-2$ from $x-2$ where $x$ is replaced by its symbolic variable $X$. This addition of the $y$ variable is reflected in node 3 of the SE tree representing the state before interpreting line 3.

Line 3 of testInt contains the first conditional statement of the method and requires the generation of both true and false branch constraints. To generate these constraints, the condition must be transformed into its symbolic equivalent by substituting for the variables in the conditional statement: $y==0 \rightarrow X-2==$ $0 \rightarrow X==2$. The true branch becomes $X==2$. Next, SE follows the true branch and must generate the new $P C$ for this code path by conjoining the true branch constraint, $X==2$, with $P C_{3}$ which yields $P C_{4} \leftarrow$ true $\wedge X==2$ which can be simplified to $P C_{4} \leftarrow X==2$. SE then determines that $P C_{4}$ is satisfiable since $2 \in S_{X}$, thus satisfying the $P C \leftarrow X==2$. This satisfied branch is reported by SE as indicated in the SE tree for node 4 by the "SAT" token. Since the true 
branch leads to line 4, SE next interprets this line which consists of a side-effect free statement, i.e., it does not alter the symbolic program state. After line 4, there are no more statements for SE to interpret along this code path within the testInt method. When SE encounters the end of a code path, it reports the end, as is shown in node 4 of the tree by the "END" token, and backtracks to explore other paths.

The false branch constraint for the condition on line 3 is generated by negating the true branch constraint: $\neg(X==2) \rightarrow X \neq 2$. SE must then generate the $P C$ for this false branch path by conjoining the branch constraint, $X \neq 2$, and $P C_{3}$ which produces $P C_{6} \leftarrow$ true $\wedge X \neq 2$ or $P C_{6} \leftarrow X \neq 2$. SE then determines that $P C_{6}$ is satisfiable since all but one integer in $S_{X}$ satisfies the condition ( 2 being the single exception). SE reports that this branch constraint is satisfied as shown in node 6 of the tree by the "SAT" token. Following the false branch SE advances to line 6 along the current code path. Since the statement on line 6 is side-effect free, SE attempts to advance to the next statement. Because there are no more statements along this path, SE produces the "END" token. At this point SE has exhaustively explored all possible code paths within the testInt method and has found that both branches of the condition in line 3 are SAT. This information reported by SE can be used for many applications such as in a reachability analysis which can conclude that lines 4 and 6 are reachable due to the true and false branches being SAT.

\subsubsection{Limitations}

The largest drawback of SE is poor scalability due to the path sensitive nature of the technique requiring the traversal of too many paths. The number of paths grows exponentially as the number of conditional statements in the program increases. Additionally, the presence of loops in the program increases the performance cost since 
new branch constraints are generated each time the loop condition is checked. To mitigate this problem, SE exposes only a finite number of loop iterations which limits the performance cost at the expense of analysis completeness. Trade-offs of this type between performance and accuracy are commonly used to adjust the performance of SE based analyses, allowing otherwise impractical analyses to be completed.

SE is also limited by the quality of information it reports, where satisfiability of a code path provides only a coarse 1 or 0 approximation of the probability that the path will be executed. This lack of quantitative information limits the types of analyses which can be conducted using the SE technique. This limitation can be seen in the testInt example in Figure 2.1 where the condition on line 3 produced a true branch with one satisfying input, 2 , while the false branch is satisfied by all the remaining inputs. Clearly the false branch is overwhelmingly more probable to execute than the true branch, but SE simply reports both as SAT.

Both the performance and the quantitative information limitation are addressed by the probabilistic symbolic execution technique.

\subsection{Probabilistic Symbolic Execution}

Probabilistic Symbolic Execution (PSE) [16] is an enhancement of SE which provides a quantitative analysis of execution for a software program in the form of conditional branch probabilities rather than branch satisfiability (SAT or UNSAT) reported by SE. This enhancement allows PSE to prioritize code paths more likely to be executed while differing less probable paths to analyze when time permits. Though PSE is an emerging static analysis technique, it is already being used to aid program understanding and error detection [16], to compute software reliability [4,13], and 
to provide quantitative information flow analysis for software security [29]. While most of this early research has been restricted to analyses of integer variables, there is increasing interest into using PSE to analyze string variables in programs [1].

\subsubsection{Description}

PSE updates the symbolic program state in exactly the same way as traditional SE, but handles reporting conditional branches differently. In SE the constraint solver reports a branch constraint as either SAT or UNSAT, which is in fact a coarse approximation of the probability of executing a branch where UNSAT and SAT correspond to probabilities 0 and 1 respectively. PSE aims to provide a more detailed understanding of program execution through the use of program path probabilities. These path probabilities allow for quantitative analyses of a program rather than satisfiability analyses.

When PSE processes a conditional branch, it determines which of two branch paths to follow by selecting the branch with the larger execution probability. Each execution probability for a branch is the ratio of the number of concrete execution paths which satisfy the branch condition to the number of concrete execution paths which reach the branch condition. This probability calculation is only possible due to the bijective relationship between the set of all execution paths and the set of input value combinations which is the $k$-fold Cartesian product produced from the sets of concrete values for $k$ input variables. Utilizing this relationship, the solver can calculate the number of execution paths reaching a program point as the model count of the solution set for the program point's $P C$.

Definition A solution set, denoted as $\mathcal{S}$, is the $k$-tuple set of solution values for a symbolic path constraint or $P C$. Alternatively, a solution set $\mathcal{S}_{n}$ is the $k$-fold Cartesian 
product of the sets of values for $k$ input variables which can reach at a program point $n$, i.e. $\mathcal{S}_{n}=S_{n X 1} \times \cdots \times S_{n X k}$ where $S_{n X i} \forall i: 1 \leq i \leq k$.

Definition Model count, denoted as MC, is the total number of solutions for a symbolic constraint or $P C$. Alternatively, model count is the cardinality of a solution set, i.e. $M C\left(P C_{n}\right)=\left|\mathcal{S}_{n}\right|$ at a program point $n$.

The branch probability calculation $P_{b}$ is shown in Formula 2.1 as the ratio of the $\mathrm{MC}$ of the $P C$ after the branch $M C\left(P C_{b}\right)$ to the $\mathrm{MC}$ of the $P C$ before the conditional statement is interpreted $M C\left(P C_{c}\right)$.

$$
P_{b}\left(P C_{b}, P C_{c}\right)=\frac{M C\left(P C_{b}\right)}{M C\left(P C_{c}\right)}
$$

In addition to branch probability, the overall probability that a program point is reached can also be used by PSE. This overall probability, called global execution probability and denoted as $P_{g}$, can be used to prioritize all the remaining unexplored paths for analysis. It is the ratio of the number of concrete execution paths reaching a program point to the total number of concrete execution paths in the program. Formula 2.2 shows this probability calculation as the ratio of the $\mathrm{MC}$ of the $P C$ at program point $n\left(M C\left(P C_{n}\right)\right)$ to the $\mathrm{MC}$ of the initial program inputs $\left(M C\left(P C_{\text {init }}\right)\right)$.

$$
P_{g}\left(P C_{n}, P C_{i n i t}\right)=\frac{M C\left(P C_{n}\right)}{M C\left(P C_{i n i t}\right)}
$$




\subsubsection{Example}

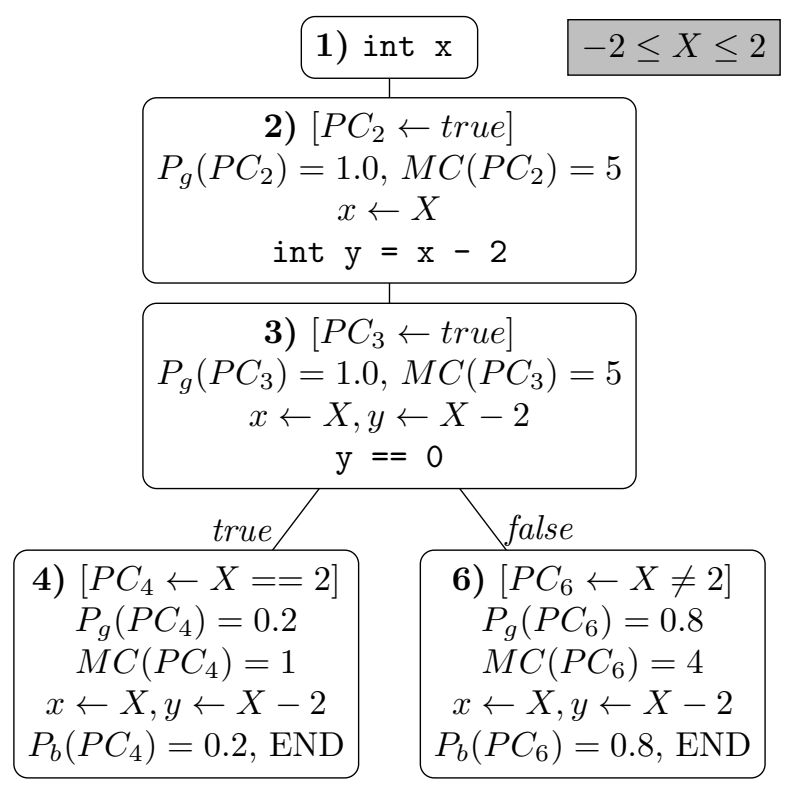

Figure 2.2: testInt PSE execution tree

To demonstrate PSE, consider the testInt Java method from Figure 2.1a which produces the PSE tree in Figure 2.2. For this PSE example, the input variable domain is restricted to $\{i \in \mathbb{Z} \mid-2 \leq i \leq 2\}$ in order to better demonstrate PSE. This is shown as the grey area left of the PSE tree. This restriction results in $S_{X}=\{-2,-1,0,1,2\}$ for symbolic variable $X$ initialized from input variable $x$. PSE then interprets the testInt method identical to SE until line 3 where the first conditional statement is encountered. The program variables before the execution of line 3 have symbolic values $x=X$ and $y=X-2$ just as in SE. PSE interprets the condition statements exactly as SE by substituting variables for the corresponding symbolic values. This produces $P C_{4} \leftarrow X==2$ for the true branch and $P C_{6} \leftarrow X \neq 2$ for the false branch.

Instead of determining satisfiability for each branch, PSE calculates the execution probability of each branch using Formula 2.1. The true branch probability is 
calculated using Formula 2.1 as $P_{b}\left(P C_{4}, P C_{3}\right)$ where the solution sets for $P C_{4}$ and $P C_{3}$ are $\mathcal{S}_{4}=\{2\}$ and $\mathcal{S}_{3}=\{-2,-1,0,1,2\}$ respectively. The resulting true branch probability is 0.2 . The false branch probability is calculated in the same way again using Formula 2.1 as $P_{b}\left(P C_{6}, P C_{3}\right)$ where the solution sets for $P C_{6}$ and $P C_{3}$, which are $\mathcal{S}_{6}=\{-2,-1,0,1\}$ and $\mathcal{S}_{3}=\{-2,-1,0,1,2\}$ respectively. The resulting false branch probability is 0.8 .

With both branch probabilities calculated, PSE chooses to explore the false branch since it is more likely to be executed than the true branch. Upon reaching the end of the path at line 6, PSE reports an "END" token. PSE next backtracks to the single remaining path to explore, following the true branch from the branch condition on line 3 to line 4. PSE reports with another "END" token after line 4 and completes the analysis since no unexplored paths remain. This analysis determines that line 6 is four times more likely to be executed than line 4 under the restricted input domain.

\subsubsection{Limitations}

While PSE uses probabilities for different code paths to address the performance cost of SE, it does incur a new performance cost. These additional computations are required for the MC used to compute branch probabilities. The performance cost of the MC calculation depends on the algorithm used to calculate the MC for each branch. The MC calculation algorithm performance cost is specific to each underlying model used to represent the symbolic values. In this way, the choice of symbolic model affects the performance of a PSE analysis. Just as in SE, PSE can sacrifice accuracy for better performance by over-approximating solution sets. However, unlike regular SE which identifies branches as SAT or UNSAT, PSE determines the likelihood of taking that branch. Such calculations are more sensitive to a loss in accuracy. 
In addition to performance issues, PSE also suffers from accuracy problems which can affect the validity of the analysis. A PSE analysis is invalid if it calculates that a path $P C_{x}$ has a higher probability than another path $P C_{y}$, i.e., $P_{P S E}\left(P C_{x}\right)>$ $P_{P S E}\left(P C_{y}\right)$, while the opposite is actually true, i.e. $P_{a c t}\left(P C_{x}\right)<P_{a c t}\left(P C_{y}\right)$. We have used the term collapse to describe the problem which results in this type of invalid analysis. A collapse occurs when two or more distinct possible values in the solution set for a variable constraint before an operation or predicate are "collapsed" into a single possible value in the solution set of the variable constraint as a result of the operation or predicate. This collapse results from the combination of two factors: non-injective variable operations and the assignment of operation results to new symbolic values.

An injective function is a function which never maps distinct elements from its domain to the same element of its codomain. In the context of a program, a non-injective function maps two or more combinations of parameters to the same concrete value from the operation. However, a non-injective operation alone is not sufficient to produce an invalid analysis. For example, integer variables are able to use non-injective operations such as division but still do not suffer from the collapse problem because integer variables can be expressed as functions of symbolic variables. These functional relationships between integer variables define $n$-dimensional convex polytopes which contain the solution set integers within.

The second factor causing collapse problems is the assignment of operation results to new symbolic variables. That is the creation of a new symbolic variable as the result of an operation with no explicit relation to the symbolic value arguments of the operation. For example, the symbolic value $Y$ is assigned to the integer variable $y$ from the integer division operation where the symbolic value $X$ restricted by the 


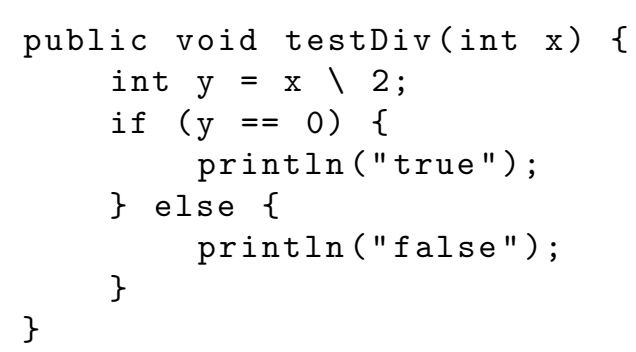

(a) testDiv method

\begin{tabular}{|c|c|c|c|c|}
\hline$P C_{n}$ & $\mathcal{S}_{n}$ & $\left|\mathcal{S}_{n}\right|$ & $V_{n}$ & $\left|V_{n}\right|$ \\
\hline$P C_{2}$ & $\{-2,-1,0,1,2\}$ & 5 & $\{-2,-1,0,1,2\}$ & 5 \\
\hline$P C_{3}$ & $\{-2,-1,0,1,2\}$ & 5 & $\{-2,-1,0,1,2\}$ & 5 \\
\hline$P C_{4}$ & $\{0\}$ & 1 & $\{0,0,0\}$ & 3 \\
\hline$P C_{6}$ & $\{-1,1\}$ & 2 & $\{-1,1\}$ & 2 \\
\hline
\end{tabular}

(b) Actual Solution Set vs.

Approximated Solution Set

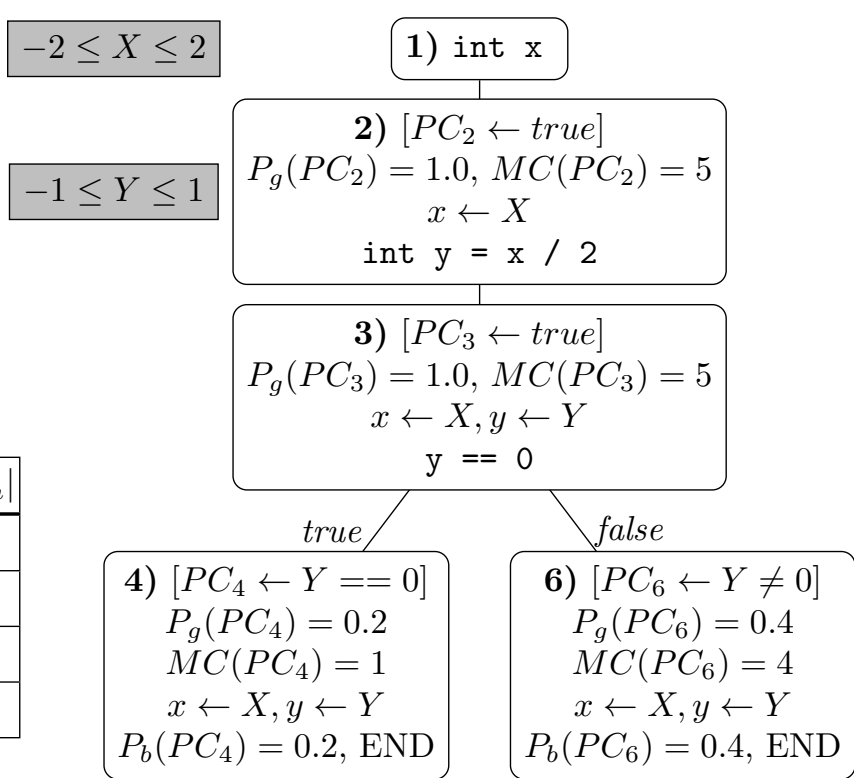

(c) testDiv PSE execution tree

Figure 2.3: The testDiv Java method demonstrating the division problem in PSE

inequality $-2 \leq X \leq 2$ is divided by the integer 2, i.e., $y \leftarrow Y: y=\frac{X}{2}$. This assignment requires the set of values represented by $Y$ to contain each of the values represented by $X$ divided by 2, resulting in a restriction of $Y$ by the inequality $-1 \leq Y \leq 1$. Assignments in this manner usually occurs due to a complex variable domain and requires advanced models to accurately model the symbolic values for the domain. This results in a need to produce a new symbolic value from variable operations. The combination of non-injective variable operations and producing new symbolic values results in a symbolic value which cannot have a one-to-one relation to the operation inputs and similarly cannot have a one-to-one relation to the execution paths of the program.

The example in Figure 2.3 demonstrates a collapse problem for integer variables. Since integer variables do not usually produce collapse problems, we assign the result of the division operation on line 2 to a new symbolic value, fulfilling the previously 
discussed requirements for the collapse problem to occur. The Java method testDiv in Figure 2.3a is a slight modification of the test Int method from Figure 2.1a where a division operation is used on line 2 instead of a subtraction operation. A table is presented in Figure 2.3b displaying for each line $n$ in the program: the solution set denoted $\mathcal{S}_{n}$, the size of the solution set denoted $\left|\mathcal{S}_{n}\right|$, the bag (multiset) of actual values which would appear in the program denoted $V_{n}$, and the number of values in the bag denoted $\left|V_{n}\right|$. The PSE execution tree is presented in Figure 2.3c. As in the previous example, the integer variable domain for the input values is restricted to the set of integers values $\{-2,-1,0,1,2\}$. The results of the assignment of $y$ on line 2 are shown in the tree where $y \leftarrow Y$ instead of assigning $y$ in relation to $X$ as was done in testInt in Figure 2.2. The bag of actual values and the solution sets are in agreement for both $P_{2}$ and $P C_{3}$ due to both being true, this also results in a $\mathrm{MC}$ of 5 representing all the execution paths in the program.

The collapsed value problem emerges when the true and false branch probabilities are calculated from the branch constraints. The true branch is followed when the input $(y)$ value is 0 which occurs for three different $x$ input values $\{-1,0,1\}$ and therefore three execution paths. However, PSE calculates the $M C\left(P C_{4}\right)$ from $\mathcal{S}_{4}$ which is $\{0\}$ since 0 is the only value which satisfies the $P C_{4}$. This is a clear example of the collapse of multiple execution paths represented by the $x$ inputs $-1,0$, and 1 into the single value 0 in the solution set for $P C_{4}$. The subsequent probability calculations are affected by this difference in $\mathrm{MC}$ where the branch probability of 0.2 is obtained using Formula 2.1. This is in contrast to the bag of actual values in Table $2.3 \mathrm{~b}$ which shows 3 out of 5 execution paths execute line 4 for an actual probability of 0.6. The false branch is modeled correctly for PSE where $P_{b}\left(P C_{6} / P C_{3}\right)=0.4$ corresponds to the 2 of 5 execution paths execute line 6 . This difference in the 
probability of the true branch without a difference in the probability of the false branch causes PSE to determine that the false branch is more likely to be executed since $P_{b}\left(P C_{6} / P C_{3}\right)>P_{b}\left(P C_{4} / P C_{3}\right)$. The value table clearly shows that with 3 of 5 execution paths, the true branch is actually more likely than the false resulting in an invalid analysis reported by PSE.

This collapse problem is an example of a limitation of PSE due to the dependence on the MC obtained for $P C$ s. Since the MC is calculated from the solution set of a constraint, the accuracy of PSE depends upon the accuracy of the symbolically modeled constraint. PSE analyses of integer variables avoids the collapse of paths by calculating a MC from the volume of the convex polytope which is defined by the constraint inequalities in a $k$-dimensional vector space [16] where $k$ is the number of input variables to the program. Other complex data types are not able to easily utilize a vector space to model constraints and must calculate MCs from the solution set of the a constraint, which results in the collapse of paths. This becomes a common problem when analyzing other variable data types such as strings where a nearly all operations are not injective. It is clear that the choice of underlying symbolic model is key to minimizing or eliminating the impact of this collapsing paths problem.

\subsection{Modeling String Constraints}

While many methods exist for modeling symbolic string variables, only a few of these types of representation have been found appropriate for string analysis research. These representations include most significantly bit-vectors [3,23], axiom based models [42], and finite state automata (FSA) [1,7,18,19,34,36-41]. These different string models have been used to detect SQL injection vulnerabilities [17,37-41], to detect 
string variable errors in programs [7], and to generate test cases for string variable inputs [17]. This thesis focuses on the use FSA to model symbolic strings.

\subsubsection{Symbolic String Models}

Strings form a fundamental datatype in general purpose programming languages. A string is a finite sequence of symbols or characters that are chosen from a nonempty set of symbols called an alphabet, denoted $\Sigma$. The length of a string, $k$, can be any non-negative integer with the special case of the 0 length string called the empty string $\varepsilon$. Strings are represented in many different ways depending on the programming language such as a null-terminated array in C, an explicit length array in Java, or a singly linked list in Haskell. Additionally, often different string-like data types can exist in the same programming language. For example, the String and StringBuilder classes in Java are different data types but both represent the same abstract string. This is similar to abstract integers implemented as both 32 and 64-bit concrete integer data types in a programming language. Because of the common abstraction for string-like data types, each can be modeled by the same symbolic string model just as different $n$-bit integers are modeled by the same integer inequalities. In addition to modeling each string-like data type through a single symbolic string model, the operations and predicates of the the string-like data type must be modeled as well.

Formal language theory defines a set of simple operations for strings under which the language is closed, e.g. ConcAtenate. Unlike integer arithmetic which is often included in the programming language itself, string functions and operations are usually implemented as library functions. For example, the Java StringBuilder class includes the append method which is an implementation of the abstract string 
operation Concatenate. While it is possible to analyze these functions as part of the detailed analysis, such an approach would be inefficient. Instead, string functions are interpreted as single operations for the abstract string data type allowing algorithms to model distinct operations for the chosen symbolic string model. While this approach is sufficient for most common string operations, the complexity of others, e.g. FORMAT has rendered this approach impractical for those string functions. Additionally, string operations which return data types other than strings are difficult to analyze. These operations are mixed constraint type operations where the analysis must utilize symbolic values for the other data types being returned from the operation. For example, the Java String class contains the method length which returns an integer. In PSE, a symbolic integer model would be needed to model the behavior of the result of the length operation and an algorithm would be needed to simulate the correct possible length values of the symbolic string model.

String predicates are handled similarly to mixed constraint type string operations since a function is applied to a string data type and returns a boolean data type. Unlike string operations which occur in a particular branch of execution in a program, most predicates occur as branching conditions. For example the Java String class contains the equals method which is an implementation of the abstract string predicate EQUALS and is usually used in if statements to determine if the code in the statement is executed. In PSE, this is represented in both branches of the predicate by transforming using appropriate algorithms such that the symbolic string model following the branch condition models the string values satisfying the branch condition. For example, the symbolic string variable $y$ represents the string variable y in the Java if condition y. contains ("B") where $\Sigma=\{\mathrm{A}, \mathrm{B}\}, k=1$, and $y=\{\varepsilon, \mathrm{A}, \mathrm{B}\}$ before the branch condition. After this condition, $y_{t}=\{B\}$ follows the true branch 
and represents the string values where $\operatorname{Contains}(y, \mathrm{~B})=$ true. $y_{f}=\{\varepsilon, A\}$ follows the false branch and represents the string values where $\operatorname{Contains}(y, \mathrm{~B})=$ false. Just as some string operations impractical to emulate for symbolic string models due to the operation complexity and/or the mixed constraint data types, some string predicates can occur with sufficient complexity to render an analysis of the predicate impractical.

Due to these requirements for symbolic string models, only a limited number of model types have been found to be sufficiently robust to represent the alphabet and length requirements while also being flexible enough models to simulate operations and predicates.

\subsubsection{Limitations}

While symbolic string models are currently used in many areas of string analysis research, some limitations still exist for all such models. One such limitation we have identified in our explorations of symbolic string models is a collapse problem which occurs as a result of some sequences of string operations and/or predicates. As shown in Section 2.2.3, non-injective operations can result in the "collapse" of multiple values in the constraint solution set (execution paths) into a single value in the resulting solution set. This is a common problem for string variables since many common string operations such as SUBSTRING and REPLACE are non-injective operations.

Figure 2.4 illustrates an example of the collapse problem for string variables. PSE of the Java method testStr in Figure 2.4a produces the corresponding tree in Figure 2.4c. Table $2.4 \mathrm{~b}$ presents a table that displays for each line $n$ in the program: the solution set denoted $\mathcal{S}_{n}$, the size of the solution set denoted $\left|\mathcal{S}_{n}\right|$, the bag (multiset) 


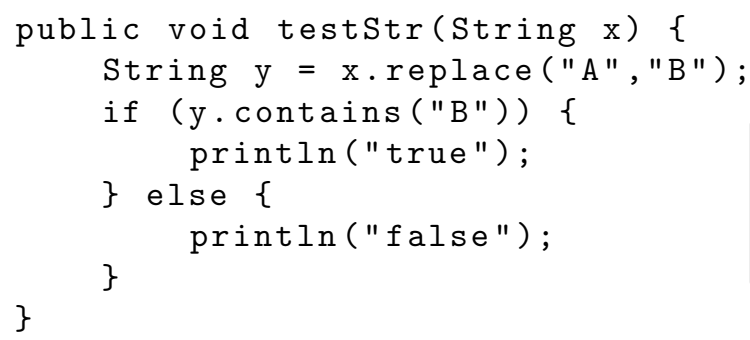

(a) testStr method

\begin{tabular}{|c|c|c|c|c|}
\hline$P C_{n}$ & $\mathcal{S}_{n}$ & $\left|\mathcal{S}_{n}\right|$ & $V_{n}$ & $\left|V_{n}\right|$ \\
\hline$P C_{2}$ & $\{\varepsilon, \mathrm{A}, \mathrm{B}\}$ & 3 & $\{\varepsilon, \mathrm{A}, \mathrm{B}\}$ & 3 \\
\hline$P C_{3}$ & $\{\varepsilon, \mathrm{A}, \mathrm{B}\}$ & 3 & $\{\varepsilon, \mathrm{A}, \mathrm{B}\}$ & 3 \\
\hline$P C_{4}$ & $\{\mathrm{~B}\}$ & 1 & $\{\mathrm{~B}, \mathrm{~B}\}$ & 2 \\
\hline$P C_{6}$ & $\{\varepsilon\}$ & 1 & $\{\varepsilon\}$ & 1 \\
\hline
\end{tabular}

(b) Actual Solution Set vs. Approximated Solution Set

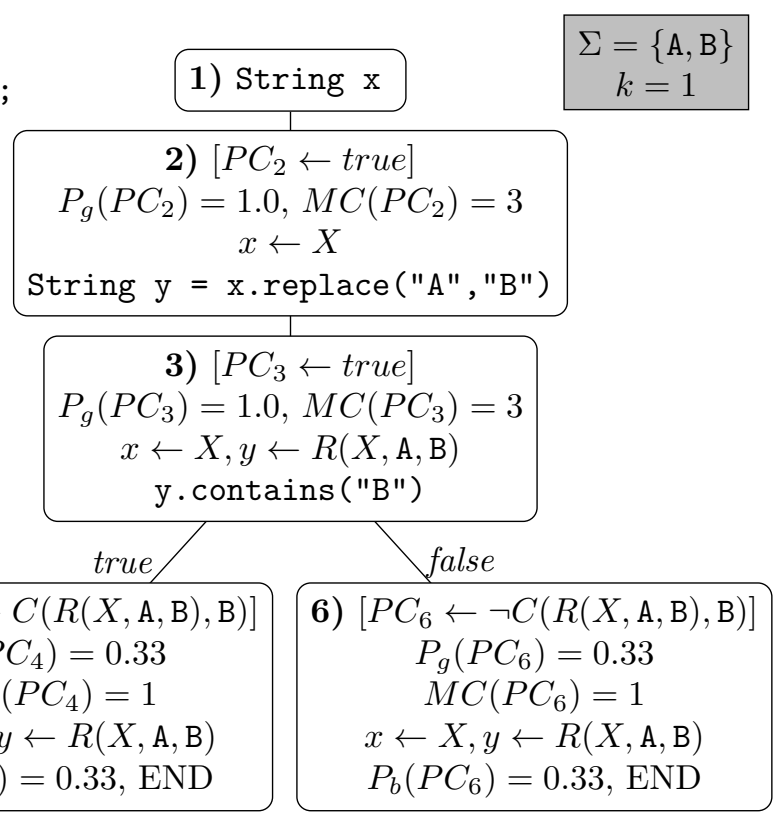

(c) testStr PSE execution tree

Figure 2.4: The testStr Java method demonstrating PSE with string variables.

of actual values which would appear in the program denoted $V_{n}$, and the number of values in the bag denoted $\left|V_{n}\right|$. The input strings for testStr is restricted to the alphabet $\Sigma=\{\mathrm{A}, \mathrm{B}\}$ and limited to an initial length $k=1$ in order to simplify the example. As a result of this restriction, the input string variable $x$ can only have three possible values: $\varepsilon$, A, or B. These initial input values assigned to the input variable $x$ as the symbolic string value $X$ as shown in node 2 of the PSE tree. The bag of actual values and the solution sets are in agreement for both $P C_{2}$ and $P C_{3}$ due to both being true, this also results in a MC of 5 representing all the execution paths in the program.

Just as in testDiv from Figure 2.3, the collapse problem emerges when calculating probabilities for the true and false branches of the conditional statement. The true branch will be followed when the $y$ string contains the B symbol which occurs for two 
different $x$ input strings $\{A, B\}$ and therefore two distinct execution paths. However, PSE calculates the $M C\left(P C_{4}\right)$ from $\mathcal{S}_{4}$ which is $\{\mathrm{B}\}$ since B is the only string value of $y$ which satisfies $P C_{4}$. It is clear that the two execution paths represented by the $x$ values A and B have collapsed into the single solution set value B. The subsequent branch probability calculation are affected by the difference in MC and produce a probability of 0.33 using Formula 2.1. The actual execution probability can be calculated from the actual bag of string values shown in the table for $P C_{4}$ where 2 out of 3 execution paths execute line 4 for an actual probability of 0.66 . The false branch of the condition is modeled correctly with a branch probability of 0.33 representing 1 out of 3 execution paths executing line 6. Due to the collapse problem, PSE will report that line 4 is equally likely to execute as line 6 when line 4 is actually twice as likely to execute as line 6 . This example clearly demonstrates how easily an invalid string analysis can occur with all commonly known symbolic string models.

\subsection{Modeling String Constraints with Finite State Automata}

All currently known symbolic string models suffer from the problem of noninjective path collapse. In order to guarantee a valid PSE analysis for string variables, a symbolic string model is needed which is not susceptible to this problem. Currently, no symbolic string model is known which avoids this problem, thus one must be created. While an entirely novel symbolic string model could be created to avoid this collapse problem while maintaining acceptable performance, it would be much easier to refine a known symbolic string model with proven performance in string analyses. As previously discussed, common model choices for symbolic strings include axiombased models, bit-vector based models, and finite state automaton based models. Of 
these choices, the automaton based model was chosen for modification in this work due to the flexible data structure and existing Java libraries which already support using automata-based models in SE.

\subsubsection{Finite State Automata}

A finite state automaton (FSA) is a mathematical model of computation that operates in response to an external sequence of symbols. A FSA accepts as input a string and either accepts or rejects the string as the result of processing this string. The set of strings accepted by a $\operatorname{FSA}(\mathcal{A})$ is called the language of the FSA, denoted as $\mathcal{L}(\mathcal{A})$, and is always a regular language. More formally, a deterministic finite state automaton $\mathcal{A}$ is defined as the quintuple $\left(Q, \Sigma, \delta, q_{0}, F\right)$ where

- $Q$ is a finite set of states, $Q \neq \varnothing$

- $\Sigma$ is a finite set of symbols called the alphabet, $\Sigma \neq \varnothing$

- $\delta$ is the transition function, $\delta: Q \times \Sigma \rightarrow Q$

- $q_{0}$ is the start state, $q_{0} \in Q$

- $F$ is the set of accepting or final states, $F \subseteq Q$

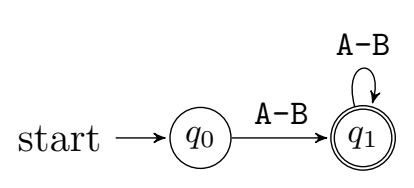

(a) An example Finite State Automaton

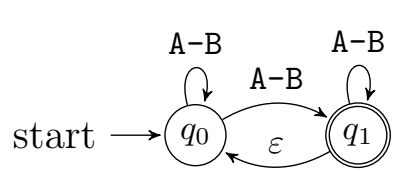

(b) An Non-Deterministic FSA

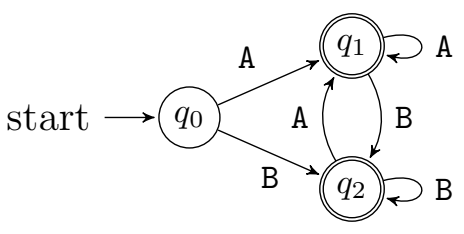

(c) A Non-Minimal FSA

Figure 2.5: Example FSAs

Figure 2.5a is a diagram of a simple FSA that will be used to explain and demonstrate the FSA quintuple. The FSA represented by the diagram accepts a 
non-empty input string of any combination of A and/or B symbols. The set of states $Q$ for this FSA contain only two states, $q_{0}$ and $q_{1}$, i.e. $Q=\left\{q_{0}, q_{1}\right\}$. The start state of the FSA is the $q_{0}$ state and is not an accepting state, i.e. $q_{0} \notin F$. The state $q_{1}$ is an accepting state, i.e. $q_{1} \in F$. The alphabet $\Sigma$ of this FSA consists of the two symbols A and B meaning that no other symbols can appear in a string accepted by the automaton. The transition function $\delta$ is comprised of the four distinct transitions: $\left(q_{0}, \mathrm{~A}\right) \rightarrow q_{1},\left(q_{0}, \mathrm{~B}\right) \rightarrow q_{1},\left(q_{1}, \mathrm{~A}\right) \rightarrow q_{1},\left(q_{1}, \mathrm{~B}\right) \rightarrow q_{1}$. In the diagram, the four distinct transitions are represented by only two directed lines: the line from $q_{0}$ to $q_{1}$ for the range of symbols from $\mathrm{A}$ to $\mathrm{B}$ and the line from $q_{1}$ to itself for the same range of symbols. This FSA begins its operation with the start state $q_{0}$ as the current state when processing the input string. If the input string is the empty string, the FSA has completed processing the input string and it is rejected since $q_{0} \notin F$ in this FSA. If the symbol is not $\mathrm{A}$ or $\mathrm{B}$, the input string is also rejected since there is no transition from state $q_{0}$ for any other symbols. When the FSA processes the first symbol and it is either $\mathrm{A}$ or $\mathrm{B}$, the current state is changed to state $q_{1}$ as required by the transition function $\delta$. Similarly to state $q_{0}$, when a A or B symbol is processed while state $q_{1}$ is the current state, $\delta$ requires updating the current state to $q_{1}$. Also similarly to state $q_{0}$, if a symbol other than A or B is processed while the current state is $q_{1}$, the input string is rejected. When the last symbol in the input string is processed and the string has not already been rejected for invalid symbols, if the current state is an accepting state like $q_{1}$, then the input string is accepted, otherwise the input string is rejected.

One characteristic of FSAs important for understanding the use of automata as symbolic string models is determinism where a FSA is either a deterministic (DFA) or non-deterministic (NFA) automaton. For DFAs, the codomain of the transition 
function $\delta$ is a set of states rather than a powerset of states. In other words, every state has only a single transition possible for every input symbol. Additionally, NFAs allow the use of epsilon transitions, a transition from one state to another without processing any input symbols: $\left(q_{\text {current }}, \varepsilon\right) \rightarrow q_{\text {new }}$. The FSA diagramed in Figure $2.5 \mathrm{~b}$ is a NFA which accepts the same language of strings as the previous FSA in Figure 2.5a which is a DFA. In the NFA diagram, the transition function from the state $q_{0}$ has two possible result states when processing either of the symbols A or B. The NFA also contains an epsilon transition from the state $q_{1}$ to the state $q_{0}$. One important property of DFAs and NFAs is the relationship between the two where all DFAs $\left(\mathcal{A}_{D}\right)$ are also NFAs $\left(\mathcal{A}_{D}\right)$ such that $\mathcal{A}_{D} \subset \mathcal{A}_{N}$. While NFAs have a different transition function $\delta$ than DFAs, every NFA has an equivalent DFA where both automata accept the same language of input strings. Because of this property, every NFA can be converted to an equivalent DFA using a method known as subset construction [31]. Due to these properties and methods, it is often easier to construct a NFA to accept a language of strings and then convert the automata to an equivalent DFA rather than attempting to construct the DFA instead.

A type of automaton which is important for understanding automata as symbolic string models is a minimal automaton. For each regular language, there exists a minimal DFA which accepts the language. This minimal DFA has no equivalent DFA with a smaller number of states. A minimal DFA can be created from any non-minimal DFA by removing unreachable automaton states and merging equivalent automaton states. Figure 2.5c shows a non-minimal DFA which accepts the same language as the minimal DFA in figure 2.5a. There are three well common algorithms used for minimizing DFAs: Hopcroft's algorithm [20], Moore's algorithm [28], and Brzozowski's algorithm [6]. Each of these three algorithms have different best case 
and average case run times which make the choice of algorithm to use in minimization variable depending upon the context in which it will be used.

\title{
2.4.2 Automata as Symbolic String Models
}

Automata can be used to model symbolic string values such that the language of the automaton is the solution set. There are four important areas of focus when modeling symbolic string constraints with automata: initializing the automata for the input string variable, emulating string operations, processing predicate branch conditions, and model counting the symbolic string automata.

\section{Initializing String Variables}

\author{
Alphabet: $\quad$ Set of symbols \\ Bounding Length: Integer upper bound \\ String Type: $\quad$ Null (Empty), Concrete (Empty String, Literal), Unknown (Simple, Complex) \\ Figure 2.6: Initial Symbolic String Characteristics
}

In order to explain the initialization of an automata as a symbolic string variable, it is necessary to explain some characteristics of different string solution sets. When a string variable is initialized in a program, it will have three different characteristics: an alphabet, an initial bounding length, and a string type. Figure 2.6 lists each of these characteristics and briefly describes the characteristic.

A lphabet The alphabet of a symbolic string variable is most often specified as an input parameter to the PSE analysis. While individual symbols can be specified, it is often easier to specify a range of symbols in an existing symbol system such as the Unicode symbols. 
Initial Bounding Length The initial bounding length of a symbolic string variable is also usually specified as an input parameter to the PSE analysis. This bounding length $k$ is and upper bound on the initial length of strings in the solution set of symbolic string values. This bound is applied due to the exponential increase in solution set size for each linear increase in string length. To better demonstrate this increasing complexity, the solution set for a string with $\Sigma=\{\mathrm{A}, \mathrm{B}\}, k \leq 1$ is $\{\varepsilon, A, B\}$ where a similar solution set for a string with of $\Sigma=\{A, B\}, k \leq 2$ is $\{\varepsilon, A, B, A A, A B, B A, B B\}$, an increase in solution set size from 3 to 7 with only a corresponding length increase of 1 .

String Type The string type of a symbolic string value is a category assigned after observing the behavior of different string variables as symbolic strings in this analysis of symbolic string values. The three categories of string types are assigned based upon the membership of the solution set for the corresponding symbolic string variables and are the following string value types: null, concrete, and unknown. These categories can be further refined based on the corresponding automaton construction procedures and are the following five string types: empty, empty string, literal, simple unknown, and complex unknown. The empty string type is the only null value type and represents the the empty solution. This string type is only created when a string variable represents a null value, e.g. str $=$ null. The empty string string type is a concrete string value type and represents the solution set containing only the empty string. This string type is most often is generated as part of a predicate where a string is checked to determine if it is the empty string, e.g. implicitly created for str.isEmpty(), but can also often occur as string literals, e.g. str = "". A literal string type is a concrete string value type and represents the solution set containing only one non-empty string value. A literal type is most often used for string literals 


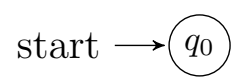

(a) Empty

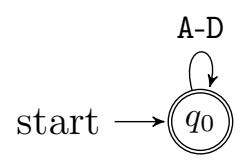

(d) Simple Unknown start $\longrightarrow q_{0}$

(b) Empty String

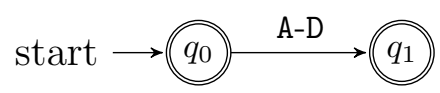

(e) Bound Automaton

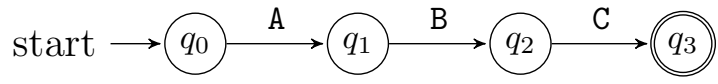

(c) Literal

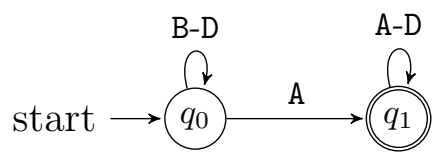

(f) Complex Unknown

Figure 2.7: FSA Creation Examples.

in a program, e.g. println("Hello World"). A simple unknown string type is an unknown string value type with a solution set containing more all possible string values for the specified alphabet and bounding length. The simple unknown string type is used when initializing a string variable, often from an input source external to the program under analysis, e.g. void func(String str). Finally, a complex unknown string type is an unknown string value type with a solution set containing more than one possible string value but not all possible string values for the specified alphabet and bounding length. The complex unknown string type usually occurs as the result of one or more string operations or as the result of applying a string predicate. Because of this additional complexity of only containing some of the possible string values in the solution set, the corresponding automaton representations of complex unknown string types have a much more complex structure than any of the other four types and are the main focus of this research of automata-based symbolic string constraints.

When initializing a FSA as a symbolic string value, the FSA is created in one of several different ways depending on the type of string represented. Figure 2.7 shows examples of six different automata representing the different construction methods for automata-based symbolic string models. Figure 2.7a is a single non-accepting 
state which is also the start state in the automata. This automaton is created for all empty string type variables. Figure $2.7 \mathrm{~b}$ shows an automaton consisting of only a single accepting start state. This automaton is created for all empty string string type variables. Figure $2.7 \mathrm{c}$ is an automaton which accepts only the string value ABC. This is an example automaton created for a literal string type variable. The construction of such an automaton begins with a non-accepting start state, $q_{0}$ in the example, and an additional state, $q_{1}$ in the example, is added to the automaton with a transition from $q_{0}$ to $q_{1}$ for the first symbol of the string value, $\mathrm{A}$ in the example. This process is repeated for each subsequent symbol in the string value until all the symbols of the string value are reflected in the automaton. To complete the literal type automaton, the last added state, $q_{3}$ in the example, is then made an accepting state. Figure $2.7 \mathrm{~d}$ is an automaton which accepts any string of any length in the with the alphabet $\Sigma=\{\mathrm{A}, \mathrm{B}, \mathrm{C}, \mathrm{D}\}$. This is an example automaton created for a simple unknown string type variable. The creation of such automata requires only a two part process. First, a single accepting start state is used. Then, a transition is added to the automaton from the start state to itself for every symbol in the alphabet $\Sigma$. Figure $2.7 \mathrm{e}$ is a bounded automaton which in this example is a modification of the previous simple unknown automaton in Figure 2.7d. This example automaton is bounded by an initial bounding length $k$ where $k \leq 1$. Bounded automata like this are useful in some analyses where in some operations and predicates an infinite automaton can not be used. Finally, Figure $2.7 \mathrm{f}$ is an automaton that accepts any non-empty string with the alphabet $\Sigma=\{\mathrm{A}, \mathrm{B}, \mathrm{C}, \mathrm{D}\}$ where the accepted string must contain the A symbol. This is just one example of an automaton representing a complex unknown string type variable. This string type does not have a defined construction since such types are only produced as a result from string operations and string predicates. To demonstrate this, the 
example automaton in Figure 2.7f was constructed by asserting the Contains(A) predicate for a simple unknown string type for the alphabet $\Sigma=\{\mathrm{A}, \mathrm{B}, \mathrm{C}, \mathrm{D}\}$ rather than through any specific construction procedure. While complex unknown string type variables are important to understanding how

Both following the creation of an automaton as symbolic string models and following any string operations or string predicates, an automaton is evaluated for determinism and converted into a deterministic automaton if it is not already. Then the automaton is minimized. This minimal DFA is optimized for space by utilizing the fewest number of states and transitions possible to accept the language of the automaton. Additionally, the minimal DFA is optimized for future algorithms which emulate string operations and predicates since the performance complexity of those algorithms is dependent upon the number of states and transitions in automata.

\section{String Operations}

\begin{tabular}{|c|c|}
\hline Category: & Examples \\
\hline Injective & ToString $(s t r)$, ReVERSE $(s t r)$ \\
\hline Additive & Concatenate $\left(s t r_{1}, s t r_{2}\right), \operatorname{InSERT}\left(s t r_{1}, i n t, s t r_{2}\right)$ \\
\hline Subtractive & DELETE $\left(s t r_{1}, i n t_{1}, i n t_{2}\right)$, SUBSTRING $\left(s t r_{1}, i n t_{1}, i n t_{2}\right)$ \\
\hline Substitutive & REPLACE $\left(s t r_{1}, \operatorname{char}_{1}, \operatorname{char}_{2}\right), \operatorname{REPLACE}\left(s t r_{1}, s t r_{2}, s t r_{3}\right)$ \\
\hline Mixed Constraint & LENGTH(str), INDEXOF(char) \\
\hline Infeasible & Format $(s t r, o b j[]), \operatorname{HASHCODE}(s t r)$ \\
\hline
\end{tabular}

Figure 2.8: String Operation Categories

In order to discuss the simulating of string operations for automata-based symbolic string models, six categories of string operations will first be explored. We assigned these six categories to the different string operations we encountered in this analysis of automata-based symbolic string models. These six categories are as follows: injective, 
additive, subtractive, substitutive, mixed constraint, and infeasible. These categories are listed with example operations in the table in Figure 2.8. For the purposes of this discussion, the string variable upon which the string operation is being performed is always specified as the primary string argument for an operation.

Injective We identified injective string operations which are characterized by the guaranteed one-to-one correspondence between the string values in the solution sets of the resulting string and the primary string argument of the operation. In practice, only the most simple of string operations have been identified as injective operations. An example of an injective string operation is the $\operatorname{ToSTRING}(s t r)$ operation where the string returns a string representation of its value which is just the string itself for a string data type. A more important example of an injective string operation is the assignment of a string value to a string variable. When this type of operation is simulated for automata-based symbolic string models, the algorithms simply create a copy of the primary string argument automaton and returns the copy as the result of the operation.

Additive We identified additive string operations where additional symbols appear in the string returned by the operation compared to the primary string argument. This category of operation does not discriminate based upon where in the primary string argument the new symbols have been added. For example, the operation ConcAtenate $\left(s t r_{1}, s t r_{2}\right)$ adds the new symbols of $s t r_{2}$ after the existing symbols of the primary string argument $s t r_{1}$. However, the $\operatorname{InSERT}\left(s t r_{1}, i n t, s t r_{2}\right)$ operation adds the new symbols of $s t r_{2}$ to the primary string argument $s t r_{1}$ as the index specified by int. Both of these example operations add new symbols to primary string argument, but not necessarily at the same location within the string. While these differences in additive operations requires different simulating algorithms for each operation instead 
of relying on a single algorithm as is the case with injective operations.

Subtractive We identified subtractive string operations where symbols have been removed in the string returned by the operation compared to the primary string argument. This category of operation includes all operations which remove symbols from the primary string argument regardless of the location at which the symbols are removed. For example, the $\operatorname{DELETE}\left(s r_{1}, i n t_{1}, i n t_{2}\right)$ operation removes all the symbols in the primary string argument $s t r_{1}$ from the index specified by the first integer argument $i n t_{1}$ to the index specified by the second integer argument $i n t_{2}$. While the SUBSTRING $\left(s t r_{1}, i n t_{1}, i n t_{2}\right)$ operation accepts the same arguments, it removes all symbols from the primary string argument $s t r_{1}$ both before the index specified by the first integer argument $i n t_{1}$ and after the second integer argument $i n t_{2}$. Just as with additive operations, simulating subtractive operations requires unique algorithms for each specific operation.

Substitutive We identified substitutive string operations where symbols have been substituted in the string returned by the operation compared to the primary string argument. There are two sub-types of substitutive string operations: simple and complex. The simple variant of the substitutive operation performs the substitution of symbols without altering the length of the string, i.e., in a simple substitutive operation, the lengths of the primary string argument and the string resulting from the operation are the equal. An example of this is the $\operatorname{REPLACE}\left(\operatorname{str}_{1}, \operatorname{char}_{1}, \operatorname{char}_{2}\right)$ operation where all instances of the first symbol argument char $_{1}$ in the primary string argument $s t r_{1}$ are replaced with the second symbol argument char $_{2}$. Because individual symbols are being replaced by other individual symbols in the primary string, the total length of the string does not change. The complex variant of the substitutive operation performs the substitution of symbols without being able 
to guarantee that the length of the string will be unaltered, i.e., in a complex substitutive operation, the lengths of the primary string argument and the string resulting form the operation may or may not be equal. An example of this is the $\operatorname{REPLACE}\left(s t r_{1}, s t r_{2}, s t r_{3}\right)$ operation all instances of the second string argument $s t r_{2}$ within the primary string argument $s r_{1}$ are substituted for the third string argument $s r_{3}$. Because the lengths of the second and third string arguments may differ, the replacement of the substrings within the primary string can result in string with a different length. Despite the differences between the simple and complex substitutive operations, the simulation algorithms for these operations are similar enough to group them within the same category as we have done.

Mixed Constraint We identified mixed constraint string operations where the result of the operation was not a string data type. An example of this operation type is the Length(str) operation which returns an integer value representing the length of the string. While the LENGTH(str) operation could simulated by a fairly simple algorithm, the return value would need to be a symbolic integer which could be represented by one of many different symbolic integer models. Any algorithm used to simulate a mixed constraint operation for automata-based symbolic strings would also need to be specialized to return a symbolic model for the return data type of the operation.

Infeasible We identified infeasible string operations where the complexity requirements of simulating the operation for automata-based symbolic string models is not feasible by any known algorithms. An example of such an operation is the Format $(s t r, o b j[])$ operation where the primary string argument automaton would need to be processed for a multitude of special character sequences for each possible string value in the solution set of the automaton. Similarly, the mixed constraint 


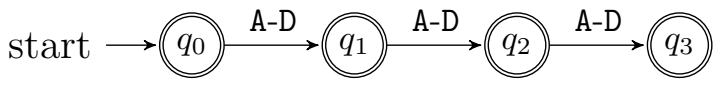

(a) Initial automaton

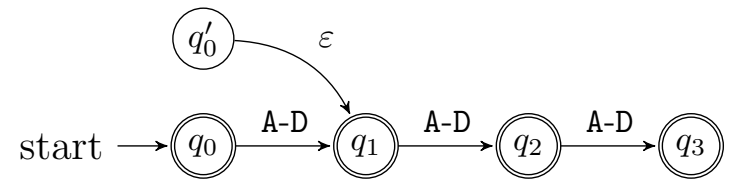

(c) Add $\varepsilon$-transitions to start

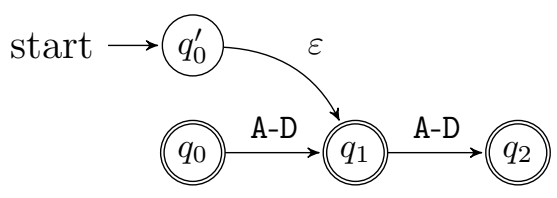

(e) Set $q_{0}^{\prime}$ as start state $q_{0}^{\prime}$

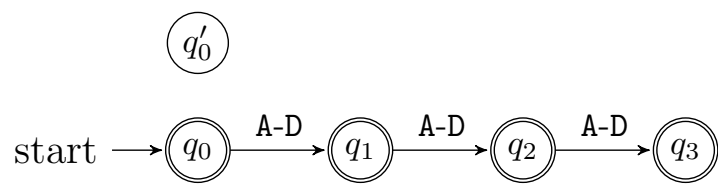

(b) Add new start state

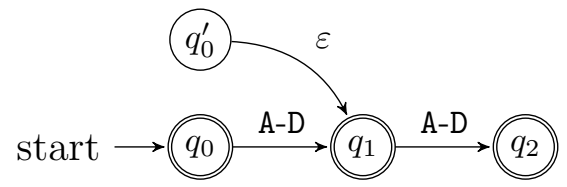

(d) Remove transitions after end

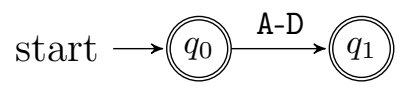

(f) Make deterministic and minimize

Figure 2.9: FSA $\operatorname{Substring}(\mathcal{A}, 1,2)$ operation example

operation $\operatorname{HAshCODE}(s t r)$ is also an infeasible operation due to the complexity required to compute the hash code for each possible string value in the primary string argument solution set.

Figure 2.9 demonstrates how a string operation can be simulated for an automatabased symbolic string model. In this example, the SUBSTRING operation with a start index of 1 and an end index of 2. This operation performed for a simple unknown string type variable, shown in Figure 2.9a, with an alphabet $\Sigma=\{\mathrm{A}, \mathrm{B}, \mathrm{C}, \mathrm{D}\}$ and initial bounding length $k \leq 3$. The first step to simulate the operation is the addition of a new state $q_{0}^{\prime}$ to the automaton as shown in Figure 2.9b. Next Figure 2.9c show that an epsilon-transition is created from the new state $q_{0}^{\prime}$ to the state at a length of 1 from the start state, the state $q_{1}$ in this case. This epsilon-transition represents the removal of the symbols not captured in the substring operation, i.e. the symbols occurring before the start index. Figure 2.9d illustraits the next step where the transitions 
leaving the states at the end length in the automaton have their outgoing transitions removed and are made accepting states if they are not already. This step emulates the removal of all symbols following the end index in original string. Figure 2.9e shows that the start state of the automaton is changed from the previous start state $q_{0}$ to the newly added start state $q_{0}^{\prime}$. Finally, Figure $2.9 \mathrm{f}$ is an automaton which is the result of making the previous automaton deterministic and then minimizing the resulting automton. It is in this manner that the SUBSTRING string operation is simulated so that an automaton accepting the language $\mathcal{L}(\mathcal{A})=\{\varepsilon, \mathrm{A}, \mathrm{B}, \mathrm{C}, \mathrm{D}\}$ is created and returned from the algorithm. Other string operation simulation algorithms operate similarly by altering the structure of automata, usually by adding states, adding and removing transitions, and adding or removing states from the set of accepting states.

1: procedure FASTSUBSTRING $(\mathcal{A})$

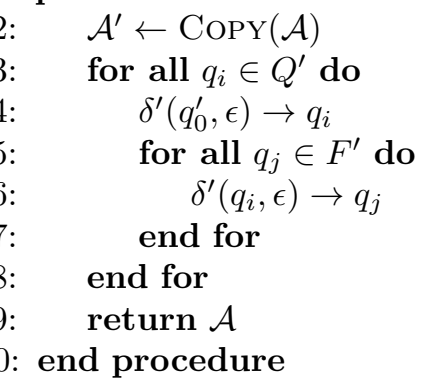

(a) Fast Substring Algorithm

: procedure FollowTransitions $(Q)$

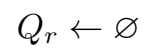

(b) FollowTransitions
1: procedure PRECISESUbSTRING $(\mathcal{A}$, start, end $)$ $\mathcal{A}^{\prime} \leftarrow \operatorname{Copy}(\mathcal{A})$

(c) Precise Substring Algorithm

Figure 2.10: Two SubSTRING operation algorithms 
An important consideration when selecting an appropriate simulation algorithm for a string operation is the balance of precision and performance of the algorithm. Most string operations can be simulated by many different algorithms some of which have less computational complexity at the cost of over-approximating the solution sets for the resulting automata-based symbolic string models. An example of this performance difference can be seen in the two algorithms for the SUBSTRING operation shown in Figure 2.10. The FAstSubstring (2.10a) algorithm does not utilize any of the indices which are arguments for a SUBSTRING operation, instead the algorithm returns an over-approximated automaton where the solution set contains substring values for all possible start and end indices to the SUBSTRING operation. The PRECISESUbSTRING (2.10c) algorithm makes use of the start and end indices to provide a more precise resulting automaton. This algorithm is the version of the SUBSTRING operation used in the previous example shown in Figure 2.9. This precision comes at the performance cost incurred by having to traverse along the transitions of the automaton up to the start and end lengths. It is clear that PRECISESubSTRING is an algorithm with much greater precision than the FASTSUBSTRING algorithm, but this precision does come at the cost of performance. The computational complexity of the FASTSUBSTRING algorithm is depends upon the number of states in the automaton and the number of those that are accepting due to the nested loops on lines 3 and 5. In comparison, the PreciseSubstring algorithm contains two loops on lines 4 and 9 with a call to the FollowTransitions (2.10b) sub-algorithm nested within the loop. This FollowTransitions sub-algorithm itself contains nested loops on lines 3 and 4 which result in a PreciseSubstring algorithm which contains two triple nested loops and two single loops on lines 14 and 17 . The improvement in computational complexity of the FASTSUBSTRING algorithm compared to the 
PreciseSubstring algorithm is clear, but at the cost of over-approximating the solution set.

There do exist some string operations which will always have the potential to over-approximate the solution set of a string variable. These are of subset of string operations under which regular languages are not closed. Because the languages accepted by automata are only regular languages, any operation under which regular languages are not closed cannot be precisely modeled by an automaton. Thus, when some string functions are modeled by an algorithm, the resulting automaton can be an over-approximation of the actual solution set. For example, some algorithms which model the REPLACE operation produce an over-approximated solution set [38,39]. The choice to use an over-approximating modeling algorithm is made because either it is much more efficient than a precise algorithm or no known precise modeling algorithm is known. Which approach to utilize when choosing a string operation simulation algorithm depends upon the analysis in which such algorithms are used.

\section{String Predicates}

\begin{tabular}{|c|c|}
\hline Category: & Examples \\
\hline Full Match & $s t r_{1}=s t r_{2}$, EQUALSIGNORECASE $\left(s t r_{1}, s t r_{2}\right)$ \\
\hline Partial Match & CONTAins $\left(s t r_{1}, s t r_{2}\right), \operatorname{StARTSWITH}\left(s t r_{1}, s t r_{2}\right)$ \\
\hline Mixed Constraint & $\operatorname{LENGTH}(s t r)>$ int, $\operatorname{IndexOF}($ char $)<i n t$ \\
\hline Infeasible & $\operatorname{MATCHES}\left(s t r_{1}, s t r_{\text {regex }}\right), s t r_{1}=\operatorname{FORMAT}\left(s t r_{2}, o b j[]\right)$ \\
\hline
\end{tabular}

Figure 2.11: String Predicate Categories

In order to examine the application of string predicates for automata-based symbolic string models, four categories of string predicates must first be reviewed. We assigned these four categories to the different string predicates we encountered in this analysis of automata-based symbolic string models. These four categories are as 
follows: full match, partial match, mixed type, and infeasible. These categories are listed with example predicates in the table in Figure 2.11.

Full Match We identified full match string predicates where two string values are compared to determine equality. Some of these predicates include some prepossessing of the string arguments such that the string returned from this prepossessing is used in the equality comparison. An example of a string predicate which includes some prepossessing of the string arguments is the EQUALSIGNORECASE $\left(s r_{1}, s t r_{2}\right)$ predicate where both string arguments $s t r_{1}$ and $s t r_{2}$ have their symbols converted into the same case before being compared for equality. This is an extra step compared to the much simpler predicate $s t r_{1}=s t r_{2}$ which requires no prepossessing step before comparing $s t r_{1}$ and $s t r_{2}$ for equality. Full match string predicates are very simple to simulate for automata-based symbolic string models because the equality predicate is just the automaton INTERSECT operation which has many known algorithms which can be implemented [32]. Similarly, to check for inequality the INTERSECT operation is used between the first automaton and the automaton returned from the COMPLEMENT operation performed on the automaton of the second string.

Partial Match We identified partial match string predicates where two string values are compared to determine if a portion of the first string value is equal to either a second string value or a portion of a second string value. One example of this kind of predicate is Contains $\left(s t r_{1}, s t r_{2}\right)$ where the first string $s t r_{1}$ is checked to see if it contains a sequence of symbols equal to the second string $s t r_{2}$. Similarly, the partial match predicate $\operatorname{StARTSWITH}\left(s t r_{1}, s t r_{2}\right)$ where the first string $s t r_{1}$ is checked to see if it begins with the sequence of symbols equal to the second string $s t r_{2}$. Partial match string predicates like full match predicates are fairly simple to simulate for automata-based symbolic string models. Just like full match predicates, 
partial match predicates use the automaton INTERSECT operation to check for partial equality, however this check for partial equality requires the concatenation of SIMPLE UNKNOWN string automata such that the new automata is added to the beginning and/or the end of the second automaton in the predicate depending on the specific predicate used. The check for partial inequality for partial match predicates is handled similarly to full match types where the previously described concatenated second automaton is processed by the COMPLEMENT automaton operation before intersected with the first automaton argument.

Mixed Constraint We identified mixed constraint string predicates where the operations contained in the predicate where mixed constraint string operations. An example of this kind of predicate is $\operatorname{LENGTH}(s t r)>$ int where the length of a string value is compared to an integer value. Just like mixed constraint string operations, any algorithm which can simulate a mixed constraint string predicate would need to be specialized to handle both automata-based symbolic strings and symbolic values for the other data types included in the predicate.

Infeasible We identified infeasable string predicates where the complexity requirements of simulating the predicate for automata-based symbolic string models is not feasible by any known algorithms. This infeasibility can occur due to either the logic of the predicate itself being to complex or due to the inclusion of infeasable string operations in the predicate. An example of an infeasible complex predicate is $\operatorname{Matches}\left(s t r_{1}, s t r_{\text {regex }}\right)$ which would require the processing of the automaton representing the regular expression string to be processed for all the possible regular expressions such a string could have, a very complex task. An example of an infeasible predicate due to included infeasible operations would be $s r_{1}=\operatorname{FoRMAT}\left(s t r_{2}, o b j[]\right)$ where equality is checked between one string $s t r_{1}$ and a formatted string returned 
from the infeasible string operation $\operatorname{FORMAT}\left(s t r_{2}, o b j[]\right)$.

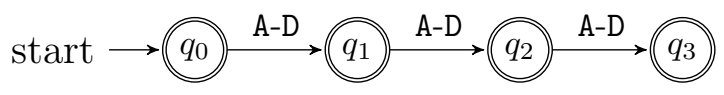

(a) Primary automaton

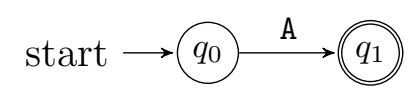

(b) A automaton

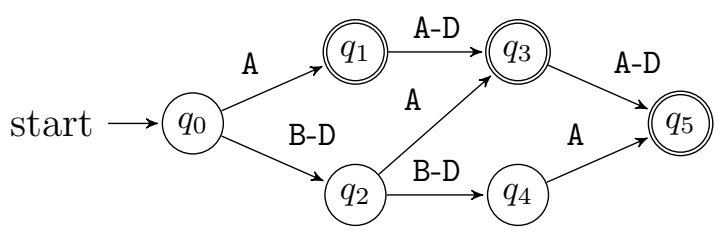

(d) Resulting automaton

Figure 2.12: FSA Contains $\left(\mathcal{A}_{1}, \mathrm{~A}\right)$ true predicate example

Figure 2.12 how a string predicate can be simulated for an automata-based symbolic string model. In this example, the true branch outcome is asserted for the $\operatorname{Contains}\left(\mathcal{A}_{1}, \mathrm{~A}\right)$ predicate. This predicate is performed for a simple unknown string type variable, shown in Figure 2.12a, with an alphabet $\Sigma=\{\mathrm{A}, \mathrm{B}, \mathrm{C}, \mathrm{D}\}$ and initial bounding length $k \leq 3$. Figure $2.12 \mathrm{~b}$ shows the literal string type automaton that is created for the A argument of the Contains predicate. In order for the simulate the predicate, the argument automaton must be modified to represent any string containing A so that it can be used in an INTERSECT automaton operation. Figure 2.12c illustrates how this is done by concatenating two simple unknown string type automata to the argument automaton, one preceding the argument automaton and one following it. Figure $2.12 \mathrm{~d}$ is the automaton which is returned by the intersection of the primary automaton (Figure 2.12a) and the prepared argument automaton (Figure 2.12c). This automaton accepts any string up to length 3 which also contains the symbol A which reflects only the string values for which the predicate $\operatorname{Contains}\left(\mathcal{A}_{1}, \mathrm{~A}\right)$ is true. 


\section{Model Counting}

When modeling string constraints with automata in a quantitative string analysis such as PSE, the MC must be calculated using the automaton quintuple. Fortunately, research has shown that model counting an automaton can be reduced to exactly counting the accepting paths of the automaton [1]. This means that a simple algorithm for model counting automata requires a straightforward traversal of the automata graph until no more transitions can be explored or up to a specified bounding length $k$ if the automaton is contains one or more infinite cycles. This MC procedure will be examined in detail in Chapter 3. The calculation of MC from an automata is an area of ongoing research aimed at reducing the performance cost of model counting automata $[1,27]$. While the automaton $\mathrm{MC}$ calculation procedure could be optimized for better performance, the additional performance cost that is required by even the most efficient algorithms when added to the other additional performance costs required for using a PSE analysis results in a much more costly analysis than an SE analysis. In addition to these performance issues, other problems have been observed during quantitative string analyses which can render such analyses invalid.

\subsubsection{Limitations}

The two significant limitations which have been observed in both our research and other existing research using automata in SE and PSE are the collapse problem as discussed in Section 2.3.2 and over-approximation as discussed in Section 2.4.2. The occurrence of either one of these problems in a quantitative analysis can be enough to alter the MC and the subsequent probability calculations which can result in an 
invalid PSE analysis.

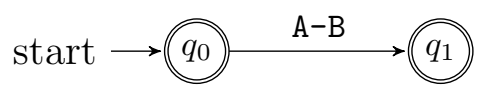

(a) FSA for $\Sigma=\{\mathrm{A}, \mathrm{B}\}$ and $k=1$.

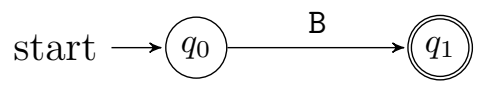

(c) $\mathrm{PC}_{4}$ Automaton

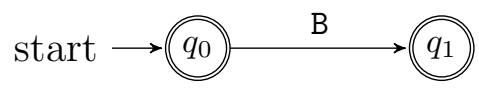

(b) FSA for $\operatorname{Replace}(X, \mathrm{~A}, \mathrm{~B})$

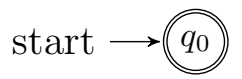

(d) $P C_{6}$ Automaton

Figure 2.13: An sample Java method that uses PSE with String variables.

To illustrate how the collapse problem occurs for automata-based symbolic string models, the example from Figure 2.4a will be revisited. The automata in Figure 2.13 correspond to the symbolic value $X$ shown in the PSE tree in Figure 2.4c. This automaton accepts $\varepsilon$ since the initial state $q_{0}$ is accepting and reaches the second accepting state $q_{1}$ by reading either an A or B symbol. The automaton therefore accepts three strings: $\varepsilon, A$, and B. When the initial string value undergoes a REPLACE operation on line 2, it produces the automaton shown in Figure 2.13a. This automaton accepts $\varepsilon$ since the initial state $q_{0}$ is accepting and reaches the second accepting state $q_{1}$ by reading a B symbol. This automaton therefore accepts only two strings: $\varepsilon$ and B. It is clear that the non-injective REPLACE operation produces an automaton which accepts fewer strings than the initial automaton, demonstrating the collapse problem for automaton-based symbolic string models. This is also seen in the automata for $P C_{4}$ and $P C_{6}$ in Figures $2.13 \mathrm{c}$ and $2.13 \mathrm{~d}$ respectively where both automaton only accept a single string for a total of two possible execution paths rather than the three which actually exist. 


\section{CHAPTER 3}

\section{AUTOMATA-BASED SYMBOLIC STRING MODELS}

This chapter details the theoretical work required to complete the analysis of automata-based symbolic string models. An automata-based symbolic string model, shortened as automata model and denoted with the symbol $\mathcal{M}$, encapsulates one or more FSAs as well as any other important data and data structures required to represent a solution set. Two instances of important data for each of the four automata models used in this analysis are the alphabet and the length of the automaton. The alphabet of an automata model is used to represent all the symbols possible in a quantitative analysis rather than just the symbols appearing in the solution set of the symbolic string. These additional symbols in the alphabet are needed to simulate string operations and predicates which require the construction of automata representing every possible symbol rather than just the subset of symbols which appear in the language of a specific automaton. The length of an automata model is used differently depending on the version of the automata model but ultimately is used to ensure the finite length of the automata when used in algorithms for string operations, string predicates, or model counting. The automaton structure itself remains the same for each type of automata model, using the quintuple defined in Section 2.4.1.

For our analysis of automata-based symbolic string models we utilized four distinct 
versions of automata models: unbounded, bounded, aggregate bounded, and weightedtransition aggregate. Section 3.1 details the unbounded automata model which serves as the basis for comparison to the other modified automata models. Section 3.2 describes the bounded automata model which is a fairly simple modification of the unbounded version. Section 3.3 describes the aggregate bounded automata model which is a further modification of the bounded version. Finally, Section 3.4 covers the weighted-transition aggregate automata model which incorporates a redesigned FSA using weighted-transitions as a further refinement of the aggregate automata model. Each of these automata models will be defined and three important uses of the automata model will be examined: model counting the automata model, string operations which result in an over-approximation of the solution set for the automata model, and string operations which result in a collapse problem for the automata model.

\subsection{Unbounded Automata Model}

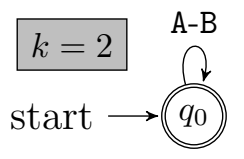

Figure 3.1: Unbounded Automata Model:

$$
\Sigma=\{\mathrm{A}, \mathrm{B}\}, k=2
$$

The unbounded automata model consists of a FSA, an alphabet, and a length. Figure 3.1 is an example of such an automata model with an alphabet $\Sigma=\{A, B\}$ and a length value $k=2$. The length value of the unbounded automata model is used as a maximum length counter when traversing automata when performing algorithms to ensure that automaton cycles do not cause endless loops. The maximum length value 
must be modified as part of additive, subtractive, and complex substitutive string operations to accurately reflect the way these operations alter the lengths of the resulting strings. The creation process for the FSA component of the unbounded automata model is completed as described in Section 2.4.2 for each of the empty, empty string, literal, simple unknown, and complex unknown string types. The unbounded automata model is equivalent to the automata models used in known string analysis research $[1,7,18,19,34,36-41]$.

\subsubsection{Model Counting}

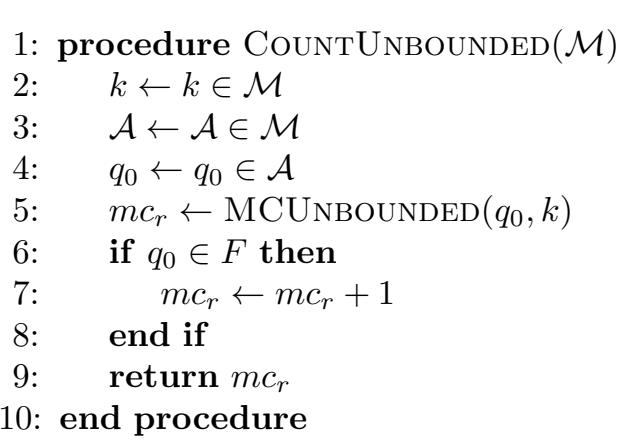

(a) Coordinating algorithm

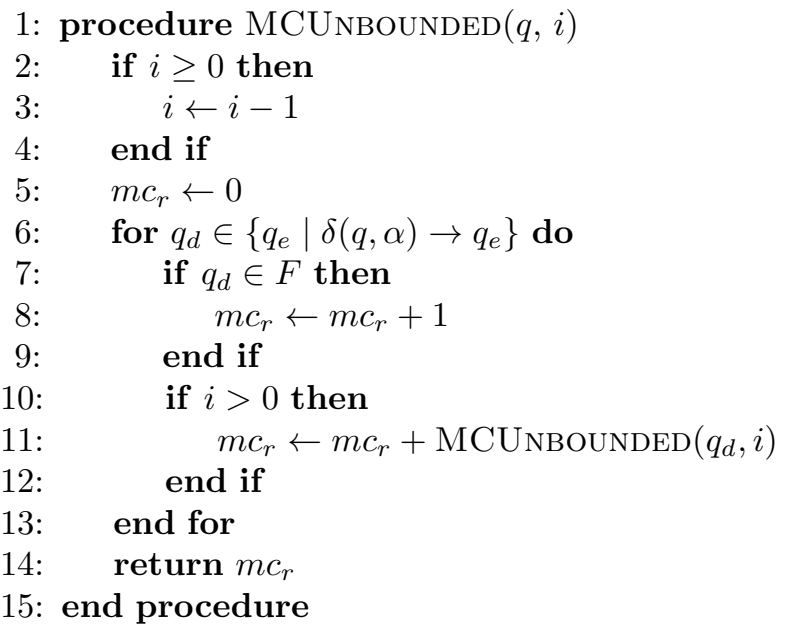

(b) Recursive model counting algorithm

Figure 3.2: Model counting algorithms for unbounded automata models

Figure 3.2 shows the model counting algorithms for the unbounded automata models where the coordinating algorithm is Figure 3.2a and the recursive algorithm is Figure 3.2b. To count an unbounded automata model $\mathcal{M}$, the model is passed as the argument to the coordinating CountUnBOunded algorithm. Next, the length $k$ and the start state $q_{0}$ of the FSA $\mathcal{A}$ are retrieved for the call of the MCUNBOUNDED recursive algorithm. The initial call to this algorithm is made using the start state 
$q_{0}$ as the state argument $q$ and using the length value $k$ as the counter $i$. Lines 1-3 of MCUnBOUNDED decrements the counter $i$ so that the recursive loops of this algorithm will not continue endlessly. Line 4 initializes the model count value to be returned by the algorithm $m c_{r}$. Line 5 of MCUNBOUNDED begins an iteration through all the outgoing transitions of the state $q$ provided as an input argument to the algorithm, the tuple $\left\langle\alpha_{t}, q_{d}\right\rangle$ which are the symbol and destination state of each outgoing transition are the values used in this for loop. Lines 6-8 of MCUNBOUNDED check to see if the transition destination state $q_{d}$ is an accepting state, i.e. $q_{d} \in F$. If $q_{d}$ is accepting, then Line 7 of MCUNBOUNDED increments the currently tracked model count $m c_{r}$ of transitions outgoing from the initial state $q$. Next, lines 9-11 of MCUNBOUNDED check the counter $i$ to determine if recursion should continue. If so, the result of a recursive call to the MCUNBOUNDED algorithm is added to the currently tracked number of model counts $m c_{r}$. The recursive call to the algorithm on line 10 of MCUNBOUNDED is made specifying the transition destination state $q_{d}$ and the updated length counter $i$. As a result of the loop covering Lines 5-12 of MCUnBOUNDED, the number of strings accepted by the automaton up to length $i$ for outgoing transitions from the state $q$ will be tracked by the $m c_{r}$ variable and is returned from the algorithm on Line 13 of MCUNBOUnDED. When the recursive calls to MCUnBounded finally complete, line 5 of CountUnBounded stores the returned model count as $m c_{r}$. Lines 6-8 of CounTUNBOUNDED then check if the start state $q_{0}$ is accepting and increments the model count $m c_{r}$ if so, this accounts for the possible empty string value.

While there is ongoing research into more efficient model counting algorithms for automata $[1,27]$ as discussed in Section 2.4.2, the straightforward and easy to understand Algorithm 3.2 is sufficient for our analysis of automata-based symbolic 
string models. This particular model counting algorithm will serve as a basis of comparison when discussing the corresponding algorithms for bounded, aggregate, and weighted automata models.

\subsubsection{Over-Approximation}

The over-approximation of a solution set due to the string operation simulating algorithms has been discussed previously in Sections 2.4.2 and 2.4.3. However, there is another possible source of over-approximation of a solution set for automata-based symbolic string models, the composition automata model itself. The string operation Concatenation is used to demonstrate this type of over-approximation for an unbounded automata model. The later Sections 3.2.2, 3.3.3, and 3.4.5 will examine how the model caused over-approximation does or does not occur for bounded, aggregate, and weighted automata models for the same CONCATENATION operation scenario.

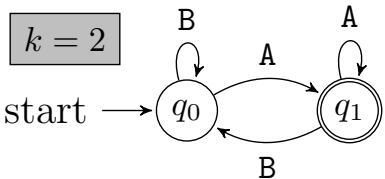

(a) $\mathcal{M}_{1}$ unbounded automata model

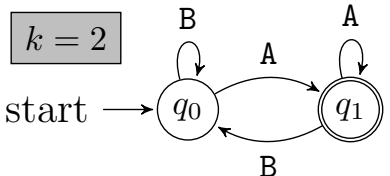

(b) $\mathcal{M}_{2}$ unbounded automata model

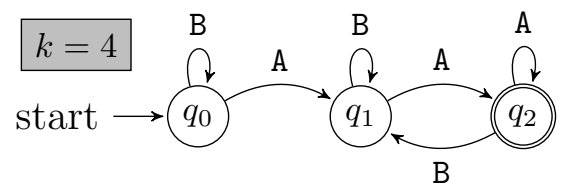

(c) $\mathcal{M}_{C}$ unbounded automata model resulting from CONCATENATION operation

Figure 3.3: Concatenation $\left(\mathcal{M}_{1}, \mathcal{M}_{2}\right)$ example for unbounded automata model

The example shown in Figure 3.3 demonstrates how over-approximation due to model structure can occur for unbounded automata models. This example presents 
the string operation CONCATENATION $\left(s t r_{1}, s t r_{2}\right)$ simulated for unbounded automata models $\mathcal{M}_{1}$ and $\mathcal{M}_{2}$ representing the string variables $s t r_{1}$ and $s t r_{2}$ respectively. Figure 3.3a shows the model diagram corresponding to $\mathcal{M}_{1}$ which accepts the language of strings up to a length 2 which contain only the symbols A and B and must end in the symbol $\mathrm{A}$, i.e., $\mathcal{M}_{1}$ has an alphabet $\Sigma_{\mathcal{M}_{1}}=\{\mathrm{A}, \mathrm{B}\}$ and maximum length $k_{\mathcal{M}_{1}}=2$ and represents the solution set $\mathcal{S}_{\mathcal{M}_{1}}=\{\mathrm{A}, \mathrm{AA}, \mathrm{BA}\}$. This unbounded automata model structure can appear often in a software program by asserting the $\operatorname{EndsWith}(\mathcal{M}, \mathrm{A})$ predicate. Figure $3.3 \mathrm{~b}$ shows the model diagram corresponding to $\mathcal{M}_{2}$ which has an identical structure to the $\mathcal{M}_{1}$ unbounded automata model, i.e., $\Sigma_{\mathcal{M}_{2}}=\{\mathrm{A}, \mathrm{B}\}, k_{\mathcal{M}_{2}}=2$, and $\mathcal{S}_{\mathcal{M}_{2}}=\{\mathrm{A}, \mathrm{AA}, \mathrm{BA}\}$. Figure 3.3c shows the diagram of the unbounded automata model $\mathcal{M}_{C}$ which is the result of the Concatenation operation. This resulting model has the same alphabet as both the $\mathcal{M}_{1}$ and $\mathcal{M}_{2}$ models since the alphabet of the automata model resulting from the CONCATENATION operation will be the UNION of the two argument automata models. The maximum length value of the $\mathcal{M}_{C}$ model has been set as $k_{\mathcal{M}_{C}}=4$ to reflect the addition of two length 2 automata models in the additive string operation. The cause of the over-approximation for the $\mathcal{M}_{C}$ unbounded automata model is this maximum length value and the fact that the length is tracked separately from the FSA. This can be seen by comparing the expected solution set $\mathcal{S}_{e}$ and actual solution sets for the $\mathcal{M}_{C}$ model $\mathcal{S}_{\mathcal{M}_{2}}$. It is expected that the Concatenation of $\mathcal{M}_{1}$ with solution set $\mathcal{S}_{\mathcal{M}_{1}}=\{\mathrm{A}, \mathrm{AA}, \mathrm{BA}\}$ and $\mathcal{M}_{2}$ with solution set $\mathcal{S}_{\mathcal{M}_{2}}=\{\mathrm{A}, \mathrm{AA}, \mathrm{BA}\}$ will produce the solution set $\mathcal{S}_{e}=\{\mathrm{AA}, \mathrm{AAA}, \mathrm{ABA}, \mathrm{BAA}, \mathrm{AAAA}, \mathrm{AABA}, \mathrm{BAAA}, \mathrm{BABA}\}$ which has a model count $M C\left(\mathcal{S}_{e}\right)=8$. However, the actual solution set produced for the $\mathcal{M}_{C}$ unbounded automata model is $\mathcal{S}_{a}=\{\mathrm{AA}, \mathrm{AAA}, \mathrm{ABA}, \mathrm{BAA}, \mathrm{AAAA}, \mathrm{AABA}, \mathrm{BAAA}, \mathrm{BABA}, \mathrm{ABAA}, \mathrm{ABBA}, \mathrm{BBAA}\}$ which has a model count $M C\left(\mathcal{S}_{\mathcal{M}_{C}}\right)=11$. This over-approximation occurs to the 
inclusion of the three string values ABAA, ABBA, and BBAA which cannot be produced from the Concatenation of $\mathcal{M}_{1}$ and $\mathcal{M}_{2}$ since $\mathrm{AB} \notin \mathcal{S}_{\mathcal{M}_{1}}$ and $\mathrm{BB} \notin \mathcal{S}_{\mathcal{M}_{1}}$. These

three string values only exist in the actual solution set $\mathcal{S}_{\mathcal{M}_{C}}$ due to the concatenation of the $\mathcal{M}_{1}$ and $\mathcal{M}_{2}$ automata as infinite automata with a independently tracked length value. Because this type of over-approximation for unbounded automata models is due to the alteration of maximum length value independent of the FSA, it is possible to similarly over-approximate the resulting models for any of the additive, subtractive, or complex substitutive string operations.

\subsubsection{Collapse}

In addition to the problems of over-approximation the unbounded automata model suffers from, the model is also susceptible to collapse problems as described in Sections 2.2.3 and 2.3.2. These collapse problems can manifest as the result of either subtractive or substitutive (both simple and complex) string operations. The DELETE operation is demonstrated to illustrate how collapse problems can occur due to the use of subtractive operations for unbounded automata models. Similarly, the REPLACE operation is examined to explain how collapse problems can occur when simulating substitutive operations for unbounded automata models. The specific REPLACE operation chosen for this example is the simple substitutive version of the operation $\operatorname{REPLACE}\left(\mathcal{M}, \alpha_{1}, \alpha_{2}\right)$ where $\alpha_{1}$ is a single symbol to be replaced in the primary string and $\alpha_{2}$ is a single symbol which replaces the $\alpha_{1}$ symbols in the resulting string. The later Sections 3.2.3, 3.3.4, and 3.4.6 will examine how the collapse problem does or does not occur for bounded, aggregate, and weighted automata models in the same Delete and Replace scenarios.

The example shown in Figure 3.4 demonstrates how the collapse problem can occur 


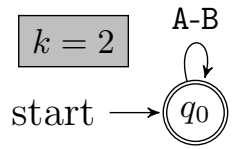

(a) $\mathcal{M}_{1}$ unbounded automata models

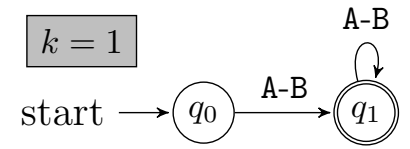

(b) $\mathcal{M}_{D}$ unbounded automata model resulting from DELETE operation

Figure 3.4: $\operatorname{DeLETE}\left(\mathcal{M}_{1}, 1,2\right)$ example for unbounded automata model

for unbounded automata models due to subtractive string operations. This example presents the string operation $\operatorname{DeLETE}\left(s t r_{1}, i n t_{s}, i n t_{e}\right)$ simulated for the unbounded automata model $\mathcal{M}_{1}$ and the start and end indices 1 and 2. Figure 3.4a shows the diagram corresponding to $\mathcal{M}_{1}$ such that $\mathcal{M}_{1}$ has an alphabet $\Sigma_{\mathcal{M}_{1}}=\{\mathrm{A}, \mathrm{B}\}$, a maximum length $k_{\mathcal{M}_{1}}=2$, and represents the solution set $\mathcal{S}_{\mathcal{M}_{1}}=\{\varepsilon, \mathrm{A}, \mathrm{B}, \mathrm{AA}, \mathrm{AB}, \mathrm{BA}, \mathrm{BB}\}$. Figure $3.4 \mathrm{~b}$ shows the diagram of the unbounded automata model $\mathcal{M}_{D}$ which is returned from the Delete $\left(\mathcal{M}_{1}, 1,2\right)$ operation. This automata model $\mathcal{M}_{D}$ has the same alphabet as $\mathcal{M}_{1}$ such that $\Sigma_{\mathcal{M}_{D}}=\{\mathrm{A}, \mathrm{B}\}$. The maximum length value $k$ for $\mathcal{M}_{D}$ has been reduced to $k=1$ to reflect the deletion of symbols. The collapse problem is identified when comparing the model count for the original automata model $M C\left(\mathcal{M}_{1}\right)=7$ to the model count of the resulting automata model $M C\left(\mathcal{M}_{D}\right)=2$ instead of the expected model count value of 6 (the empty string $\varepsilon$ does not contain a symbol at index 1 and therefore would return an error from the $\operatorname{DELETE}\left(\operatorname{str}_{1}, 1,2\right)$ operation). Because of the DELETE operation, each of the A, AA, and AB string values in $\mathcal{S}_{\mathcal{M}_{1}}$ correlates to the single A string value in $\mathcal{S}_{\mathcal{M}_{D}}$. The same relationship exists for the B, BA, and BB string values in $\mathcal{S}_{\mathcal{M}_{1}}$ and the single B string value in $\mathcal{S}_{\mathcal{M}_{D}}$. Thus, the original solution set values $\mathcal{S}_{\mathcal{M}_{1}}=\{\varepsilon, \mathrm{A}, \mathrm{B}, \mathrm{AA}, \mathrm{AB}, \mathrm{BA}, \mathrm{BB}\}$ collapse into the solution set values $\mathcal{S}_{\mathcal{M}_{D}}=\{\mathrm{A}, \mathrm{B}\}$ as a result of the $\operatorname{DeLete}\left(\mathcal{M}_{1}, 1,2\right)$ operation. This particular type of collapse behavior for unbounded automata models can occur for any subtractive string operation. 


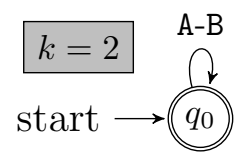

(a) $\mathcal{M}_{1}$ unbounded automata model

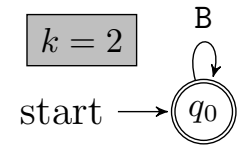

(b) $\mathcal{M}_{R}$ unbounded automata model resulting from REPLACE operation

Figure 3.5: $\operatorname{ReplacE}\left(\mathcal{M}_{1}, \mathrm{~A}, \mathrm{~B}\right)$ example for unbounded automata model

The example shown in Figure 3.5 demonstrates how the collapse problem can occur for unbounded automata models due to substitutive string operations. This example presents the string operation $\operatorname{REPLACE}\left(s t r_{1}, \operatorname{char}_{1}, \operatorname{char}_{2}\right)$ simulated for the unbounded automata model $\mathcal{M}_{1}$ representing the string argument $s r_{1}$ and the symbols A and B as the symbol arguments char $_{1}$ and char $_{2}$. Figure 3.5a shows the diagram corresponding to $\mathcal{M}_{1}$ such that $\mathcal{M}_{1}$ has an alphabet $\Sigma_{\mathcal{M}_{1}}=\{\mathrm{A}, \mathrm{B}\}$, a maximum length $k_{\mathcal{M}_{1}}=2$, and represents the solution set $\mathcal{S}_{\mathcal{M}_{1}}=\{\varepsilon, \mathrm{A}, \mathrm{B}, \mathrm{AA}, \mathrm{AB}, \mathrm{BA}, \mathrm{BB}\}$. Figure 3.4b shows the diagram of the unbounded automata model $\mathcal{M}_{R}$ which is returned from the $\operatorname{RePlaCE}\left(\mathcal{M}_{1}, \mathrm{~A}, \mathrm{~B}\right)$ operation. This automata model $\mathcal{M}_{R}$ has the same maximum length as $\mathcal{M}_{1}, k_{\mathcal{M}_{R}}=2$, since the symbols to be replaced (A) and the replacing symbols (B) are both single symbols. However, the alphabet of the resulting model only contains a single symbol $\Sigma_{\mathcal{M}_{R}}=\{\mathrm{B}\}$ since all A symbols were replaced in the operation. The collapse problem can be seen when comparing the model count of the original automata model $M C\left(\mathcal{M}_{1}\right)=7$ to the model count of the resulting automata model $M C\left(\mathcal{M}_{R}\right)=3$. Due to the Replace operation, both the A and B string values in $\mathcal{S}_{\mathcal{M}_{1}}$ correlate to the B string value in $\mathcal{S}_{\mathcal{M}_{R}}$. Similarly, each of the string values $\mathrm{AA}, \mathrm{AB}, \mathrm{BA}$, and $\mathrm{BB}$ in $\mathcal{S}_{\mathcal{M}_{1}}$ correlate to the BB string value in $\mathcal{S}_{\mathcal{M}_{R}}$. The empty string value $\varepsilon$ in $\mathcal{S}_{\mathcal{M}_{1}}$ correlates to the empty string value in the result solution set $\mathcal{S}_{\mathcal{M}_{R}}$. Thus, the original solution set values $\mathcal{S}_{\mathcal{M}_{1}}=\{\varepsilon, \mathrm{A}, \mathrm{B}, \mathrm{AA}, \mathrm{AB}, \mathrm{BA}, \mathrm{BB}\}$ collapse into the solution set values $\mathcal{S}_{\mathcal{M}_{R}}=\{\varepsilon, \mathrm{B}, \mathrm{BB}\}$ as a result of the $\operatorname{RePLACE}\left(\mathcal{M}_{1}, \mathrm{~A}, \mathrm{~B}\right)$ operation. 
This type of collapse behavior for unbounded automata models can occur for any substitutive string operations.

\subsection{Bounded Automata Model}

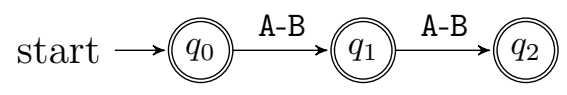

Figure 3.6: Bounded Automata Model:

$$
\Sigma=\{\mathrm{A}, \mathrm{B}\}, k=2
$$

The bounded automata model was created to solve the issue of model overapproximation as described in Section 3.1.2 for the unbounded automata model. Figure 3.6 is an example of a bounded automata model with an alphabet $\Sigma=\{\mathrm{A}, \mathrm{B}\}$ and a length value $k=2$. The bounded automata model consists of a FSA, an alphabet, and a length. The length value for this model is used to bound the length of the FSA to ensure that no cycles can exist within FSA, this allows the bounded model to avoid the problem of tracking the maximum length value external to the FSA which caused the over-approximation for the unbounded model.

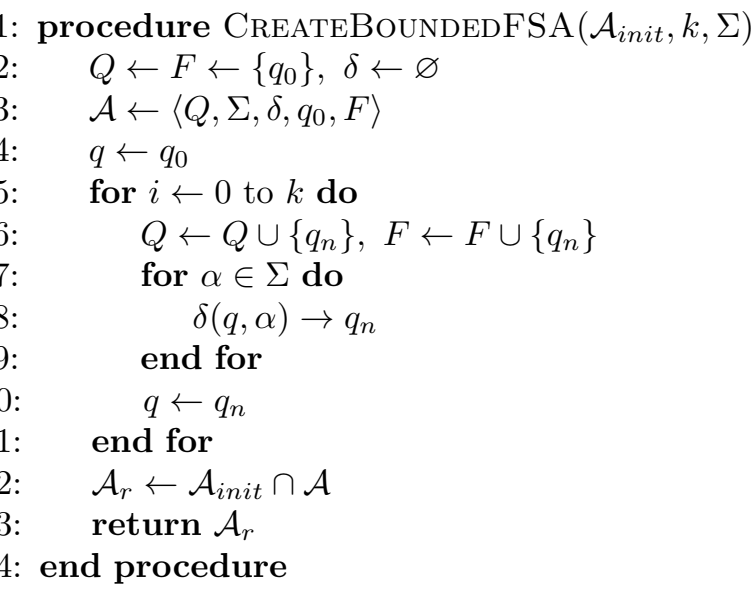

Figure 3.7: Creation algorithm for bounded automata model 
The FSA creation process for a bounded automata model begins just as the unbounded process, creating an initial FSA $\mathcal{A}_{\text {init }}$ as one of the five initial FSAs described in Section 2.4.2: empty, empty string, literal, simple unknown, and complex unknown. While only the unknown string types have a corresponding FSA which can contain cycles and would therefore need to be bounded by the length value $k$, the bounding process can still be performed for the other string type FSAs without issue. The bounding process is shown in Figure 3.7 where the initial FSA $\mathcal{A}_{\text {init }}$, the bounding length $k$, and the alphabet $\Sigma$ are required parameters. Lines 2 and 3 initialize the bounding FSA $\mathcal{A}$ with only the accepting start state $q_{0}$ which is then stored as the current state $q$ on line 4 . The loop on lines 5 -11 is used to create the bounding FSA which accepts all strings up to length $k$ in the alphabet $\Sigma$. Line 6 creates a new state $q_{n}$ and adds this state to the FSA as an accepting state. The loop on lines 7-9 creates a transition from the current state $q$ to the new state $q_{n}$ for every symbol $\alpha \in \Sigma$. On line 10 the current state $q_{b}$ is updated as the new state $q_{n}$ for the next iteration of the loop. After the loop is completed, the FSA $\mathcal{A}$ is now the desired bounding FSA accepting all strings up to the length $k$. Line 12 intersects the initial FSA $\mathcal{A}_{\text {init }}$ and the bounding FSA $\mathcal{A}$ to produce the desired bounded initial FSA $\mathcal{A}_{r}$. This FSA $\mathcal{A}_{r}$ has no cycles and accepts the same language as the initial FSA $\mathcal{A}_{\text {init }}$ such that $\mathcal{L}\left(\mathcal{A}_{i}\right)=\mathcal{L}\left(\mathcal{A}_{r}\right)$ for all strings with lengths less than or equal to the bounding length $k$. This bounded FSA $\mathcal{A}_{r}$ is added to the bounding length $k$ and the alphabet $\Sigma$ to complete the construction of the new bounded automata model.

\subsubsection{Model Counting}

Figure 3.8 shows the model counting algorithms for the bounded automata models where the coordinating algorithm is shown in Figure 3.8a and the recursive algorithm 


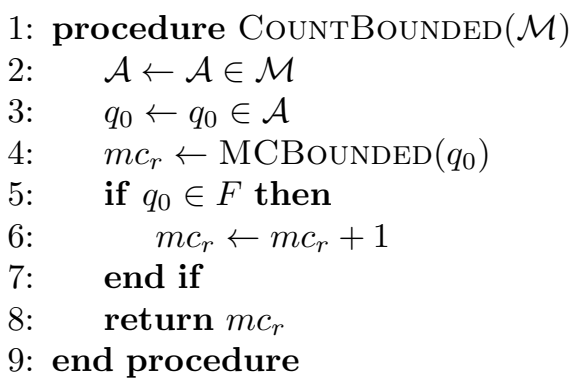

(a) Coordinating algorithm
1: procedure $\operatorname{MCBOunded}(q)$

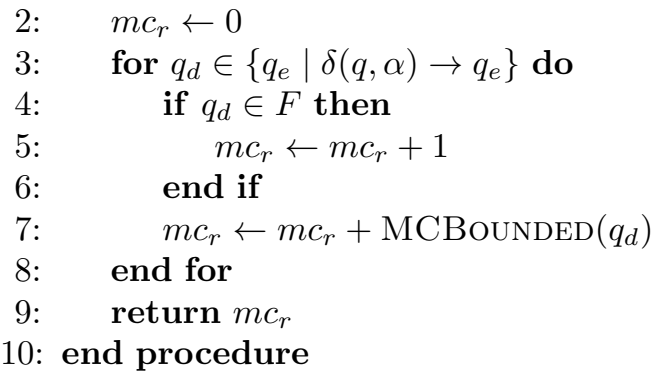

(b) Recursive model counting algorithm

Figure 3.8: Model counting algorithms for bounded automata models

is shown in Figure 3.8b. This version of the model counting algorithm works nearly the same as the unbounded version except that the bounded version does not include a counter parameter to the recursive algorithm since a bounded FSA cannot contain cycles.

\subsubsection{Over-Approximation}

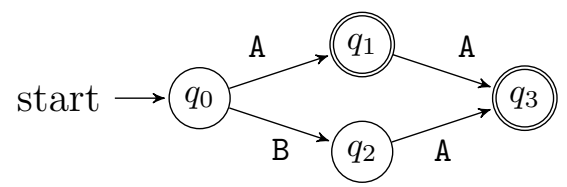

(a) $\mathcal{M}_{1}$ bounded automata model

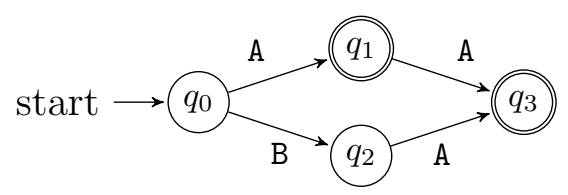

(b) $\mathcal{M}_{2}$ bounded automata model

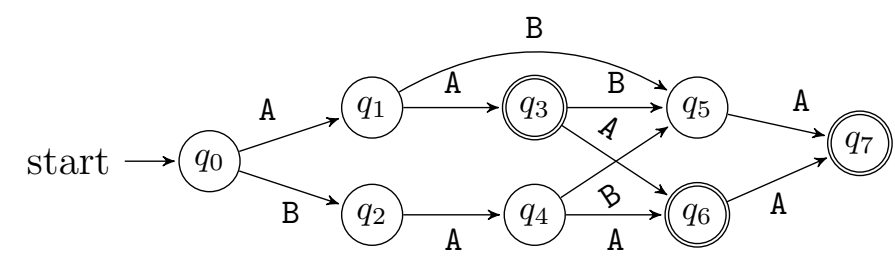

(c) $\mathcal{M}_{C}$ bounded automata model resulting from

Concatenation operation

Figure 3.9: Concatenation $\left(\mathcal{M}_{1}, \mathcal{M}_{2}\right)$ example for bounded automata model

The example shown in Figure 3.9 demonstrates how over-approximation due to 
model structure is prevented for bounded automata models. This example presents the string operation CONCATENATION $\left(s t r_{1}, s t r_{2}\right)$ simulated for bounded automata models $\mathcal{M}_{1}$ and $\mathcal{M}_{2}$ representing the string variables $s t r_{1}$ and $s t r_{2}$ respectively. Figures $3.9 \mathrm{a}$ and $3.9 \mathrm{~b}$ show the diagrams corresponding to $\mathcal{M}_{1}$ and $\mathcal{M}_{2}$ such that $\mathcal{M}_{1}$ and $\mathcal{M}_{2}$ both have an alphabet $\Sigma_{\mathcal{M}_{1}}=\Sigma_{\mathcal{M}_{2}}=\{\mathrm{A}, \mathrm{B}\}$ and bounding length $k_{\mathcal{M}_{1}}=k_{\mathcal{M}_{1}}=2$ and both represent the solution set $\mathcal{S}_{\mathcal{M}_{1}}=\mathcal{S}_{\mathcal{M}_{2}}=\{\mathrm{A}, \mathrm{AA}, \mathrm{BA}\}$. The string operation and automata model parameters are identical to those discussed in Section 3.1.2 for the unbounded automata model where over-approximation occurred. Figure 3.9c shows the diagram of the bounded automata model $\mathcal{M}_{C}$ which is the result of the CONCATENATION operation and has the same alphabets as $\mathcal{M}_{1}$ and $\mathcal{M}_{2}$, that is $\Sigma_{\mathcal{M}_{C}}=\{\mathrm{A}, \mathrm{B}\}$. This resulting model $\mathcal{M}_{C}$ has a solution set $\mathcal{S}_{\mathcal{M}_{\rfloor}}=\{\mathrm{AA}, \mathrm{AAA}, \mathrm{ABA}, \mathrm{BAA}, \mathrm{AAAA}, \mathrm{AABA}, \mathrm{BAAA}, \mathrm{BABA}\}$ which matches the expected solution set $\mathcal{S}_{e}=\{\mathrm{AA}, \mathrm{AAA}, \mathrm{ABA}, \mathrm{BAA}, \mathrm{AAAA}, \mathrm{AABA}, \mathrm{BAAA}, \mathrm{BABA}\}$ resulting from the $\operatorname{Concatenation}\left(s t r_{1}, s t r_{2}\right)$ operation the alphabet $\Sigma_{\mathcal{M}_{1}}=\Sigma_{\mathcal{M}_{2}}=\{\mathrm{A}, \mathrm{B}\}$ and bounding length $k_{\mathcal{M}_{1}}=k_{\mathcal{M}_{1}}=2$. Since the expected and actual solution sets are equivalent, the expected and actual model counts are equal, i.e. $M C\left(\mathcal{S}_{e}\right)=$ $M C\left(\mathcal{M}_{\rfloor}\right)=8$. Because the length is incorporated into the structure of the FSA by bounding the FSA in a bounded automata model, the over-approximation due to the model which can occur for unbounded automata models cannot occur for bounded automata models.

\subsubsection{Collapse}

While the bounded automata model prevents the over-approximation experienced by unbounded models, the collapse problems due to subtractive and substitutive operations still can occur for bounded automata models. 


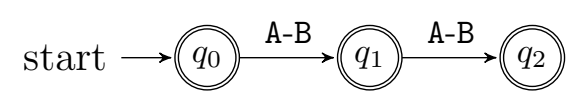

(a) $\mathcal{M}_{1}$ bounded automata model

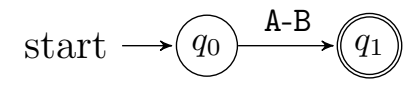

(b) $\mathcal{M}_{D}$ bounded automata model resulting from DeLETE operation

Figure 3.10: $\operatorname{DeLete}\left(\mathcal{M}_{1}, 1,2\right)$ example for bounded automata model

The example shown in Figure 3.10 demonstrates how the collapse problem can occur for bounded automata models due to subtractive string operations. This example presents the string operation $\operatorname{DELETE}\left(s t r_{1}, i n t_{s}, i n t_{e}\right)$ simulated for the bounded automata model $\mathcal{M}_{1}$ and the start and end indices 1 and 2. Figure 3.10a shows the diagram corresponding to $\mathcal{M}_{1}$ such that $\mathcal{M}_{1}$ has an alphabet $\Sigma_{\mathcal{M}_{1}}=\{\mathrm{A}, \mathrm{B}\}$ and maximum length $k=2$ and represents the solution set $\mathcal{S}_{\mathcal{M}_{1}}=\{\varepsilon, \mathrm{A}, \mathrm{B}, \mathrm{AA}, \mathrm{AB}, \mathrm{BA}, \mathrm{BB}\}$. Figure $3.10 \mathrm{~b}$ shows the diagram of the bounded automata model $\mathcal{M}_{D}$ which is returned from the $\operatorname{DeLetE}\left(\mathcal{M}_{1}, 1,2\right)$ operation and has the same alphabet as $\mathcal{M}_{1}$, $\Sigma_{\mathcal{M}_{D}}=\{\mathrm{A}, \mathrm{B}\}$. As with the unbounded DeLETE operation in Section 3.1.3, the solution set of the bounded automata model produced by simulating the DELETE operation $\mathcal{S}_{\mathcal{M}_{D}}=\{\mathrm{A}, \mathrm{B}\}$ and the corresponding model count $M C\left(\mathcal{M}_{D}\right)=2$ does not match the expected model count of 6 (the empty string should not be represented in the model after the operation). This example demonstrates that bounded automata models do not prevent collapses due to subtractive string operations.

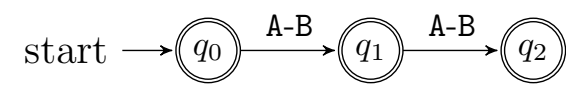

(a) $\mathcal{M}_{1}$ bounded automata model

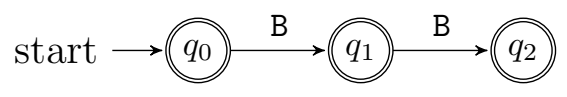

(b) $\mathcal{M}_{R}$ bounded automata model resulting from REPLACE operation

Figure 3.11: $\operatorname{RePlacE}\left(\mathcal{M}_{1}, \mathrm{~A}, \mathrm{~B}\right)$ example for bounded automata model

The example shown in Figure 3.11 demonstrates how the collapse problem can occur for bounded automata models due to substitutive string operations. This 
example presents the string operation $\operatorname{REPLACE}\left(s t r_{1}\right.$, char $_{1}$, char $\left._{2}\right)$ simulated for the bounded automata model $\mathcal{M}_{1}$ representing the string argument str $_{1}$ and the symbols A and B as the symbol arguments char $_{1}$ and char $_{2}$. Figure 3.11a shows the diagram corresponding to $\mathcal{M}_{1}$ such that $\mathcal{M}_{1}$ has an alphabet $\Sigma_{\mathcal{M}_{1}}=\{\mathrm{A}, \mathrm{B}\}$ and a bounding length $k_{\mathcal{M}_{1}}=2$ and represents the solution set $\mathcal{S}_{\mathcal{M}_{1}}=\{\varepsilon, \mathrm{A}, \mathrm{B}, \mathrm{AA}, \mathrm{AB}, \mathrm{BA}, \mathrm{BB}\}$. Figure $3.11 \mathrm{~b}$ shows the diagram of the bounded automata model $\mathcal{M}_{R}$ which is returned from the DeLete $\left(\mathcal{M}_{1}, 1,2\right)$ operation. As with the unbounded Replace operation in Section 3.1.3, the solution set for the bounded automata model produced by simulating the REPLACE operation $\mathcal{S}_{\mathcal{M}_{\nabla}}=\{\varepsilon, \mathrm{B}, \mathrm{B}\}$ and the corresponding model count $M C\left(\mathcal{M}_{R}\right)=3$ does not match the expected model count of 7 . As with the Delete example, this Replace example demonstrates that the bounded automata model does not prevent collapses due to substitutive string operations.

\subsection{Aggregate Bounded Automata Model}

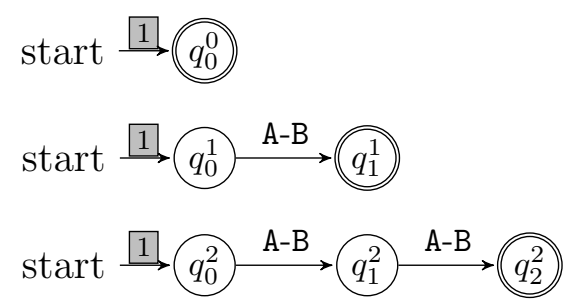

Figure 3.12: Aggregate Bounded Automata Model:

$$
\Sigma=\{\mathrm{A}, \mathrm{B}\}, k=2
$$

The aggregate bounded automata model, shortened henceforth as aggregate automata model, was created to prevent collapse problems which occur due to subtractive string operations as described in Sections 3.1.3 and 3.2.3. Figure 3.12 is an example of an aggregate automata model with an alphabet $\Sigma=\{\mathrm{A}, \mathrm{B}\}$ and a 
length value $k=2$. The aggregate model includes a sequence of FSAs, a sequence of positive integers called factors, an alphabet, and a length. The factors $\left(f_{n}\right)_{n=0}^{k}$ are updated in string operation simulation algorithms and are used as a multiplying factors when computing the model count of an aggregate model. As with the bounded model, the aggregate automata model uses the length value to bound the length of FSAs. However, unlike the bounded automata model, the aggregate automata model can contain more than one FSA. These multiple FSAs are collected into a sequence such that each FSA in the sequence correlates to a FSA which accepts a language of strings with precisely the length of the corresponding index in the sequence, i.e. $\left(\mathcal{A}_{n}\right)_{n=0}^{k}$ where $\mathcal{L}\left(\mathcal{A}_{n}\right)$ only contains strings of length $n$. In the example aggregate automata model, the FSA with start state $q_{0}^{0}$ is at index 0 in the FSA sequence and only accepts the empty string $\varepsilon$ which is a string with length 0 . Similarly, the FSA with start state $q_{0}^{1}$ is at index 1 in the FSA sequence and only accepts strings of length 1. The process of FSA creation is similar to that used for unbounded automata models, starting with the creation of an initial FSA $\mathcal{A}_{\text {init }}$ as described in Section 2.4.2.

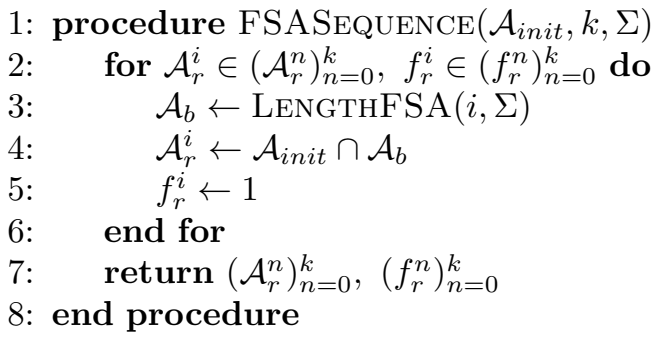

(a) Creation algorithm for FSA sequence

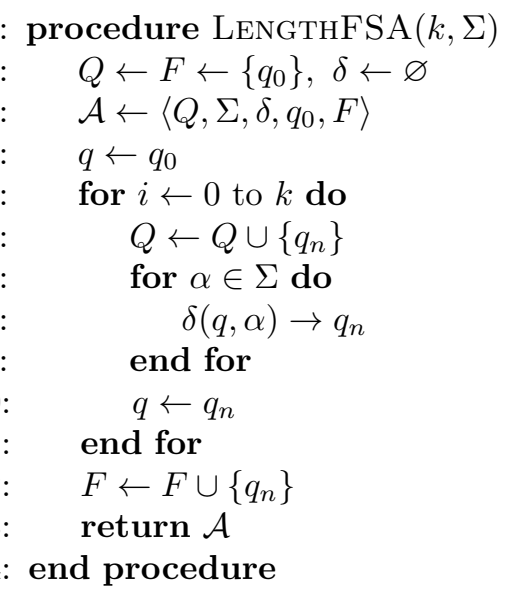

(b) Creation algorithm for length bounded FSA

Figure 3.13: Algorithms to create FSA sequence for aggregate automata model 
Figure 3.13 contains the algorithms used to create the FSA and factor sequences for an new aggregate automata model. The sequence creation process begins using the FSASEQUENCE algorithm shown in Figure 3.13a which requires an initial FSA $\mathcal{A}_{\text {init }}$, an initial bounding length $k$, and an alphabet $\Sigma$ as parameters. Lines 2-6 of the FSASEQUENCE algorithm initializes both the return FSA sequence $\left(\mathcal{A}_{r}^{n}\right)_{n=0}^{k}$ and the return factor sequence $\left(f_{r}^{n}\right)_{n=0}^{k}$ and then loops from 0 until $k$. Line 3 of the FSASEQUENCE algorithm uses the LENGTHFSA algorithm create a bounding FSA which only accepts strings of length $i$. The LenGTHFSA algorithm is shown in Figure 3.13b and accepts the length integer $k$ and the alphabet $\Sigma$ as parameters. Lines 2-4 of LENGTHFSA initialize the bounding FSA $\mathcal{A}$ with only the non-accepting start state $q_{0}$ which is then stored as the current state $q$. The loop on lines 5-11 of LENGTHFSA is used to create the bounding FSA which accepts only of length $k$ in the alphabet $\Sigma$. Line 6 of LENGTHFSA creates a new state $q_{n}$ and adds this state to the FSA set of states $Q \in \mathcal{A}$. The loop on lines 7-9 of LengthFSA creates a transition from the current state $q$ to the new state $q_{n}$ for every symbol $\alpha \in \Sigma$. On line 10 of LENGTHFSA the current state $q_{b}$ is updated as the new state $q_{n}$ for the next iteration of the loop. After the loop is completed, line 12 of LENGTHFSA adds the last newly added state $q_{n}$ to the set of accepting states $F \in \mathcal{A}$. Line 13 of LEnGthFSA returns the FSA $\mathcal{A}$ which is now the desired bounding FSA accepting all strings of length $k$ as $\mathcal{A}_{b}$ on Line 3 of FSASEquence. Line 4 of FSASEquence intersects the initial FSA $\mathcal{A}_{\text {init }}$ and the bounding FSA $\mathcal{A}_{b}$ producing the intersected FSA $\mathcal{A}_{r}^{i}$ at index $i$ in the FSA sequence $\left(\mathcal{A}_{r}^{n}\right)_{n=0}^{k}$. Line 5 of FSASEQUENCE sets the factor at index $i$ to 1 for the factor sequence $\left(f_{r}^{n}\right)_{n=0}^{k}$. The loop on lines 2-6 of FSASEQUENCE continues until all the FSAs in the FSA sequence $\left(\mathcal{A}_{r}^{n}\right)_{n=0}^{k}$ are created and all the factors in the factor sequence $\left(f_{r}^{n}\right)_{n=0}^{k}$ are set to 1 . Finally, line 7 of FSASEQUENCE returns both 
the completed FSA and factor sequences. These FSA and factor sequences are then added to the bounding length $k$ and the alphabet $\Sigma$ to complete the creation of an aggregate automata model.

\subsubsection{String Operations}

1: procedure $\mathrm{ABINARYOP}\left(\mathrm{OP}, \mathcal{M}_{1}, \mathcal{M}_{2}, \Sigma\right)$

2: $\quad\left(\mathcal{A}_{1}^{n}\right)_{n=0}^{k} \in \mathcal{M}_{1},\left(\mathcal{A}_{2}^{n}\right)_{n=0}^{k} \in \mathcal{M}_{2}$

3: $\quad \mathcal{A}_{U_{2}} \leftarrow \bigcup_{n=0}^{k} \mathcal{A}_{2}^{n}$

4: $\quad$ for $\mathcal{A}_{o p}^{i} \in\left(\mathcal{A}_{o p}^{n}\right)_{n=0}^{k}, f_{o p}^{i} \in\left(f_{o p}^{n}\right)_{n=0}^{k}$ do

5: $\quad \mathcal{A}_{o p}^{i}, f_{o p}^{i} \leftarrow \operatorname{OP}\left(\mathcal{A}_{1}^{i}, f_{1}^{n}, \mathcal{A}_{U_{2}}\right)$

6: $\quad$ end for

7: $\quad \mathcal{M}_{o p} \leftarrow\left\langle\left(\mathcal{A}_{o p}^{n}\right)_{n=0}^{k},\left(f_{o p}^{n}\right)_{n=0}^{k}, k, \Sigma\right\rangle$

8: $\quad$ return $\mathcal{M}_{o p}$

9: end procedure

(a) String operation and predicate coordination algorithm for two aggregate automata model arguments
: procedure RESTRUCTFSAs $\left(\mathcal{M}_{o p}\right)$

$\left(\mathcal{A}_{o p}^{n}\right)_{n=0}^{k} \in \mathcal{M},\left(f_{o p}^{n}\right)_{n=0}^{k} \in \mathcal{M}, \Sigma \in \mathcal{M}$

$k_{r} \leftarrow \operatorname{GetMaxLength~}\left(\left(\mathcal{A}_{n}\right)_{n=0}^{k}\right)$

for $\mathcal{A}_{r}^{i} \in\left(\mathcal{A}_{r}^{m}\right)_{m=0}^{k_{r}}, f_{r}^{i} \in\left(f_{r}^{m}\right)_{m=0}^{k_{r}}$ do

$f_{r}^{i} \leftarrow 0$

$\mathcal{A}_{b} \leftarrow \operatorname{LengthFSA}(i, \Sigma)$

for $\mathcal{A}_{I}^{j} \in\left(\mathcal{A}_{t}^{n}\right)_{n=0}^{k}$ do

$\mathcal{A}_{I}^{j} \leftarrow \mathcal{A}_{o p}^{j} \cap \mathcal{A}_{b}$

if $\mathcal{L}\left(\mathcal{A}_{I}^{j}\right) \neq \varnothing$ then

$f_{r}^{i} \leftarrow f_{r}^{i}+f_{i}$

end if

end for

$\mathcal{A}_{r}^{i} \leftarrow \bigcup_{n=0}^{k_{r}} \mathcal{A}_{I}^{n}$

end for

$\mathcal{M}_{r} \leftarrow\left\langle\left(\mathcal{A}_{r}^{n}\right)_{n=0}^{k_{r}},\left(f_{r}^{n}\right)_{n=0}^{k_{r}}, k_{r}, \Sigma\right\rangle$

return $\mathcal{M}_{r}$

17: end procedure

(b) Restructuring algorithm for aggregate automata models

Figure 3.14: Aggregate automata model utility algorithms for string operations and predicates

Since the aggregate automata model contains a sequence of FSAs instead of a single FSA, the simulation of string operations and predicates with more than one aggregate automata model argument must now manage how the operation or predicate will be simulated using two or more FSA sequences. The strategy we use in our work for these multiple FSA sequences is to merge each non-primary FSA sequence into a single FSA which can be used to simulate a string operation or predicate. Figure $3.14 \mathrm{a}$ is an algorithm for binary operations between two aggregate automata models 
which details this merging of non-primary FSA sequences and the subsequence string operation or predicate simulation. The ABINARYOP algorithm takes an operation or predicate simulation algorithm Op as a parameter in addition to two automata model parameters $\mathcal{M}_{1}$ and $\mathcal{M}_{2}$ and the alphabet $\Sigma$. Line 2 of the algorithm extracts the FSA sequences for both the $\mathcal{M}_{1}$ and $\mathcal{M}_{2}$ models for later use in the algorithm. Line 3 merges all the FSAs in the FSA sequence for $\mathcal{M}_{2}$ using the UNION operation and producing the merged FSA $\mathcal{A}_{U_{2}}$. Line 4 initializes the operation or predicate result FSA sequence $\left(\mathcal{A}_{o p}^{n}\right)_{n=0}^{k}$ and operation result factor sequence $\left(f_{o p}^{n}\right)_{n=0}^{k}$ before the loop on lines 4-6. Line 5 of this loop performs the Op operation or predicate simulation algorithm using the FSA and factor at index $i$ of their respective sequences for $\mathcal{M}_{1}$ and the merged FSA $\mathcal{A}_{U_{2}}$. The FSA $\mathcal{A}_{o p}^{i}$ and factor $f_{o p}^{i}$ produced by the Op algorithm are set as the respective FSA and factor at index $i$ in the result sequences $\left(\mathcal{A}_{o p}^{n}\right)_{n=0}^{k}$ and $\left(f_{o p}^{n}\right)_{n=0}^{k}$. Lines 7 and 8 finish the algorithm by creating the operation or predicate result aggregate automata model $\mathcal{M}_{o p}$ on line 7 and returning that model from the algorithm on line 8 .

Unfortunately, the UNION of non-primary FSA sequences for string operations and predicates can result in an aggregate automata model which is vulnerable to subtractive collapses due to produced FSA sequence containing individual FSAs which accept strings of different lengths. Since the aggregate model was created specifically for the prevention of subtractive collapses, this continuing vulnerability must be removed by restructuring the FSA sequence produced by the simulation of the string operation or predicate $\left(\mathcal{A}_{o p}^{n}\right)_{n=0}^{k}$. Figure $3.14 \mathrm{~b}$ shows the RESTRUCTFSAs algorithm which performs this restructuring and accepts the aggregate automata model $\mathcal{M}_{o p}$ as its only parameter. Line 2 of the algorithm extracts the FSA and factor sequences $\left(\mathcal{A}_{o p}^{n}\right)_{n=0}^{k}$ and $\left(f_{o p}^{n}\right)_{n=0}^{k}$ and the alphabet $\Sigma$ from the model $\mathcal{M}_{o p}$. Line 3 uses the 
GetMaxLength algorithm to determine the length $k_{r}$ longest accepted string in the FSA sequence $\left(\mathcal{A}_{n}\right)_{n=0}^{k}$. The loop on lines 4-14 begins by initializing the return FSA and factor sequences $\left(\mathcal{A}_{r}^{m}\right)_{m=0}^{k_{r}}$ and $\left(f_{r}^{m}\right)_{m=0}^{k_{r}}$ before iterating from 0 to $k_{r}$. Line 5 within the loop sets the factor $f_{r}^{i}$ of the sequence $\left(f_{r}^{m}\right)_{m=0}^{k_{r}}$ at index $i$ to 0 . On line 6, the algorithm LengthFSA seen in Figure 3.13b is used to create bounding FSA $\mathcal{A}_{b}$ accepting only strings of length $i$. The loop on lines 7-12 begins by initializing the FSA sequence $\left(\mathcal{A}_{I}^{n}\right)_{n=0}^{k}$ before iterating from 0 to $k$. Line 8 utlizes the bounding FSA created on line $6 \mathcal{A}_{b}$ to bound the FSA $\mathcal{A}_{o p}^{j}$ using the Intersection automata operation to produce the resulting FSA $\mathcal{A}_{I}^{j}$. Lines 9-11 are an if condition which is used to increment the factor $f_{r}^{i}$. After the completion of the loop from lines 7-12, all the intersected FSAs in the sequence $\left(\mathcal{A}_{I}^{n}\right)_{n=0}^{k}$ are merged using the UnION operation on line 13 to produce a single FSA $\mathcal{A}_{r}^{i}$ which accepts all strings with a length $i$ accepted by the sequence $\left(\mathcal{A}_{o p}^{n}\right)_{n=0}^{k}$. Due to the if condition on lines 9-11, the factor $f_{r}^{i}$ at the time line 13 is performed will equal the sum of all factors from the sequence $\left(f_{o p}^{n}\right)_{n=0}^{k}$ which correspond to FSAs in the sequence $\left(\mathcal{A}_{o p}^{n}\right)_{n=0}^{k}$ accepting strings of length $i$. This summation of factors is done to preserve the adjustments made to factors due to string operations and predicates expressed in the $\left(f_{o p}^{n}\right)_{n=0}^{k}$ sequence. Line 15 of the RESTRUCTFSAs algorithm creates a new aggregate automata model $\mathcal{M}_{r}$ from the sequence of merged FSAs $\left(\mathcal{A}_{r}^{n}\right)_{n=0}^{k_{r}}$, the sequence of summed factors $\left(f_{r}^{n}\right)_{n=0}^{k_{r}}$, the newly computed bounding length $k_{r}$, and the alphabet $\Sigma$. Line 16 finishes the algorithm by returning this newly created $\mathcal{M}_{r}$ model.

While this process of restructuring the FSA sequence does ensure that subtractive collapses do not occur, the additional performance costs are incurred due to the restructuring. Specifically, more time is required to complete operation and predicate simulations, additional temporary space is required to create the sequence of 
sequences, and the restructured sequence of FSAs becomes vulnerable to collapse due to the UNION of the bounded FSAs (this type of collapse is discussed in more detail in Section 3.3.4).

\subsubsection{Model Counting}

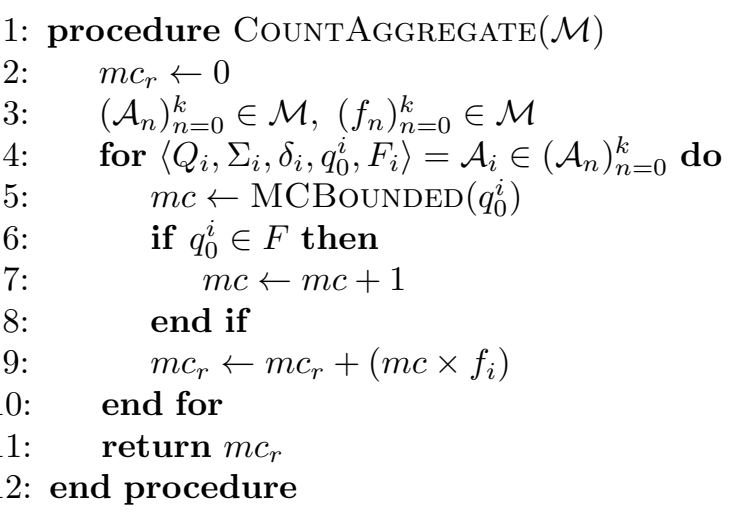

Figure 3.15: Coordinating algorithm aggregate automata models

The model counting algorithm for the aggregate automata model is shown in Figure 3.15 utilizing the recursive MCBOUNDED algorithm shown previously in Figure 3.8b and discussed in Section 3.2.1. The coordinating algorithm begins by initializing the return model count $m c_{r}$ as 0 . Next, the FSA sequence and the factor sequence are retrieved from the aggregate automata model on Lines 3 and 4. Lines 5-11 contain an iteration through each FSA quintuple $\left\langle Q_{i}, \Sigma_{i}, \delta_{i}, q_{0}^{i}, F_{i}\right\rangle=\mathcal{A}_{i} \in \mathcal{A}_{i}$ in the aggregate model $\mathcal{M}$. For each of these FSAs, the model count is determined with a call to the recursive algorithm MCBOUNDED and stored as the current model count $m c$ as shown on Line 6. Lines 7-9 account for an empty string with an if condition which checks if the current start state $q_{0}^{i}$ is accepting and increments the current model count $m c$. Line 9 is where the return model count $m c_{r}$ is updated by the addition of the current model count $m c$ multiplied by the factor $f_{i}$ corresponding to the current 
FSA $\mathcal{A}_{i}$. After iterating through all the FSAs in the aggregate automata model, the return model count $m c_{r}$ is returned from the algorithm.

\subsubsection{Over-Approximation}

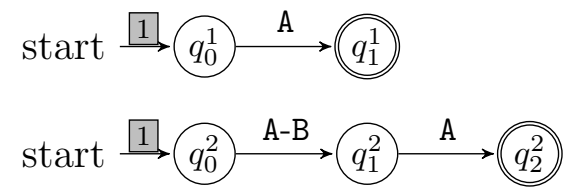

(a) $\mathcal{M}_{1}$ aggregate automata model

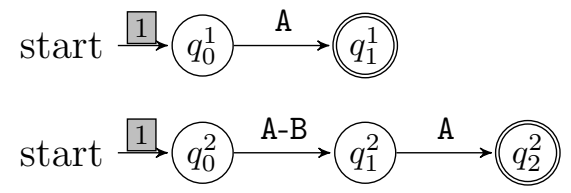

(b) $\mathcal{M}_{2}$ aggregate automata model

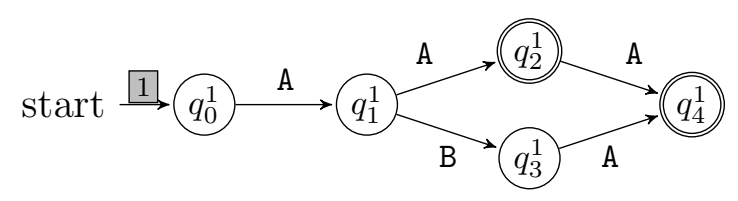

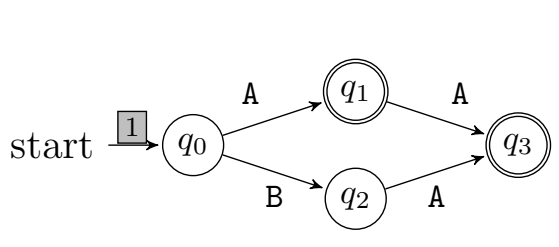

(c) $\mathcal{M}_{U_{2}}$ aggregate automata model

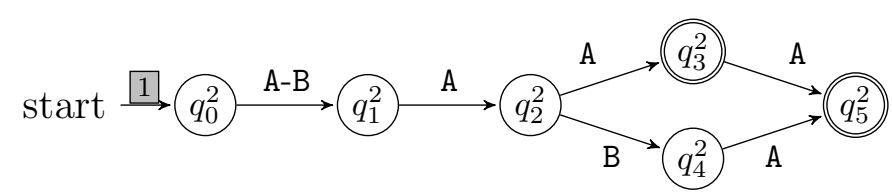

(d) $\mathcal{M}_{C}$ aggregate automata model resulting CONCATENATION operation

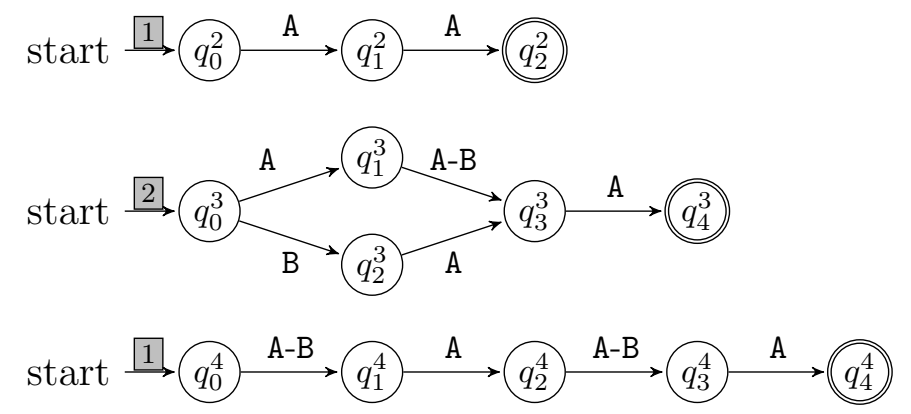

(e) $\mathcal{M}_{R}$ aggregate automata model after RESTRUCTFSAs

Figure 3.16: Concatenation $\left(\mathcal{M}_{1}, \mathcal{M}_{2}\right)$ example for aggregate automata model

The example shown in Figure 3.16 demonstrates how over-approximation due to model structure is prevented for aggregate automata models. This example presents the string operation CONCATENATION $\left(s t r_{1}, s t r_{2}\right)$ simulated for aggregate automata 
models $\mathcal{M}_{1}$ and $\mathcal{M}_{2}$ representing the string variables $s t r_{1}$ and $s t r_{2}$ respectively. Figures 3.16a and 3.16b show the diagrams corresponding to $\mathcal{M}_{1}$ and $\mathcal{M}_{2}$ such that $\mathcal{M}_{1}$ and $\mathcal{M}_{2}$ both have an alphabet $\Sigma_{\mathcal{M}_{1}}=\Sigma_{\mathcal{M}_{2}}=\{\mathrm{A}, \mathrm{B}\}$ and bounding length $k=2$ and both represent the solution set $\mathcal{S}_{\mathcal{M}_{1}}=\mathcal{S}_{\mathcal{M}_{2}}=\{\mathrm{A}, \mathrm{AA}, \mathrm{BA}\}$. Figure 3.16c shows the diagram of the aggregate automata model $\mathcal{M}_{U_{2}}$ which is produced on line 3 of the ABInARYOp procedure from the FSA sequence in the $\mathcal{M}_{2}$ model. Figure 3.16d shows the aggregate model $\mathcal{M}_{C}$ produced by simulating the ConCATEnATion operation for $\mathcal{M}_{1}$ and $\mathcal{M}_{U_{2}}$. Figure 3.16e shows the aggregate model $\mathcal{M}_{R}$ which was created by the RestruCtFSAs algorithm performed on the $\mathcal{M}_{C}$ model. While there are 2 additional intermediary steps in the aggregate version of this CoNCATENATiOn operation compared to the bounded version from Section 3.2.2, the aggregate automata model also does not over-approximate the solution set. The 8 values in the aggregate model's solution set match the expected solution set of 8 , i.e. $\mathcal{S}_{e}=\mathcal{S}_{\mathcal{M}_{R}}=\{$ AA, AAA, ABA, BAA, AAAA, AABA, BAAA, BABA $\}$. This provides an example of how over-approximation of a solution set due to an automata model is prevented in aggregate automata models.

While aggregate automata models avoid over-approximation of the solution set, the use of factors in the calculation of the model count as seen in the CountAGGREGATE algorithm in Figure 3.15 can result in over-approximation of the model count calculated for the model. The previous example in Figure 3.16 shows how this type of model count over-approximation can occur. The aggregate automata models $\mathcal{M}_{1}$ and $\mathcal{M}_{2}$, shown in figures 3.16a and 3.16a respectively, both have a model count $M C\left(\mathcal{M}_{1}\right)=M C\left(\mathcal{M}_{2}\right)=3$ which should produce an aggregate model from the Concatenation operation with a model count of 9. However, the model count of the restructured aggregate model $M C\left(\mathcal{M}_{R}\right)=11$. This over-approximation 
occurs due to the summation of the factors on lines 9-11 of the RESTRUCTFSAs algorithm in Figure 3.14b. When this condition on lines 9-11 occurs with an $i$ value of 3, the factors from $\mathcal{A}_{C}^{1}$ and $\mathcal{A}_{C}^{2}$ FSAs are summed to produce a factor of 2 for the $\mathcal{A}_{R}^{3}$. This new factor of 2 is used to reflect the merging both of the AAA string value from the $\mathcal{A}_{C}^{1}$ FSA and the AAA string value from the $\mathcal{A}_{C}^{2}$ FSA into the $\mathcal{A}_{R}^{3}$ FSA. However, since the the $\mathcal{A}_{R}^{3}$ FSA also represents ABA string value from $\mathcal{A}_{C}^{1}$ but not $\mathcal{A}_{C}^{2}$ and the BAA string value from $\mathcal{A}_{C}^{2}$ and not $\mathcal{A}_{C}^{1}$, both the ABA and BAA string values are over-approximated in the $\mathcal{A}_{R}^{3}$ FSA. It is due to this merging of FSAs and the corresponding summation of factors which results in model count over-approximation for aggregate automata models.

\subsubsection{Collapse}

While the aggregate automata model does improve upon the bounded model by preventing collapses due to subtractive string operations, aggregate automata models are still susceptible to collapses from substitutive string operations.

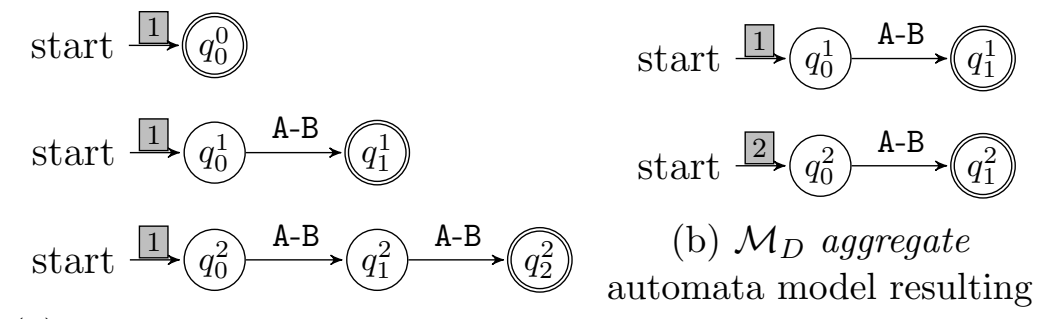

(a) $\mathcal{M}_{1}$ aggregate automata model from DELETE operation

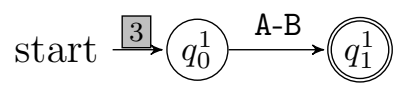

(c) $\mathcal{M}_{R}$ aggregate automata model after restructuring the FSA sequence

Figure 3.17: $\operatorname{DeLETE}\left(\mathcal{M}_{1}, 1,2\right)$ example for aggregate automata model

The example shown in Figure 3.17 demonstrates how the collapse problem is prevented for aggregate automata models due to subtractive string operations. This example presents the string operation $\operatorname{DeLETE}\left(s r_{1}\right.$, int $\left._{s}, i n t_{e}\right)$ simulated for the $a g$ gregate automata model $\mathcal{M}_{1}$ and the start and end indices 1 and 2. Figure 3.17a shows 
the diagram corresponding to $\mathcal{M}_{1}$ such that $\mathcal{M}_{1}$ has an alphabet $\Sigma_{\mathcal{M}_{1}}=\{\mathrm{A}, \mathrm{B}\}$ and maximum length $k=2$ and represents the solution set $\mathcal{S}_{\mathcal{M}_{1}}=\{\varepsilon, \mathrm{A}, \mathrm{B}, \mathrm{AA}, \mathrm{AB}, \mathrm{BA}, \mathrm{BB}\}$. Figure $3.17 \mathrm{~b}$ shows the diagram of the aggregate automata model $\mathcal{M}_{D}$ which is returned from the $\operatorname{DeLETE}\left(\mathcal{M}_{1}, 1,2\right)$ operation and has the same alphabet as $\mathcal{M}_{1}$, $\Sigma_{\mathcal{M}_{D}}=\{A, B\}$. The prevention of the collapse due to the DeLETE operation can be seen by comparing the $\mathcal{M}_{1}$ model count $M C\left(\mathcal{M}_{1}\right)=7$ and the $\mathcal{M}_{D}$ model count $M C\left(\mathcal{M}_{D}\right)=6$ which is the expected outcome due to the empty string $\varepsilon$ not being a valid argument for the DELETE operation. This prevention of subtractive collapse is accomplished first by the DELETE algorithm which preserves the 4 distinct string values $\mathrm{AA}, \mathrm{AB}, \mathrm{BA}$, and $\mathrm{BB}$ from the FSA $\mathcal{A}_{1}^{2}$ as the 2 distinct string values $\mathrm{A}$ and B accepted by the FSA $\mathcal{A}_{D}^{2}$ and the factor $f_{D}^{2}=2$. The 6 expected distinct string values represented by $\mathcal{M}_{D}$ are maintained in $\mathcal{M}_{R}$ produced from the RESTRUCTFSAs algorithm with the model count $M C\left(\mathcal{M}_{R}\right)=6$. This example demonstrates why the factor summation on lines 9-11 of the RESTRUCTFSAs algorithm is necessary to prevent subtractive collapses where the merging of the FSAs $\mathcal{A}_{D}^{1}$ and $\mathcal{A}_{D}^{2}$ requires the summation of their respective factors $f_{D}^{1}=1$ and $f_{D}^{2}=2$ to preserver the model count $M C\left(\mathcal{M}_{R}\right)=6$ calculated from the 2 string values accepted by $\mathcal{A}_{R}^{1}$ multiplied by the factor $f_{R}^{1}=3$. It is this use of an FSA sequence and summation of factors which allows aggregate automata models to prevent collapse problems due to subtractive string operations.

The example shown in Figure 3.18 demonstrates how the collapse problem can occur for aggregate automata models due to substitutive string operations. This example presents the string operation REPLACE $\left(\operatorname{str}_{1}\right.$, char $_{1}$, char $\left._{2}\right)$ simulated for the aggregate automata model $\mathcal{M}_{1}$ representing the string argument $s t r_{1}$ and the symbols A and B as the symbol arguments char ${ }_{1}$ and char $_{2}$. Figure 3.18a shows the diagram 


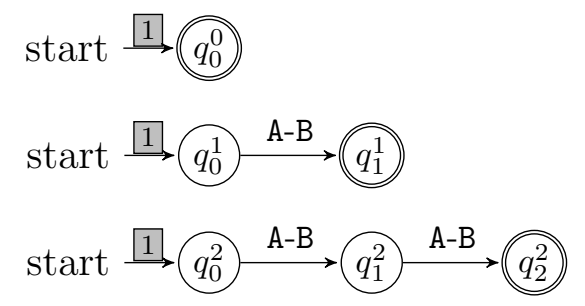

(a) $\mathcal{M}_{1}$ aggregate automata model

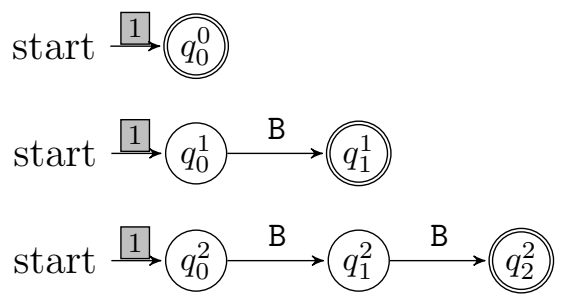

(b) $\mathcal{M}_{R}$ aggregate automata model resulting from REPLACE operation

Figure 3.18: $\operatorname{RePLACE}\left(\mathcal{M}_{1}, \mathrm{~A}, \mathrm{~B}\right)$ example for aggregate automata model

corresponding to $\mathcal{M}_{1}$ such that $\mathcal{M}_{1}$ has an alphabet $\Sigma_{\mathcal{M}_{1}}=\{\mathrm{A}, \mathrm{B}\}$ and maximum length $k=2$ and represents the solution set $\mathcal{S}_{\mathcal{M}_{1}}=\{\varepsilon, \mathrm{A}, \mathrm{B}, \mathrm{AA}, \mathrm{AB}, \mathrm{BA}, \mathrm{BB}\}$. Figure $3.18 \mathrm{~b}$ shows the diagram of the aggregate automata model $\mathcal{M}_{R}$ which is returned from the $\operatorname{ReplaCE}\left(\mathcal{M}_{1}, \mathrm{~A}, \mathrm{~B}\right)$ operation and has the same alphabet as $\mathcal{M}_{1}, \Sigma_{\mathcal{M}_{R}}=\{\mathrm{A}, \mathrm{B}\}$. There is no need to use the ReStRUCtreFSAs procedure on $\mathcal{M}_{R}$ because simple substitutive operations do not alter the length of the strings accepted by FSAs which is the reason such a procedure is required. It is clear that the 7 distinct string values represented in $\mathcal{M}_{1}$ by the model count $M C\left(\mathcal{M}_{1}\right)=7$ are not properly represented in the $\mathcal{M}_{R}$ model with the model count $M C\left(\mathcal{M}_{R}\right)=3$. While the empty string $\varepsilon$ is properly represented in both the $\mathcal{A}_{1}^{0}$ and $\mathcal{A}_{R}^{0}$ models, the A and B string values in $\mathcal{A}_{1}^{1}$ are only represented as the single value $\mathrm{B}$ in $\mathcal{A}_{R}^{1}$ and the $\mathrm{AA}, \mathrm{AB}, \mathrm{BA}$, and $\mathrm{BB}$ string values in $\mathcal{A}_{1}^{2}$ are only represented by the single string value BB in $\mathcal{A}_{R}^{2}$. It is in this way that collapses due to substitutive string operations can occur for aggregate automata models. 


\subsection{Weighted Transition Automata Model}

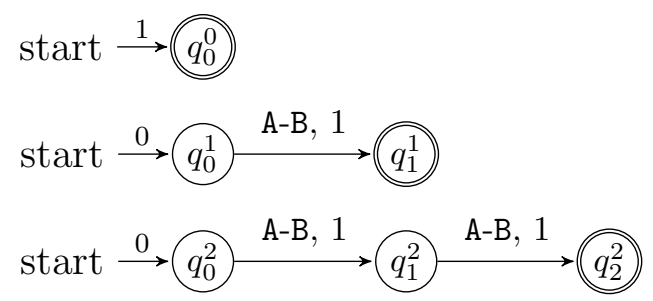

Figure 3.19: Weighted-Transition Aggregate Bounded Automata Model

The weighted-transition aggregate automata model, shortened henceforth as weighted automata model, was created primarily to prevent collapse problems which occur due to substitutive string operations as described in Sections 3.1.3, 3.2.3, and 3.3.4. The weighted automata model was also created with the intent of preventing the model count over-approximation and UNION collapses observed in aggregate automata models. Figure 3.19 is an example of a weighted automata model with an alphabet $\Sigma=\{\mathrm{A}, \mathrm{B}\}$ and a length value $k=2$. As the example shows, the weighted automata model is nearly identical to the aggregate automata model due to the weighted model building upon the improvements made in the aggregate model for preventing subtractive collapse. In fact, there are only two differences between the two automata models: the weighted model uses a weighted-transition finite state automaton (WFSA) sequence instead of the FSA sequence used in the aggregate model and the lack of a factor sequence in the weighted model. The factor sequence of the aggregate model is incorporated as transition weights of the new WFSAs which prevents the model count over-approximation in the weighted model. Before detailing the creation process for a weighted automata model in Section 3.4.2, the new type of automaton, the WFSA, will be detailed in Section 3.4.1. 


\subsubsection{Weighted-Transition Finite State Automaton}

A weighted-transition finite state automaton (WFSA) is a modification of FSA so that in addition to accepting or rejecting input strings, the actual number of identical input strings accepted can be determined. Due to this ability to quantify accepted input strings, the language accepted by the WFSA $\mathcal{W}$, called the language of the WFSA and denoted as $\mathcal{L}(\mathcal{W})$, is not a set of strings but is instead a multiset or bag of strings. Because the language of the WFSA is a multiset rather than a set of strings, a WFSA can accept and therefore represent multiple instances of the same input string. More formally, a weighted-transition finite state automaton $\mathcal{W}$ is defined as the 6 -tuple $\left(Q, \Sigma, \delta, q_{0}, F, i\right)$ where

- $Q$ is a finite set of states, $Q \neq \varnothing$

- $\Sigma$ is a finite set of symbols called the alphabet, $\Sigma \neq \varnothing$

- $\delta$ is the transition function, $\delta: Q \times \Sigma \times \mathbb{Z} \rightarrow Q$

- $q_{0}$ is the start state, $q_{0} \in Q$

- $F$ is the set of accepting or final states, $F \subseteq Q$

- $i_{\varepsilon}$ is an integer counting the number of empty strings represented by the start state $q_{0}$ if the start state is an accepting state $q_{0} \in F$

There are two notable differences between the FSA quintuple and the WFSA 6-tuple: the addition of the empty string counter $i_{\varepsilon}$ and the added positive integer argument in the transition function. The addition of the empty string counter allow a WFSA to represent more than a single empty string value which can be required due to either a subtractive or a complex substitutive string operation. For example, the string operation $\operatorname{DeLETE}(s t r, 0,1)$ could create one or more empty strings when deleting strings of length 1, but would only be represented in an FSA by a single accepting start 
state. Since a WFSA can represent more than a single empty string via the empty string counter $i_{\varepsilon}$, the DELETE operation can be represented accurately. The transition function of a WFSA $\delta_{W F S A}:\left(q, \alpha, i_{w}\right) \rightarrow q_{d}$ differs from the transition function of a FSA $\delta_{W F S A}:(q, \alpha) \rightarrow q_{d}$ due to the addition of the $i_{w}$ positive integer weight parameter. It is this weight parameter in the WFSA transition function that allows a WFSA to accept a multiset of string values rather than just a set. Since collapse problems for symbolic string models occur due to using set based models to reprent multisets of string values in quantitative analyses, the ability of WFSAs to accept a multiset of strings is essential to solving collapse problems for automata-based symbolic string models.

In addition to these differences in the WFSA 6-tuple compared to the FSA quintuple, the automata operation for the WFSA must be changed from their FSA counterparts. Specifically, the minimization algorithm (whether Hopcroft, Moore, or Brzozowski), SubsetConstruction (determinization), Intersection, Union, Subtraction, Concatenation, and Complement automata operations must be modified so that they perform appropriately with the new weighted transitions $\delta:\left(q, \alpha, i_{w}\right) \rightarrow q_{d}$ and empty string counter $i_{\varepsilon}$. In particular, the SubsetConSTRUCTION and minimization algorithms must ensure that transitions are duplicates according to states, symbols, and weights before reducing such duplicate transitions to single transitions with increased weights.

\subsubsection{Model Creation}

The creation process for WFSA sequence of the weighted model is shown in Figure 3.20 and starts with the WFSASEQUENCE algorithm shown in Figure 3.20a. WFSASEQUENCE requires an initial WFSA $\mathcal{W}_{\text {init }}$ similar to the five initial FSAs 


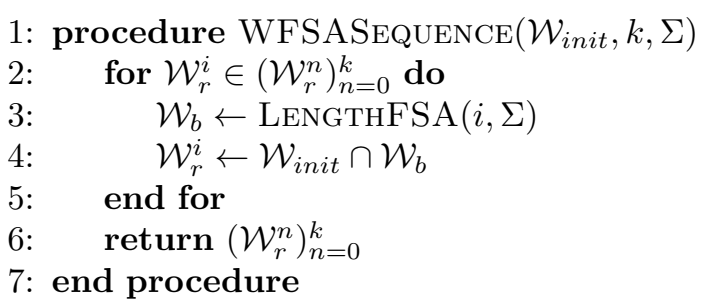

(a) Creation algorithm for FSA sequence

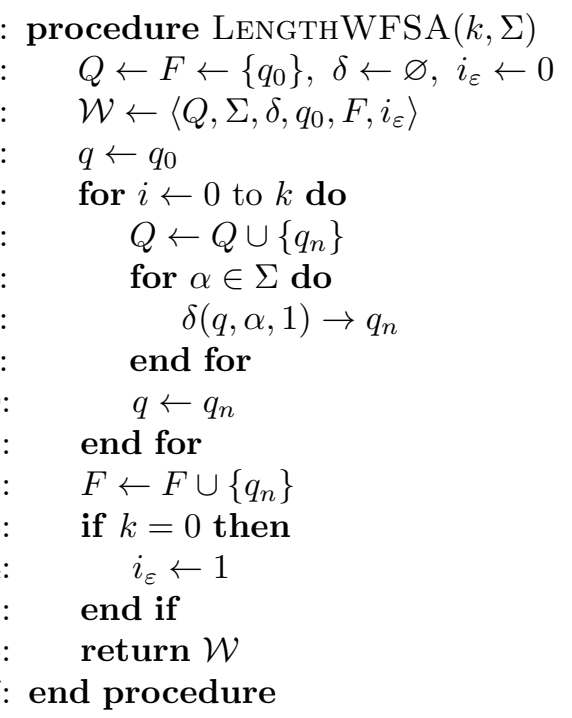

(b) Creation algorithm for length bounded FSA

Figure 3.20: Algorithms to create WFSA sequence for weighted automata model

described in Section 2.4.2: empty, empty string, literal, simple unknown, and complex unknown. Also required by the WFSASEQUENCE procedure are an initial bounding length $k$ and an alphabet $\Sigma$. WFSASEQUENCE begins by initializing the WFSA sequence $\left(\mathcal{W}_{r}^{n}\right)_{n=0}^{k}$ on line 2 before iterating the loop on lines $2-5$ from 0 to $k$. Each iteration of the loop creates a bounding WFSA on line 3 using the LENGTHWFSA algorithm with the current $i$ value and the alphabet $\Sigma$. Similar to the LEngthFSA algorithm for aggregate automata models, the LENGTHWFSA algorithm shown in Figure $3.20 \mathrm{~b}$ is used to create a WFSA which accepts all strings of the specified length $k$ using every combination of symbols in the alphabet $\Sigma$. LENGTHWFSA initializes the return WFSA on lines 2-3 with the single non-accepting start state $q_{0}$, not transitions, and an initial empty string count $i_{\varepsilon}=0$. Line 4 of LENGTHWFSA sets the current state $q$ as the WFSA $\mathcal{W}$ start state $q_{0}$. Next in the LengthWFSA algorithm, the loop on lines 5-11 creates the structure of the bounding WFSA $\mathcal{W}$ 
while iterating from 0 to $k$. Line 6 within the loop creates a new state $q_{n}$ and adds it to the set of states $Q \in \mathcal{W}$. Lines 7-9 of LENGTHWFSA then create a transition from the current state $q$ to the new non-accepting state $q_{n}$ with a weight of 1 for every symbol in the specified alphabet $\Sigma$. The last part of this loop updates the current state $q$ by setting it to the newly added state $q_{n}$ allowing future loop iterations to add transitions from that state. After the loop in LengthWFSA used to create the WFSA structure, line 12 adds the current state $q$ to the set of accepting states $F \in \mathcal{W}$ making it the single accepting state in the WFSA. The if condition on lines 13-15 of LENGTHWFSA checks if $k$ is 0 and if so updates the empty string counter $i_{\varepsilon}$ to 1 . LenGthWFSA completes on line 16 by returning the created length bounding WFSA $W$ from the algorithm. WFSASEQUENCE resumes its iteration using the new bounding WFSA $W_{b}$ from line 3 in the INTERSECTION operation on line 4 between it the initial WFSA $\mathcal{W}_{\text {init }}$ producing a WFSA stored as $\mathcal{W}_{r}^{i}$ at index $i$ in the WFSA sequence $\left(\mathcal{W}_{r}^{n}\right)_{n=0}^{k}$. After WFSASEQUEnce finishes the loop from lines 2-5, the WFSA sequence $\left(\mathcal{W}_{r}^{n}\right)_{n=0}^{k}$ which is now complete is returned on line 6 . This returned WFSA sequence is used along with the bounding length $k$ and the alphabet $\Sigma$ to create the new weighted automata model.

\subsubsection{Model Counting}

Figure 3.21 shows the model counting algorithm for the weighted automata model. The coordinating algorithm CounTEWEIGHTED shown in 3.21a utilizes the recursive model counting algorithm MCWEIGHTED which is shown in 3.21b. The model counting process begins using the COUNTWEIGHTED procedure which accepts the weighted automata model $\mathcal{M}$ as its only parameter. CountWeighted starts by initializing the result model count $m c_{r}$ as 0 on line 2 and extracting the WFSA 
1: procedure CountWeighted $(\mathcal{M})$

$m c_{r} \leftarrow 0$

$\left(\mathcal{W}_{n}\right)_{n=0}^{k} \in \mathcal{M}$

for $\left\langle Q_{i}, \Sigma_{i}, \delta_{i}, q_{0}^{i}, F_{i}, i_{\varepsilon}^{i}\right\rangle \in\left(\mathcal{W}_{n}\right)_{n=0}^{k}$ do $m c_{r} \leftarrow m c_{r}+\operatorname{MCWeighted}\left(q_{0}, 1\right)$

if $q_{0}^{i} \in F_{i}$ then

$m c_{r} \leftarrow m c_{r}+i_{\varepsilon}^{i}$

end if

end for

return $m c_{r}$

end procedure

(a) Coordinating algorithm
1: procedure MCWeighted $(q, i)$

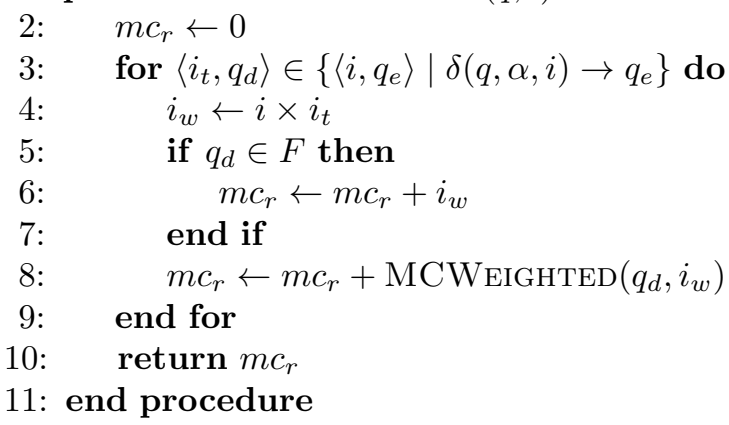

(b) Recursive model counting algorithm

Figure 3.21: Model counting algorithms for weighted automata models

sequence $\left(\mathcal{W}_{n}\right)_{n=0}^{k}$ from the $\mathcal{M}$ model on line 3 . The loop on lines 4-9 of CounTWEIGHTED are responsible for calculating the model count for each WFSA in the $\left(\mathcal{W}_{n}\right)_{n=0}^{k}$ sequence. Line 4 of COUnTWeighted begins the loop by extracting the WFSA 6-tuple $\left\langle Q_{i}, \Sigma_{i}, \delta_{i}, q_{0}^{i}, F_{i}, i_{\varepsilon}^{i}\right\rangle$ at the corresponding index $i$ for use in each iteration of the loop. Line 5 of CountWeighted utilizes the MCWeighted recursive algorithm with the start state $q_{0}^{i} \in \mathcal{W}_{i}$ and initial transition weight 1 as parameters to count the number of string values represented by WFSA $\mathcal{W}_{i}$. The MCWEIGHTED recursive algorithm takes two parameters, a WFSA state $q$ and an initial transition weight $i$. MCWEIGHTED begins by initializing its return model count $m c_{r}$ as 0 . The MCWEIGHTED procedure then loops through lines 3-9 for each transition $\delta(q, \alpha, i) \rightarrow q_{e}$ leaving the WFSA state $q$ using weight $i$ and destination state $q_{e}$ of the transition as $i_{t}$ and $q_{d}$ for each iteration of the loop. Line 4 of this loop multiplies the transition weight parameter $i$ by the weight $i_{t}$ for the loop iteration's transition to produce a new transition weight $i_{w}$. Lines 5-7 of MCWEIGHTED check if the destination state $q_{d}$ of the loop iteration's transition is an accepting state and if so the the return model count $m c_{r}$ is incremented by the new transition weight $i_{w}$. Line 8 of MCWEIGHTED then makes the recursive call to itself specifying the 
destination state $q_{d}$ of loop iteration's transition and the new transition weight $i_{w}$. After the loop in MCWEIGHTED is complete, line 10 returns the updated return model count $m c_{r}$. After CountWeighted increments the return model count $m c_{r}$ by model count returned from MCWeighted, lines 6-8 of CountWeighted ensure that the correct number of empty string values are accounted for by checking if $q_{0}^{i}$ is an accepting state and incrementing the return model count $m c_{r}$ by the empty string counter $i_{\varepsilon}^{i}$ if it is an accepting state. After CountWeighted completes the iteration through the loop on lines 4-9, the computed model count $m c_{r}$ is returned on line 10 . While this process of model counting weighted automata models is very similar to the same process for aggregate models, the use of WFSA does alter alter the model counting process. This alteration is most noticeable on line 6 of MCWEIGHTED which uses the newly computed transition weight $i_{w}$ to increment the model count instead of an increment of 1 per transition as is the case when model counting unbounded, bounded, and aggregate automata models.

\subsubsection{String Operations and Predicates}

The use of WFSAs for the weighted automata model requires the creation of additional string operation and predicate simulation algorithms since the existing FSA algorithms can not be used. These WFSA operation and predicate simulation algorithms are altered from their existing FSA versions in three primary ways: utilizing the WFSA specific automata operation algorithms, including the empty string counter, and adjusting WFSA transitions to incorporate weight adjustments. The use of the WFSA specific auotmata operation such as UNION and INTERSECTION is a very simple change needed since the WFSAs is used instead of FSAs. The inclusion of the empty string counter for adjustments is needed primarily for subtractive and complex 
substitutive operations which can produce an empty string value corresponding to more than one string values prior to the operation. The adjustments of transition weights by the simulation algorithms is is the means by which collapse problems are prevented in weighted automata models. Each of these three alterations of existing FSA operation and predicate simulation algorithms allows the performance of these algorithms to be compared between the weighted model and each of the unbound, bounded, and aggregate models with a focus on model differences rather than algorithm differences.

1: procedure WBINARYOp $\left(\mathrm{OP}, \mathcal{M}_{1}, \mathcal{M}_{2}, \Sigma\right)$

2: $\quad\left(\mathcal{W}_{1}^{n}\right)_{n=0}^{k} \in \mathcal{M}_{1},\left(\mathcal{W}_{2}^{n}\right)_{n=0}^{k} \in \mathcal{M}_{2}$

3: $\quad \mathcal{W}_{U_{2}} \leftarrow \bigcup_{n=0}^{k} \mathcal{W}_{2}^{n}$

4: $\quad$ for $\mathcal{W}_{o p}^{i} \in\left(\mathcal{W}_{o p}^{n}\right)_{n=0}^{k}$ do

5: $\quad \mathcal{W}_{o p}^{i} \leftarrow \operatorname{OP}\left(\mathcal{W}_{1}^{i}, \mathcal{W}_{U_{2}}\right)$

6: end for

7: $\quad \mathcal{M}_{o p} \leftarrow\left\langle\left(\mathcal{W}_{o p}^{n}\right)_{n=0}^{k}, k, \Sigma\right\rangle$

8: $\quad$ return $\mathcal{M}_{o p}$

9: end procedure

(a) String operation and predicate coordination algorithm for two weighted automata model arguments
1: procedure RESTRUCTWFSAs $\left(\mathcal{M}_{o p}\right)$

2: $\quad\left(\mathcal{W}_{o p}^{n}\right)_{n=0}^{k} \in \mathcal{M}, \Sigma \in \mathcal{M}$

3: $\quad k_{r} \leftarrow$ GetMaxLength $\left(\left(\mathcal{W}_{n}\right)_{n=0}^{k}\right)$

4: $\quad$ for $\mathcal{W}_{r}^{i} \in\left(\mathcal{W}_{r}^{m}\right)_{m=0}^{k_{r}}$ do

5: $\quad \mathcal{W}_{b} \leftarrow \operatorname{LEnGTHFSA}(i, \Sigma)$

6: $\quad$ for $\mathcal{W}_{I}^{j} \in\left(\mathcal{W}_{t}^{n}\right)_{n=0}^{k}$ do

7: $\quad \mathcal{W}_{I}^{j} \leftarrow \mathcal{W}_{o p}^{j} \cap \mathcal{W}_{b}$

8: $\quad$ end for

9: $\quad \mathcal{W}_{r}^{i} \leftarrow \bigcup_{n=0}^{k_{r}} \mathcal{W}_{I}^{n}$

10: $\quad$ end for

11: $\quad \mathcal{M}_{r} \leftarrow\left\langle\left(\mathcal{W}_{r}^{n}\right)_{n=0}^{k_{r}}, k_{r}, \Sigma\right\rangle$

12: $\quad$ return $\mathcal{M}_{r}$

13: end procedure

(b) Restructuring algorithm for weighted automata models

Figure 3.22: Weighted automata model utility algorithms for string operations and predicates

Since the weighted automata model uses a WFSA sequence in the same way that the aggregate model uses its FSA sequence, the same problems expressed in Section 3.3.1 for operations and predicate simulation are also problems that need addressing for weighted automata models. Figure 3.22 contains the WBINARYOP procedure in Figure 3.22a and the RestructWFSAs procedure in Figure 3.22b. The WBINARYOP procedure is used to coordinate the simulation of string opera- 
tions or predicates which require two weighted automata models. The WBINARYOP procedure functions very similarly to the ABINARYOP procedure where the only differences in the WBINARYOP version are the use of WFSAs instead of FSAs and the removal of factors. The RESTRUCTWFSAs procedure also functions very similarly to the RESTRUCTFSAs procedure for aggregate models where the only differences in the RESTRUCTWFSAs procedure for weighted models is the use of WFSAs instead of FSAs and the removal of factors. While the restructuring process for the WFSA sequence of a weighted automata model should not be nessesary to prevent subtractive collapses, the similar RESTRUCTWFSAs procedure is used to preserve consistency between the two models.

\subsubsection{Over-Approximation}

The example shown in Figure 3.23 demonstrates how over-approximation due to model structure is prevented for weighted automata models. This example presents the string operation CONCATENATION $\left(s t r_{1}, s t r_{2}\right)$ simulated for weighted automata models $\mathcal{M}_{1}$ and $\mathcal{M}_{2}$ representing the string variables $s t r_{1}$ and $s t r_{2}$ respectively. Figures $3.23 \mathrm{a}$ and $3.23 \mathrm{~b}$ show the diagrams corresponding to $\mathcal{M}_{1}$ and $\mathcal{M}_{2}$ such that $\mathcal{M}_{1}$ and $\mathcal{M}_{2}$ both have an alphabet $\Sigma_{\mathcal{M}_{1}}=\Sigma_{\mathcal{M}_{2}}=\{\mathrm{A}, \mathrm{B}\}$ and bounding length $k=2$

and both represent the solution set $\mathcal{S}_{\mathcal{M}_{1}}=\mathcal{S}_{\mathcal{M}_{2}}=\{\mathrm{A}, \mathrm{AA}, \mathrm{BA}\}$. Figure 3.23c shows the diagram of the weighted automata model $\mathcal{M}_{U_{2}}$ which is produced on line 3 of the WBInARYOP procedure from the WFSA sequence in the $\mathcal{M}_{2}$ model. Figure 3.23d shows the weighted automata model $\mathcal{M}_{C}$ produced by simulating the ConcAtenATION algorithm for $\mathcal{M}_{1}$ and $\mathcal{M}_{U_{2}}$. Figure $3.23 \mathrm{e}$ shows the weighted automata model returned from the RESTRUCTWFSAs procedure performed on the $\mathcal{M}_{C}$ model. Just as with the bounded and aggregate automata models, the weighted model prevents the 


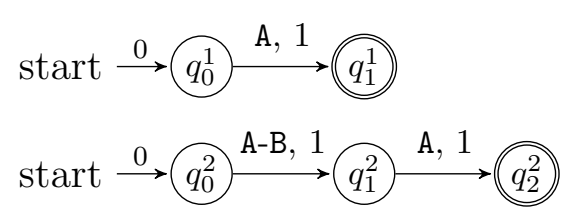

(a) $\mathcal{M}_{1}$ weighted automata model

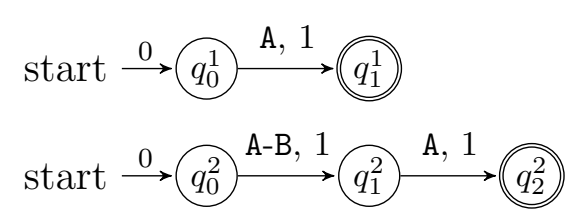

(b) $\mathcal{M}_{2}$ weighted automata model

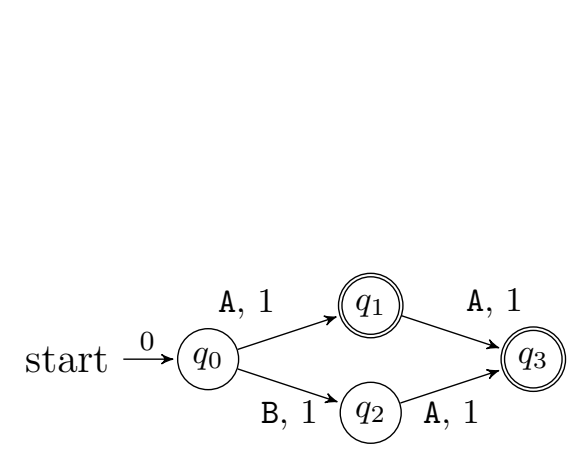

(c) $\mathcal{M}_{U_{2}}$ weighted automata model
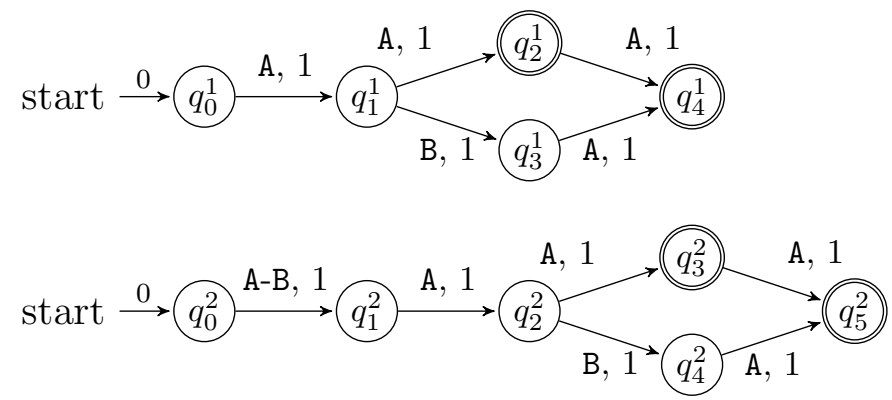

(d) $\mathcal{M}_{C}$ weighted automata model resulting Concatenation operation

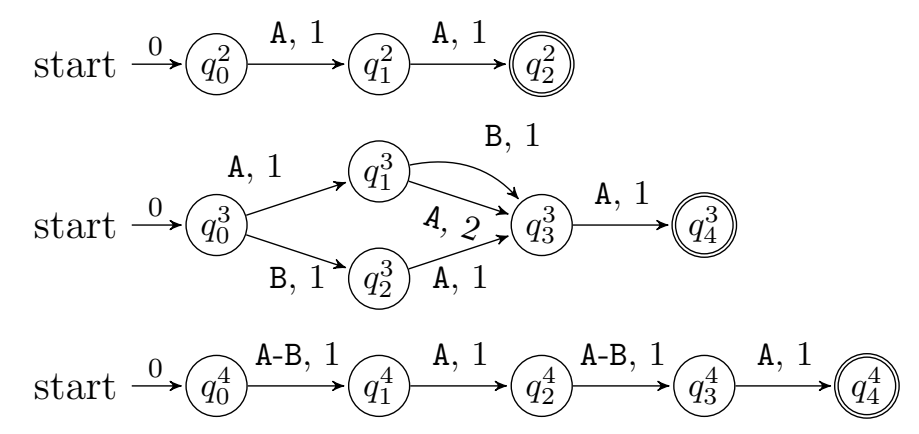

(e) $\mathcal{M}_{r}$ weighted automata model after restructuring the WFSA sequence

Figure 3.23: Concatenation $\left(\mathcal{M}_{1}, \mathcal{M}_{2}\right)$ example for weighted automata model

over-approximation of its solution set for the $\operatorname{Concatenation}\left(\mathcal{M}_{1}, \mathcal{M}_{2}\right)$ operation since the expected solution set $\mathcal{S}_{e}$ and the solution set of the restructured model $\mathcal{S}_{\mathcal{M}_{R}}$ are equal, i.e. $\mathcal{S}_{e}=\mathcal{S}_{\mathcal{M}_{R}}\{\mathrm{AA}, \mathrm{AAA}, \mathrm{ABA}, \mathrm{BAA}, \mathrm{AAAA}, \mathrm{AABA}, \mathrm{BAAA}, \mathrm{BABA}\}$. This example demonstrates how over-approximation of a solution set due to an automata model is prevented in weighted automata models.

The example in Figure 3.23 also demonstrates that weighted automata models do not suffer from model count over-approximation observed in aggregate models as 
described in Section 3.3.3. The expected model count is 9 for both the model produced by the Concatenation $\mathcal{M}_{C}$ and the restructured model $\mathcal{M}_{R}$ which matches the actual model counts $M C\left(\mathcal{M}_{C}\right)=M C\left(\mathcal{M}_{R}\right)=9$. Weighted automata models do not suffer from model count over-approximation because the factor sequence in aggregate models which is the cause for the over-approximation is not present in weighted models.

\subsubsection{Collapse}

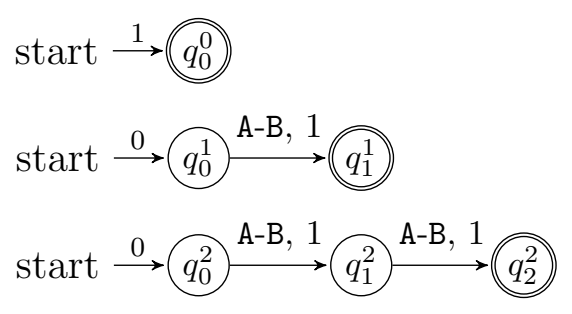

(a) $\mathcal{M}_{1}$ weighted automata model

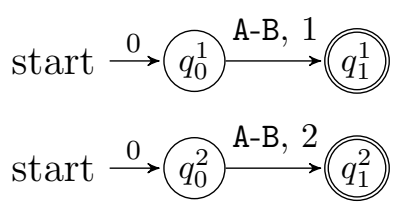

(b) $\mathcal{M}_{D}$ weighted automata model resulting from DELETE operation

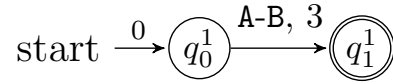

(c) $\mathcal{M}_{r}$ weighted automata model after restructuring the WFSA sequence

Figure 3.24: $\operatorname{DeLetE}\left(\mathcal{M}_{1}, 1,2\right)$ example for weighted automata model

The example shown in Figure 3.24 demonstrates how the collapse problem is prevented for weighted automata models due to subtractive string operations. This example presents the string operation $\operatorname{DELETE}\left(s t r_{1}, i n t_{s}, i n t_{e}\right)$ simulated for the weighted automata model $\mathcal{M}_{1}$ and the start and end indices 1 and 2. Figure 3.24a shows the diagram corresponding to $\mathcal{M}_{1}$ such that $\mathcal{M}_{1}$ has an alphabet $\Sigma_{\mathcal{M}_{1}}=\{A, B\}$ and maximum length $k=2$ and represents the solution set $\mathcal{S}_{\mathcal{M}_{1}}=\{\varepsilon, \mathrm{A}, \mathrm{B}, \mathrm{AA}, \mathrm{AB}, \mathrm{BA}, \mathrm{BB}\}$. Figure $3.24 \mathrm{~b}$ shows the diagram of the weighted automata model $\mathcal{M}_{D}$ which is returned from the $\operatorname{DELETE}\left(\mathcal{M}_{1}, 1,2\right)$ operation and has the same alphabet as $\mathcal{M}_{1}$, $\Sigma_{\mathcal{M}_{D}}=\{\mathrm{A}, \mathrm{B}\}$. The prevention of a collapse due to the DeLETE operation can be seen by comparing the expected model count of 6 (empty string is not a valid target of the $\operatorname{DELETE}\left(s t r_{1}, i n t_{s}, i n t_{e}\right)$ operation) to the model counts of both the model returned 
from the delete operation $M C\left(\mathcal{M}_{D}\right)=6$ and the restructured model $M C\left(\mathcal{M}_{R}\right)=6$. This is an example of the prevention of collapse due to subtractive operations using weighted automata models.

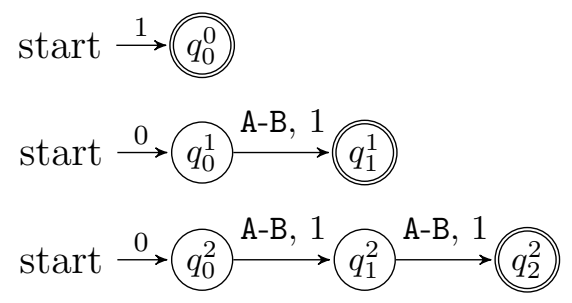

(a) $\mathcal{M}_{1}$ weighted automata model

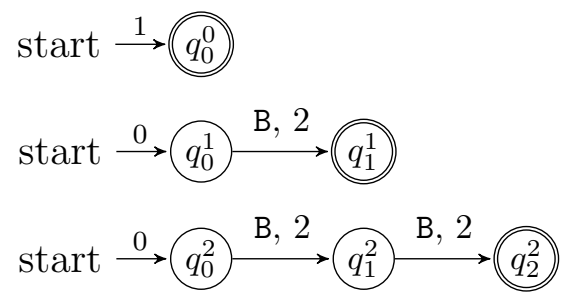

(b) $\mathcal{M}_{R}$ weighted automata model resulting from REPLACE operation

Figure 3.25: $\operatorname{ReplaCE}\left(\mathcal{M}_{1}, \mathrm{~A}, \mathrm{~B}\right)$ example for weighted automata model

The example shown in Figure 3.25 demonstrates how the collapse problem is prevented for weighted automata models due to substitutive string operations. This example presents the string operation $\operatorname{REPLACE}\left(s t r_{1}, \operatorname{char}_{1}, \operatorname{char}_{2}\right)$ simulated for the weighted automata model $\mathcal{M}_{1}$ representing the string argument $s t r_{1}$ and the symbols $\mathrm{A}$ and $\mathrm{B}$ as the symbol arguments $\operatorname{char}_{1}$ and $\operatorname{char}_{2}$. Figure 3.25a shows the diagram corresponding to $\mathcal{M}_{1}$ such that $\mathcal{M}_{1}$ has an alphabet $\Sigma_{\mathcal{M}_{1}}=\{\mathrm{A}, \mathrm{B}\}$ and maximum length $k=2$ and represents the solution set $\mathcal{S}_{\mathcal{M}_{1}}=\{\varepsilon, \mathrm{A}, \mathrm{B}, \mathrm{AA}, \mathrm{AB}, \mathrm{BA}, \mathrm{BB}\}$. Figure $3.25 \mathrm{~b}$ shows the diagram of the weighted automata model $\mathcal{M}_{R}$ which is returned from the $\operatorname{DeLete}\left(\mathcal{M}_{1}, 1,2\right)$ operation and has the same alphabet as $\mathcal{M}_{1}, \Sigma_{\mathcal{M}_{R}}=\{\mathrm{A}, \mathrm{B}\}$. The prevention of the collapse due to the $\operatorname{ReplacE}\left(\mathcal{M}_{1}, \mathrm{~A}, \mathrm{~B}\right)$ operation can be seen when comparing the expected model count 7 with the actual model count after the REPLACE operation $M C\left(\mathcal{M}_{R}\right)=7$. This collapse prevention is accomplished due to the use of weighted transitions in the WFSA where the REPLACE algorithm changes the one $\mathrm{A}$ transition in $\mathcal{W}_{1}^{1}$ and the two $\mathrm{A}$ transitions in $\mathcal{W}_{1}^{2}$ to one and two $\mathrm{B}$ transitions respectively. Since these B transitions are duplicates of already exiting 
transitions, the minimize automata operation merges these duplicate transitions. However, since WFSAs have weighted transitions, the merging of the duplicates in the WFSA minimization algorithm requires the summation of the duplicate transition weights to produce the weight $2 \mathrm{~B}$ transitions seen in WFSA $\mathcal{W}_{R}^{1}$ and $\mathcal{W}_{R}^{2}$. Thus the $\mathrm{A}$ and $\mathrm{B}$ string values accepted by $\mathcal{W}_{1}^{1}$ are represented by the two $\mathrm{B}$ string values accepted by $\mathcal{W}_{R}^{1}$. Similarly, the AA, AB, BA, and BB string values accepted by $\mathcal{W}_{1}^{2}$ are represented by the four $\mathrm{BB}$ values accepted by $\mathcal{W}_{R}^{2}$. It is through the use of these weighted transitions that weighted automata model prevent collapses due to substitutive string operations. 


\section{CHAPTER 4}

\section{STRING CONSTRAINT SOLVER FRAMEWORK}

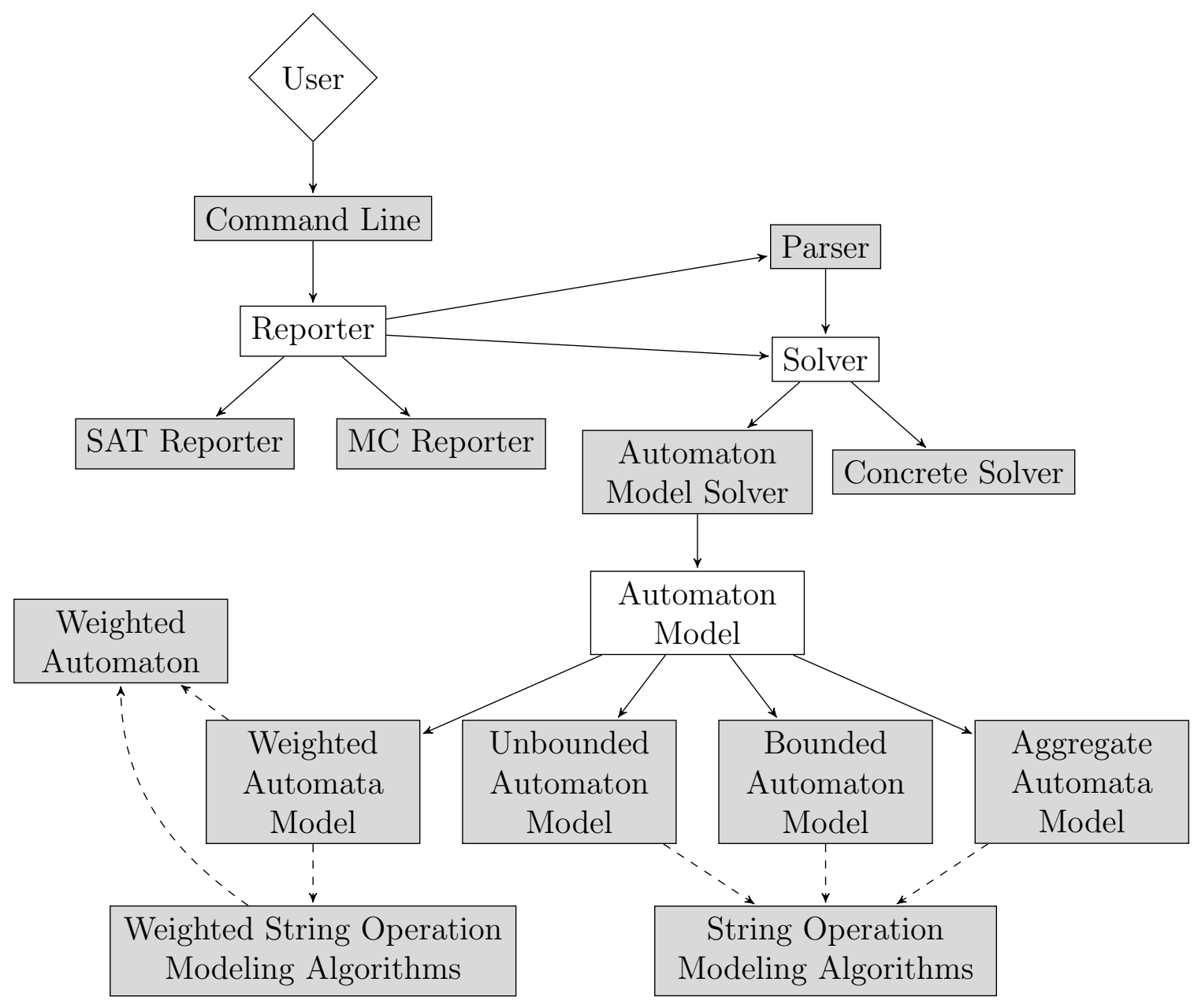

Figure 4.1: The String Constraint Solver Framework (SCSF)

The String Constraint Solver Framework (SCSF) is a Java software program which is used to solve a specified string constraint directed graph and determine either 
the satisfiability of the constraint or its model count depending on which option is specified. SCSF was adapted from the Processor component of the String Solver Analysis Framework detailed by Kausler [21]. This chapter will cover the different components used in the construction of this software analysis tool. Section 4.1 reports the third party software libraries upon which SCSF depends. Section 4.2 details the user interface to the SCSF tool. Section 4.3 explores the string constraint graphs (SCG) used as input to the SCSF tool. Section 4.4 describes the different components and their role in the software architecture of the SCSF tool. Section 4.5 outlines the test suite which ensures the correct functioning of the different components of the SCSF tool. Finally, Section 4.6 covers the various utility scripts used to orchestrate and supplement the SCSF tool in the analysis produced by this work.

\subsection{Third Party Dependencies}

\begin{tabular}{|c|c|c|}
\hline Library & Copyright Holder & Version \\
\hline Apache Maven & The Apache Software Foundation & \\
\hline Apache Commons CLI & The Apache Software Foundation & 1.3 .1 \\
\hline Apache log4j & The Apache Software Foundation & 1.2 .17 \\
\hline Jackson Project & FasterXML, LLC & 2.7 .2 \\
\hline jgrapht & Barak Naveh and Contributors & 0.9 .1 \\
\hline dk.brics.automaton & Anders Møller & $1.11-8$ \\
\hline Java String Analyzer & Anders Møller, Aske Simon, and Asger Feldthaus & $2.1-1$ \\
\hline JUnit & JUnit & 4.12 \\
\hline mockito & Mockito contributors & 1.10 .19 \\
\hline hamcrest & www.hamcrest.org & 1.3 \\
\hline NumPy & NumPy Developers & 1.9 .2 \\
\hline SciPy & Enthought, Inc. & 0.16 .0 \\
\hline
\end{tabular}

Table 4.1: SCSF Third Party Dependencies

In the creation of the SCSF tool and its associated utility scripts, we utilized 
many third party software libraries to speed the development process of the tool and to leverage the domain expertise embedded in these libraries. Table 4.1 lists each of these third party dependencies with the copyright holder associated with the library and the version used in the SCSF tool. The uses of each of these third party dependencies in the SCSF tool and associated scripts is listed below:

- The Apache Maven build automation tool allows easy tracking and updating of the third party dependencies in the SCSF tool.

- The Apache Commons CLI is used easily create a robust and standardized command line interface to the SCSF tool.

- The Apache log4j library is used to provide logging messages for the SCSF tool.

- The Jackson Project JSON library and parser which is used to parse the input SCG files which are expected to be in the JSON file format.

- The jgrapht graph theory library is used to construct the SCGs as Java objects in memory for processing in the SCSF tool.

- dk.brics.automaton is an automaton library used to create FSA for the unbounded, bounded, and aggrgate automata model implementations in the SCSF tool.

- Java String Analyzer is a string analysis tool which it includes a library of string operation and predicate simulation algorithms for dk.brics.automaton FSAs.

- JUnit is a unit testing framework used to create the unit test suite that accompanies the SCSF tool.

- mockito is a mocking framework used to create the test doubles for the unit tests in the test suite that accompanies the SCSF tool.

- hamcrest is library which is used to create easy to understand matching assertion critera for the unit tests in the test suite that accompanies the SCSF tool. 
- NumPy is a python library which enables the use efficient use of large arrays and matrices, it is used in the python utility scripts to gather and analyze the results of the evaluation described in Chapter 5.

- SciPy is a python library used for scientific and technical computing, it is used in the python utility scripts to perform statistical tests for the result analysis in the evaluation described in Chapter 5.

\subsection{Interface}

$\begin{array}{clcl}\text { SCG File Path } & \langle\text { filepath }\rangle & \text { Initial Bounding Length: } & \langle\text { integer }\rangle \geq 0 \\ \text { Reporter Type: } & \text { Satisfiability } & \text { Automata Model Type: } & \text { Unbounded } \\ & \text { Model Count } & \text { Bounded } \\ \text { Solver Type: } & \text { Concrete } & \text { Aggregate } \\ & \text { Automaton Model } & \text { Weighted }\end{array}$

Figure 4.2: SCSF Command Line Parameters

The command line processor component of SCSF uses Apache Commons CLI library to parse command line options and perform the appropriate configuration actions, e.g., converting the SCG file into an in-memory directed graph data structure. SCSF has one required command line parameter, a file path to a SCG file. The SCG file is expected to be a .json file adhering to a particular JSON schema and will be discussed in more detail in Section 4.3.1. SCSF also accepts five additional optional parameters which can be used to change the behaviour of the SCSF tool when solving the string constraints in the specified SCG. Without these optional parameters specified, default values are supplied instead. These five total command line parameters are listed and enumerated in Figure 4.2. The initial bounding length 
parameter can be used to refine the solver behaviour. The initial bounding length parameter specifies a maximum length for unknown string types when represented by symbolic string models with a default bounding length of 10 . The reporter type parameter determines whether the SCSF will solve the constraints for either satisfiability which is the default option or for probability, i.e., SE vs PSE. The solver type parameter specifies which string constraint solver to utilize, the options being either the default option of the automata model solver or the concrete solver oracle. Finally, if the automaton model solver type is chosen, an automta model type parameter can be used to choose an automata model with the default model as the unbounded automata model.

\subsection{Inputs}

The only input to the SCSF tool is a string constraint graph (SCG) for which the chosen string analysis will be performed. As mentioned in Section 4.2, SCSF requires the SCG file to be provided as a .json file. This file must also adhere to a specific JSON schema so that the edge and node data of the graph can be accurately converted into an in-memory graph data structure. This graph schema has emerged from the conversion of existing serialized graph files from the work of Kausler [21] into JSON files and thus reflects a string constraint SCG obtained via Dynamic Symbolic Execution (DSE) [11,33], i.e. each vertex in the SCG was recorded during the symbolic execution of a concrete software program. Section 4.3.1 describes this schema by detailing the required structure of an SCG. Section 4.3.2 then describes the creation of multiple synthetic SCGs which are used as the primary dataset for this analysis of automata-based symbolic string models. 


\subsubsection{Constraint Graph Structure}

\begin{tabular}{|c|c|c|c|}
\hline \multirow[t]{2}{*}{ Alphabet: } & Size & $\langle$ integer $\rangle \geq 0$ & \\
\hline & Alphabet Declaration & $\langle$ Alphabet Declaration $\rangle$ & \\
\hline \multirow[t]{11}{*}{ Verticies: } & Incoming Edges & & \\
\hline & & Source Vertex Id & $\langle$ integer $\rangle \geq 0$ \\
\hline & & Source Type & $\{\mathrm{t}, \mathrm{s} 1, \mathrm{~s} 2, \mathrm{~s} 3$ \\
\hline & Source Constraints & & \\
\hline & & Vertex Id & $\langle$ integer $\rangle \geq 0$ \\
\hline & Time Stamp & $\langle$ integer $\rangle \geq 0$ & \\
\hline & Value & $\langle$ StringConstraint $\rangle$ & \\
\hline & Number & $\langle$ integer $\rangle \geq 0$ & \\
\hline & Actual Value & $\langle$ string $\rangle$ & \\
\hline & Type & $\langle$ integer $\rangle \geq 0$ & \\
\hline & Id & $\langle$ integer $\rangle \geq 0$ & \\
\hline
\end{tabular}

Figure 4.3: SCG File Components

The schema for a graph file specifies two main components: an optional alphabet specification and an array of graph vertices. Both of these components are encapsulated in an outer JSON object. The characteristics of both the alphabet and vertices components are listed in Figure 4.3. The alphabet specification is a JSON object named alphabet and contains a maximum length and a symbol set specification. The maximum length has the key size and an integer value, e.g. "size":4. The symbol set specification in the JSON file has the key declaration and a string value which expresses the characters in the alphabet in the same way as bracket expression in a regular expression, e.g. "declaration" : A-Ca-C" which specifies only the characters A, B, C, a, b, and c are in the alphabet. If an alphabet specification is not included in the graph file, one is generated from the known string values which appear within the 
constraint graph. The vertices in the graph file are JSON objects which are contained in an array. Each vertex object contains the following items: an incoming edges array, a source constraints array, a time stamp, a value, a number, an actual value, a type, and an id. The array of incoming edges contains objects with two data items: a source vertex id corresponding the the id of another vertex object and a source time string value indicating that the current vertex object is either the primary $(t)$ or non-primary (s1-s6) parameter of a operation or predicate. The source constraints array contains the ids of other vertex objects visited before the current vertex was recorded. The time stamp value is the time at which the current vertex was recorded during the SCG creation process. The value is a string representation of the string constraint. The number is an integer indicating the number of previous visited constraints along the execution path before the current vertex. The actual value is the record of the actual string value the string variable corresponding to the current vertex recorded during the dynamic symbolic execution. The type is a integer value from 0 to 11 indicating a category of string constraint applying to the vertex, this value is not used in the SCSF tool and is a legacy of the previous work [21] upon which the SCSF tool is built. The id value is a positive integer which uniquely identifies the vertex object within the array of vertices. Both the alphabet specification and the vertices array are the only components of a JSON graph file expected as the input to the SCSF tool. 


\title{
4.3.2 Synthetic Constraint Graphs
}

\author{
Alphabet: 〈Alphabet Declaration〉 Input String(s): Specified Concrete String(s) \\ Maximum Consecutive \\ Empty String $(\varepsilon)$ \\ String Operations: $\langle$ integer $\rangle>0 \quad$ Unknown String
}

Figure 4.4: Synthetic SCG Generation Script Options

The synthetic SCG dataset is generated from a Python script, which produces one or more SCGs depending on the specified options. The script accepts a number of options, which configure the resulting SCGs. A user can configure which string operations and predicates are used to produce the SCGs. Other important script options include input string options, an alphabet specification, and the initial string length $k$. The input string options allows a user to specify one or more concrete string values as the initial strings values appearing at the root node in the SCGs. Additionally, two unique string values can be chosen as input strings: the empty string and an unknown string value (represents any string in the the specified alphabet up to $k$ ). The alphabet specification is used to generate both appropriate arguments for string operations which appear in the string constraints as well as generating random strings for the actual value field of the SCGs. The initial string length is used in conjunction with the unknown string value to limit the possible length of the random string generated for the actual value string.

The script is used to produce multiple string constraint graphs containing a thorough combination of possible string operations and predicates. The script begins by producing root nodes for the constraint graph corresponding to each of the specified input string options. Next, each configured string operation is used to produce an array of string operation nodes where each operation produces a thorough set of op- 
eration nodes for each possible argument configuration. For example, the substring operation will produce an operation for each of the following argument configurations when the initial string length is 2 : $\{(0,0),(0,1),(0,2),(1,1),(1,2),(2,2)\}$. Each of these string operation nodes are collected into a set for later use. String predicates undergo a similar process and are collected into a separate set. The set of predicate nodes are then used to produce an $n$-fold Cartesian product up to a depth $(n)$ specified as a script option. The resulting Cartesian product set contains all possible combinations of string operations in all possible operation orders. This set is then combined with the set of string predicates, producing a set where all possible string operation configurations are constrained by all possible string predicates. In this way, the script attempts to anticipate all possible string constraint graphs which can occur in a software program for the specified alphabet, initial strings, and operation depth.

\subsection{Components}

The three primary components of the SCSF tool are the reporter which is detailed in Section 4.4.1, the parser which is discussed in Section 4.4.2, and the solver which is explored in Section 4.4.3. Additional major components of the SCSF tool include the automata models which are detailed in Section 4.4.4, the weighted-transition finite state automaton implementation explored in Section 4.4.5, and the symbolic string model algorithms discussed in Section 4.4.6.

\subsubsection{Reporters}

The reporter component of SCSF is responsible for gathering the result of solving each branch PC in the SCG and reporting these results to standard output. Due to 
this central role, the reporter becomes the primary orchestrating component. The reporter retrieves each node from the SCG and must determine if the node represents a root node (a new string variable in the SCG) an operation node (a string operation) or a constraint node (a string predicate). For each of these node types, the node is then passed to the parser using the appropriate method for the node type. If the node type was a constraint node, the reporter gathers the results from the parser's semantic action (solving the true and false branch $P C$ s). Once all nodes in the SCG have been parsed and all semantic actions have been performed, the reporter reports the gathered results to standard output. The abstract class Reporter is implemented in the SCSF tool by two different reporters: SATReporter for satisfiability analyses and MCReporter for quantitative analyses.

\section{Satisfiability Reporter}

The SATReporter is an implementation of the Reporter abstract class in the SCSF tool and is responsible for coordinating and reporting the results of a SE analysis. This reporter requires a jgrapht directed graph of string constraint vertices, a Parser implementation, and an implementation of the ExtendedSolver abstract solver (satisfiability solver). The SATReporter outputs the following information for each predicate graph vertex:

- The integer id of the vertex.

- The "actual value" string value for the predicate vertex.

- If the predicate is a singleton branching point, i.e. all involved string variables only represent single concrete string values.

- The satisfiability of the true predicate branch.

- The satisfiability of the false predicate branch. 
- Whether the predicate branches are disjoint, i.e. branches are disjoint if there is no overlap in their solution sets: $\mathcal{S}_{t} \cap \mathcal{S}_{f}=\varnothing$.

- A string representation of the previous string initializations, operations, and predicates.

\section{Model Count Reporter}

The MCReporter is an implementation of the Reporter abstract class in the SCSF tool and is responsible for coordinating and reporting on the results of a PSE analysis. This reporter requires a jgrapht directed graph of string constraint vertices, a Parser implementation, and an implementation of the ModelCountSolver solver interface. The MCReporter outputs the following information for each predicate graph vertex:

- The integer id of the vertex.

- The "actual value" string value for the predicate vertex.

- If the predicate is a singleton branching point.

- The satisfiability of the true predicate branch.

- The satisfiability of the false predicate branch.

- Whether the predicate branches are disjoint

- The accumulated time of previous variable initializations, operations, and predicates.

- The id of the previous initialization, operation, or predicate vertex for the primary string argument in the predicate.

- The model count corresponding to the symbolic string model of the incoming primary string variable in the predicate.

- The time to perform the previous variable intialization, operation, or predicate for the primary string variable in the predicate. 
- The model count of the true predicate branch.

- The time required to compute the model count for the true predicate branch.

- The time required to simulate the predicate for the true predicate branch.

- The model count of the false predicate branch.

- The time required to compute the model count for the false predicate branch.

- The time required to simulate the predicate for the false predicate branch.

- The amount of overlap between the true and false predicate branches, i.e. model count of the disjoint symbolic string model.

- A string representation of the previous string initializations, operations, and predicates.

\subsubsection{Parser}

The parser component of SCSF is responsible for determining the appropriate semantic action for each node in the SCG. The parser accomplishes this in three different ways, one way for each of the different node types: root, operation, and constraint. For root nodes, the parser determines the string type of the new string value so that the appropriate symbolic string model can be created by the solver. For operation nodes, the parser determines the string operation so that the appropriate operation simulating algorithm can be chosen by the solver. For constraint nodes, the parser determines the string predicate so that the appropriate predicate simulating algorithm can be chosen by the solver. Through interpreting these three different node types in the SCG, the graph structure is converted into a series of action to be performed by the solver. 


\subsubsection{Solvers}

The solver component of SCSF is responsible for managing the symbolic string values through a variety of actions which correspond to the parser's semantic actions. These actions include creating new symbolic string models, simulating string operations for symbolic string models, determining satisfiability of a symbolic string model, and determining the model count of a symbolic string model. This solver component implements the bridge design pattern so that the actions can be applied to a variety of possible symbolic string models and allowing the symbolic string model implementation to vary independent of the solver [15]. Two different solver implementations are available: a concrete solver and an automaton model solver.

\section{Concrete Solver}

The concrete solver ConcreteSolver provides the string constraint oracle and implements the ModelCountSolver interface and extends the ExtendedSolver abstract class allowing ConcreteSolver to serve as the solver component for either type of reporter in the SCSF tool. This concrete solver can provide an oracle for either SAT or MC solvers due to two distinct differences from other abstract solver implementations: it models symbolic strings using arrays of concrete string values and it invokes the actual string operations and predicates rather than simulating such operations using algorithms. The ConcreteSolver models symbolic strings using an array of all possible concrete string values. These arrays of string values are created according to the different string types described in Section 2.4.2 where the empty string type is an empty array, the empty string string type is a 1 element array where the only element is the empty string "", and the literal string type is a 1 element 
array where the only element is the string literal. The complex unknown string type is only created as the result of string operations and predicates and so is not needed. The simple unknown string type is created by creating an array filled with only one of each and every possible string value for the specified alphabet and bounding length. This requires significantly more memory than string constraint solvers which employ symbolic models, but guarantees accuracy since each possible string value is accounted for in the array. The solver performs string operations on each concrete string which precisely simulates the results of string operations on the possible concrete string values. However, this does require a significant performance cost due to performing operations for each possible combination of strings and operation arguments. Both the performance and memory cost associated with the concrete solver makes it an impractical choice for real world analysis, but sufficient to provide the satisfiability and model count oracle for our analysis of automata-based symbolic string models.

\section{Automaton Model Solver}

The automata model solver AutomtonModelSolver provides the string constraint oracle and implements the ModelCountSolver interface and extends the ExtendedSolver abstract class allowing ConcreteSolver to serve as the solver component for either type of reporter in the SCSF tool. The AutomatonModelSolver requires an initial bounding length and an Alphabet object which are used when creating initial symbolic string models. This solver also requires an implementation of the abstract AutomatonModelManager which is an abstract factory for producing AutomatonModel

objects which are further detailed in Section 4.4.4. The AutomtonModelSolver is responsible for coordinating the semantic actions assigned by the reporter, this means that the solver coordinates the initialization of a new symbolic string model, simulates 
a string operation, or simulates either the true or false branch of a string predicate. The initialization of new symbolic string models is accomplished by requesting the appropriate model from the AutomatonModelManager object. The simulation of string operations is accomplished by requesting the appropriate operation simulation from the AutomatonModel corresponding to the primary string argument of the operation. The simulation of either true or false branches of a predicate is accomplished by requesting the appropriate operation simulation from the AutomatonModel corresponding to the primary string argument of the predicate. This coordination role of a AutomatonModelSolver allows the implementations of both the AutomatonModel factory and the AutomatonModel itself to vary independent of the solver, allowing the addition of future automaton models.

\subsubsection{Automaton Models}

The automaton model sub-component of SCSF is responsible managing automata models. The responsibilities of these automata models include the creation of new automata models, model counting, and simulating string operations and predicates for automata models. The automata model sub-component consists of an abstract automata model interface as well as concrete implementations of this interface for the four automata models discussed in detail in Chapter 3. The abstraction of an automata model is another implementation of the bridge design pattern allowing the concrete automata model implementations to vary independent of the AutomatonModelSolver [15]. In this manner, the AutomatonModelSolver uses the abstract AutomatonModel as its symbolic string model rather than any specific automata model implementation allowing for greater flexibility when modifying or adding automata model implementations. This flexibility also allows future automata models to be implemented with lit- 
tle extra overhead. The four automata models implemented for the AutomatonModel interface are as follows: UnboundedAutomatonModel, BoundedAutomatonModel, AggregateAutomat: and WeightedAutomatonModel

\section{Unbounded Automata Model}

The UnboundedAutomatonModel is an implementation of the unbounded automata model described in Section 3.1. An UnboundedAutomatonModel contains an integer to track the maximum length, an Alphabet object to encapsulate the alphabet for the model, and a dk.brics.automaton. Automaton object as the FSA implementation. An UnboundedAutomatonModel object is responsible for determining its model count, simulating string operations where it is the primary argument, and simulating string predicates where it is the primary argument. An UnboundedAutomatonModel object does utilize algorithms external to the itself for model counting, operation simulation, and predicate simulation. These external algorithms operate upon dk. brics . automaton. Automator objects and therefore require only dk.brics.automaton. Automaton objects instead of AutomatonModel objects. Therefore, the aspects of model counting, operation simulation, or predicate simulation specific to unbounded automata models are handled within the UnboundedAutomatonModel object.

\section{Bounded Automata Model}

The BoundedAutomatonModel is an implementation of the bounded automata model described in Section 3.2. A BoundedAutomatonModel is comprised of an integer bounding length of the FSA, an Alphabet object to encapsulate the alphabet for the model, and a dk.brics.automaton. Automaton object as the FSA implementation. A BoundedAutomatonModel object is responsible for determining its model count, 
simulating string operations where it is the primary argument, and simulating string predicates where it is the primary argument. A BoundedAutomatonModel object also uses external algorithms for model counting, operation simulation, and predicate simulation. These external algorithms are the same algorithms used by the UnboundedAutomatonModel since both it and UnboundedAutomatonModel use the same dk. brics. automaton. Automaton objects for their FSAs. Similarly, the aspects of model counting, string operation simulation, or string predicate simulation specific to a bounded automata model are handled within the UnboundedAutomatonModel object.

\section{Aggregate Automata Model}

The AggregateAutomataModel is an implementation of the aggregate automata model described in Section 3.3. An AggregateAutomataModel object includes an integer to track the bounding length of the model, an Alphabet object to encapsulate the alphabet for the model, and an array of a dk.brics.automaton. Automaton objects as the FSA sequence implementation. An AggregateAutomataModel object is responsible for determining its model count, simulating string operations where it is the primary argument, and simulating string predicates where it is the primary argument. An AggregateAutomataModel object also uses external algorithms for model counting, operation simulation, and predicate simulation. These external algorithms are the same algorithms used by both the UnboundedAutomatonModel and the BoundedAutomatonModel since all three use the same dk. brics . automaton. Automaton objects for their FSAs. Similarly, the aspects of model counting, string operation simulation, or string predicate simulation specific to a aggregate automata model are handled within the AggregateAutomataModel object. 


\section{Weighted Automata Model}

The WeightedAutomatonModel is an implementation of the weighted automata model described in Section 3.4. A WeightedAutomatonModel contains an integer to track the bounding length of the model, an Alphabet object to encapsulate the alphabet of the model, and an array of WeightedAutomaton objects as the WFSA sequence implementation. A WeightedAutomatonModel object is responsible for determining its model count, simulating string operations where it is the primary argument, and simulating string predicates where it is the primary argument. A WeightedAutomatonModel also uses external algorithms for model counting, operation simulation, and predicate simulation. Unlike the other three automata model implementations, a WeightedAutomatonModel object uses external algorithms which operate upon WeightedAutomaton objects. The the aspects of model counting, string operation simulation, or string predicate simulation specific to a weighted automata model are handled within the WeightedAutomatonModel object.

\subsubsection{Weighted-Transition Finite State Automaton}

The WeightedAutomaton is an implementation of the weighted-transition finite state automaton defined in Section 3.4.1. A WeightedAutomaton object includes both an integer serving as the empty string counter $i_{\varepsilon}$ and a WeightedState object which servers as the initial state $q_{0}$ in the WFSA. This initial state object is the only state contained within WeightedAutomaton object, the other WeightedState objects acting as states in the WFSA are reached from this initial WeightedState. A WeightedState includes a boolean variable to mark the state as accepting and a set of WeightedTransition objects which are the outgoing transitions from the WFSA 
state. A WeightedTransition includes a minimum char, a maximum char, an integer transition weight, and a destination WeightedState. By using both minimum and maximum char values, a single WeightedTransition can represent multiple WFSA transitions for adjacent symbols. It is the combination of the WeightedAutomaton class, the WeightedState class, and the WeightedTransition class that the implementation of a WFSA is achieved in the SCSF tool. While this WFSA structure is useful in the SCSF tool, using WFSAs as part of an automata model requires working automata operation algorithms for some essential operations. The algorithms implemented for the SCSF tool include: Brzozowski's minimization [6], DETERminizAtion (subset construction), Intersection, Union, Subtraction, and Concatenation.

\subsubsection{Symbolic String Model Algorithms}

The collection of symbolic string constraint algorithms is best divided into three separate types of algorithms: model counting algorithms (Section 4.4.6), string and predicate simulation algorithms using FSAs, and string and predicate simulation algorithms using WFSAs.

\section{Model Counting Algorithms}

Because the model counting algorithms discussed in Sections 3.1.1, 3.2.1, 3.3.2, and 3.4.3 are specific to each automata model, the portions of each procedure specific to an automata model, i.e. Countunbounded, CountBounded, CountAggreGATE, and CountWeighted, are encapsulated within their respective AutomatonModel implementations. However, the algorithms specific only to either FSAs ore WFSAs, i.e. MCUnbounded, MCBounded, and MCWEighted, were implemented as al- 
gorithms separate from the AutomatonModel classes. The StringModelCounter class in the SCSF tool provides implementations for each of these FSA or WFSA specific algorithms. These model counting algorithms each utilize and return BigInteger object to allow for much larger integer values which can occur due to longer string lengths and larger alphabets.

\section{FSA Algorithms}

While the existing string and predicate operation simulation algorithms from the Java String Analyzer library [12] were sufficient for SE analyses, some operations and predicates required more precise algorithms to be useful in PSE analyses. The SCSF tool includes more precise versions of the following string operation simulation algorithms: Delete, Insert, Prefix, SetCharAt, Setlength, Substring, Suffix, and TRIM.

\section{WFSA Algorithms}

Since WeightedAutomaton objects did not have either the Java String Analyzer library to provide string operation and predicate simulation algorithms, such algorithms were created to operate on WeightedAutomaton objects. Some operations and predicates have both precise and imprecise simulation algorithms for WeightedAutomaton objects, specifically: Prefix, RePlace (for single symbol arguments), Substring, and SufFIX. Other operations and predicates with precise simulation algorithms for for WeightedAutomaton objects include: Delete, Insert, SetCharAt, SetLength, and TRIM. In addition to these precise algorithms, the following string operations and predicates have simulation algorithms for WeightedAutomaton objects included 
in the SCSF tool: RePlace (for string arguments), Reverse, ToLowerCASE, and TOUpperCase.

\subsection{Test Suite}

The SCSF test suite contains 6635 unit tests covering the concrete solver, the four automata models, the WFSA implementation, and the symbolic string algorithms created for the SCSF tool. The test suite does not cover the other components of the SCSF tool either because the component is a legacy component from the String Solver Analysis Framework [21] or because the component is primarily serving a straightforward coordinator which is not complex enough to benefit from unit testing coverage. The unit test in the SCSF test suite were created primarily as part of a test-driven-development [2] and follow a Given-When-Then class and method style [14] to aid comprehension of each unit test. To ensure the independence of the objects under test, the mockito library is used to provide test doubles for the interfaces and abstract classes upon which many components within SCSF tool depend. This test suite is able to provide easy to understand verification that the components are correct and perform as expected indecent of the evaluation detailed in Chapter 5.

\subsection{Utility Scripts}

The final portion of the SCSF tool is actually external to the tool. The utility scripts accompany the SCSF tool and consist of five primary Python script files and other supporting utility Python scripts which automate the evaluation described in Chapter 5. One of these scripts, run.py, orchestrates the other four automation scripts allowing the entire evaluation process to be invoked using a single script with 
appropriate command line arguments specified (documentation of the command line arguments is built into this and all other scripts). The four other primary scripts are: generate_graphs.py, run_solvers_on_graphs.py, gather_results.py, and analyze_results.py. The generate_graphs.py script automates the generation of the synthetic SCG dataset discussed in Section 4.3.2. The run_solvers_on_graphs .py script automates the execution of the SCSF tool on the specified set of graphs using the specified different reporters and solvers. The gather_results.py script automates the collection of the result data from the SCSF tool and generates a single result data file fore each automata model. Finally, the analyze_results.py script automates the statistical analysis of the result data and generates the appropriate LaTeX output data tables and gnuplot plots for this data. 


\section{CHAPTER 5}

\section{EVALUATION}

In order to evaluate the suitability of different automata models for modeling symbolic string constraints in PSE, each automata model is evaluated in a set of controlled experiments. These experiments consist of each automata model and a model counting oracle solving a series of synthetic string constraints. The result data from these experiments is used to measure the accuracy and performance of each automata model in PSE analyses. Additionally, the independent variables of the evaluation (detailed later in Section 5.2) are analyzed in isolated experiments to determine their effects on accuracy and performance for the automata models.

This chapter is organized as follows. Section 5.1 details the procedure used to conduct the evaluation. Section 5.2 explains the independent variables of the evaluation. After that, the next two sections of this chapter describe the measurements recorded in the evaluation experiments which are be used to determine the suitability of the automata-based constraint solvers: Section 5.3 describes the measurement of accuracy and Section 5.4 describes the measurement of performance. Section 5.5 reports the additional data analyses which compare accuracy and performance metrics. Finally, Section 5.6 concludes the chapter by detailing the evaluation environment. 


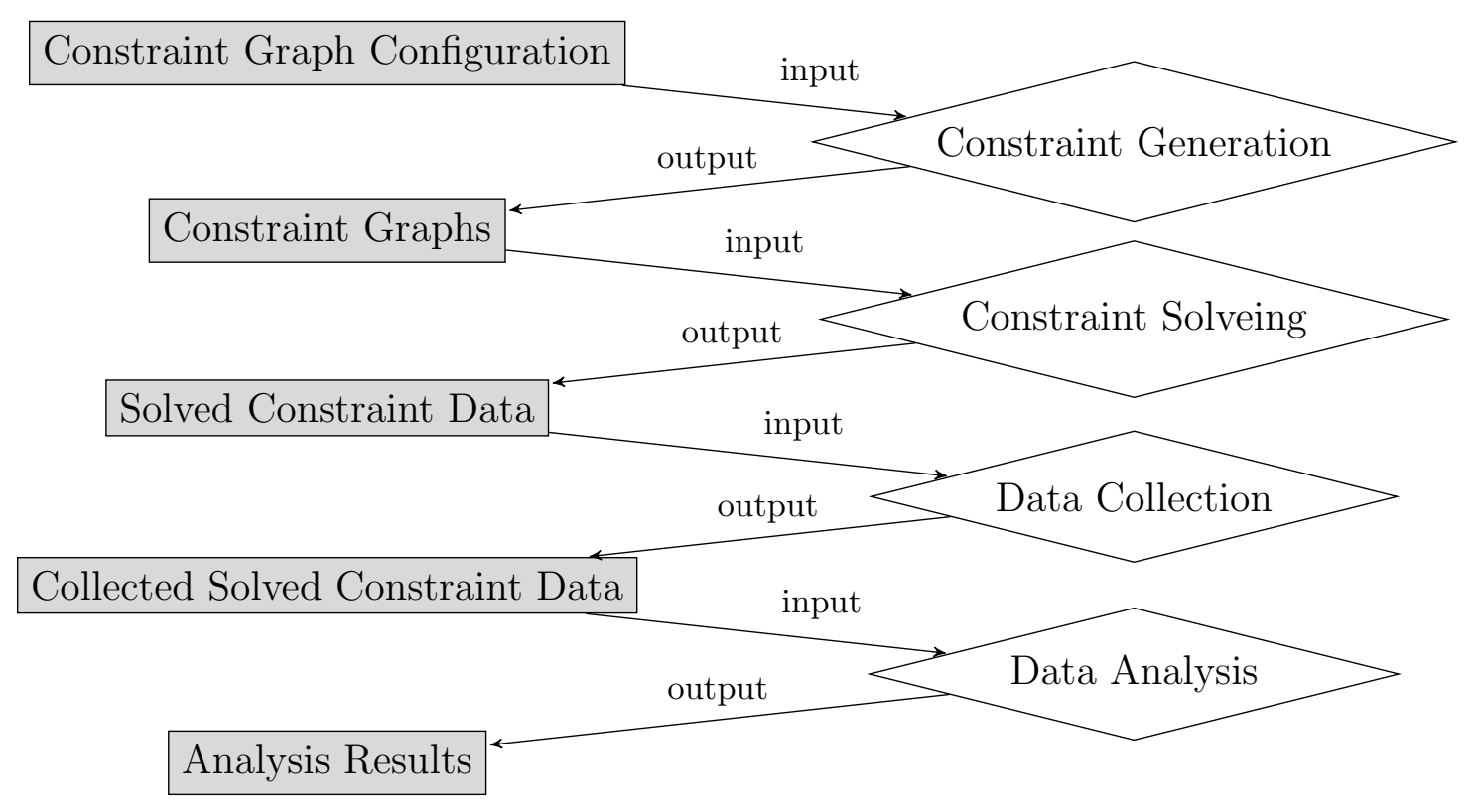

Figure 5.1: The Evaluation Procedure

\subsection{Evaluation Procedure}

Figure 5.1 is a diagram illustrating the evaluation procedure and the associated input and output data for each step in the procedure. The four diamonds on the right side of the diagram and consist of the Constraint Generation, Constraint Solving, Data Collection, and Data Analysis steps in the evaluation. The five grey rectangles on the left side of the diagram represent the different inputs and outputs for each step of the evaluation procedure where the output of one step is becomes the input of the next step in the evaluation, except for the the output of the Data Analysis step which are the results of the evaluation and are discussed in Chapter 6 .

\subsubsection{Constraint Generation}

The Constraint Generation step is responsible for creating the inputs to the evaluation's controlled experiments. These inputs are the Constraint Graphs and 
consist of multiple sets of synthetic string constraints that are generated as graphs in the format described in Section 4.3. A constraint graph is created from a collection of independent constraint subgraphs. These sub-constraints are in turn created from a combination of parameters as specified by the Constraint Graph Configuration. These parameters are the independent variables in the evaluation and are later explained in Section 5.2. The graph generation begins by reading each the Constraint Graph Configuration into memory. Next, nodes representing operations and predicates and edges representing the targets and arguments of the operations and predicates are created according to the configuration parameters. These nodes and edges form string constraint graph structures which correspond to the individually constraints to be analyzed in the PSE analysis. These constraints are then collected into the Constraint Graphs which consist of multiple JSON files. The Constraint Generation step is automated by a python script for repeatability and ease of use. Next, constraint solvers use these constraint graphs in the Constraint Solving step of the evaluation procedure.

\subsubsection{Constraint Solving}

The Constraint Solving step is responsible for performing the PSE analyses on each of the Constraint Graphs. Each PSE analysis of a graph is conducted for each of the four automa-based constraint solvers as well as the concrete solver oracle. These PSE analysis are performed by SCSF (Chapter 4). This process invokes SCSF for each combination of generated graph and solver. Each invocation of SCSF produces a tab-delimited text file as Solved Constraint Data. The data columns reported by SCSF are listed and explained in Table 5.1. The Constraint Sequence column values are of special note because of the additional data embedded in these values which are 


\begin{tabular}{|l|l|}
\hline Column & Explanation \\
\hline Constraint Id & $\begin{array}{l}\text { The integer which uniquely identifies the constraint within its parent } \\
\text { constraint graph. This id corresponds to the node id of branch predicate } \\
\text { of the constraint graph. }\end{array}$ \\
\hline Constraint Sequence & $\begin{array}{l}\text { A string value specifying the sequence of operations and predicates which } \\
\text { comprise the sub-constraint. This value contains additional information } \\
\text { about the operations and predicates in the sequence. }\end{array}$ \\
\hline Cumulative Time & $\begin{array}{l}\text { The total time required to perform all dependent string operations and } \\
\text { string predicates. }\end{array}$ \\
\hline Before MC & $\begin{array}{l}\text { The model count before the terminal predicate of its target symbolic } \\
\text { string. This model count represents the number of execution paths } \\
\text { reaching the predicate. }\end{array}$ \\
\hline false MC & The model count of the false branch of the constraint. \\
\hline false MC Time & $\begin{array}{l}\text { The time required to calculate the model count of the symbolic string } \\
\text { model representing the false branch of the constraint. }\end{array}$ \\
\hline false Predicate Time & $\begin{array}{l}\text { The time required to model the false branch predicate for the two } \\
\text { symbolic string arguments. }\end{array}$ \\
\hline true MC & The model count of the true branch of the constraint. \\
\hline true MC Time & $\begin{array}{l}\text { The time required to calculate the model count of the symbolic string } \\
\text { model representing the true branch of the constraint. }\end{array}$ \\
\hline true Predicate Time & $\begin{array}{l}\text { The time required to model the true branch predicate for the two symbolic } \\
\text { string arguments. }\end{array}$ \\
\hline
\end{tabular}

Table 5.1: Data Columns Reported For Solved Constraints

extracted later in the evaluation procedure. The Constraint Solving step is automated by another python script and produces a text file for each combination of generated graph and symbolic model.

\subsubsection{Data Collection}

The Data Collection step is responsible for gathering all of the individual result text files which constitute the Solved Constraint Data and partitions them into three data groups: model counting accuracy, constraint solving performance, and model counting performance. The collection step begins by reading the result text files into memory. Next, the data tables are grouped by the constraint graphs from which the result tables were produced. This grouping ensures that the Constraint Id values in 


\begin{tabular}{|l|l|}
\hline Column & Explanation \\
\hline File & $\begin{array}{l}\text { The name of the result file containing the data result. This corresponds to } \\
\text { the generated graph solved to produce the data result. }\end{array}$ \\
\hline String Type & $\begin{array}{l}\text { The type of the initial input string of the constraint as described in Section } \\
2.4 .2 .\end{array}$ \\
\hline Operation 1 & The first operation in the constraint. \\
\hline Operation 1 Arg & $\begin{array}{l}\text { The category of argument combination received by the first operation in } \\
\text { the constraint. }\end{array}$ \\
\hline Operation 2 & The second operation in the constraint. \\
\hline Operation 2 Arg & $\begin{array}{l}\text { The category of argument combination received by the second operation in } \\
\text { the constraint. }\end{array}$ \\
\hline Predicate & The branch predicate of the constraint. \\
\hline Predicate Arg & $\begin{array}{l}\text { The input string type of the second string argument for the branch predicate } \\
\text { of the constraint. }\end{array}$ \\
\hline
\end{tabular}

Table 5.2: New Data Columns Extracted from Constraint Sequence Values

each group can be used to identify the same sub-constraint solved using each of the five solvers. Next, an intermediate processing step parses each Constraint Sequence value for each data row and extracts the values for 7 new data columns which are added to the results tables. An additional File data column is also added to the result tables. These 8 new data columns are defined and explained in Table 5.2. Next, the result tables in each constraint graph group are partitioned into three subtables: a model count data table, a constraint solving performance data table, and an operation and predicate performance data table. In both the model count table and the constraint solving table, each data rows corresponds to a single sub-constraint solved during the PSE analysis. The separate model count data for each of the five solvers are appended to a single output data row under separate data columns. Similarly, the separate constraint solving performance results are appended for each of the solvers. The operation and predicate performance data is also appended to a row, but in this output data table, each row corresponds to a specific operation or predicate in each constraint instead of the constraint itself. Finally, the groups of these subtables 
are joined to form three result data tables as the Collected Solved Constraint Data. This Data Collection step is automated as another python script and produces three tab-delimited text files for processing in the Data Analysis step.

\subsubsection{Data Analysis}

The Data Analysis step is responsible for transforming the Collected Solved Constraint Data into information useful for determining the suitability of the four automatabased solvers in PSE analyses. This Data Analysis will also evaluate the effects of the independent variables (detailed later in Section 5.2) on the suitability of each solver. The Data Analysis begins by reading the three text files into memory. Next, informative measurements are taken for solver accuracy and performance. The details of these measurements are provided later in Section 5.3 for accuracy and Section 5.4.2 for performance. The Data Analysis is also automated by a python script to ensure repeatability and produces Analysis Results.

\subsection{Independent Variables}

The five independent variables of the evaluation listed along with their values in Table 5.3. These variables are introduced in the evaluation through representations in the synthetic Constraint Graphs. These independent variables correspond to five characteristics of string constraints discussed in Sections 2.4.2, 2.4.2, and 2.4.2. So that this evaluation is relevant for PSE analyses on actual software programs, the synthetic graphs are generated according to the format described in Section 4.3. The nodes and edges of the graphs are generated representing the independent variable values specified as the Constraint Graph Configuration parameters. The representation of 


\begin{tabular}{|c|c|c|}
\hline Variable & \multicolumn{2}{|l|}{ Values } \\
\hline String Alphabet & $\multicolumn{2}{|c|}{\mathrm{A}, \mathrm{B}},\{\mathrm{A}, \mathrm{B}, \mathrm{C}\},\{\mathrm{A}, \mathrm{B}, \mathrm{C}, \mathrm{D}\},\{\mathrm{A}, \mathrm{B}, \mathrm{C}, \mathrm{D}, \mathrm{E}\}$ \\
\hline Initial Maximum String Length & \multicolumn{2}{|l|}{$1,2,3,4$} \\
\hline Initial Input String Type & \multicolumn{2}{|l|}{ Literal, Simple, Complex } \\
\hline Constraint Operations & $\left(\begin{array}{c}\text { reverse }() \\
\text { concat }(\text { Literal }) \\
\text { concat }(\text { Simple }) \\
\text { concat } \text { (Complex }) \\
\text { delete }(\text { Same }) \\
\text { delete }(\text { Different }) \\
\text { replace(Same }) \\
\text { replace }(\text { Different })\end{array}\right)$ & $\times\left(\begin{array}{c}\text { none } \\
\text { reverse }() \\
\text { concat }(\text { Literal }) \\
\text { concat }(\text { Simple }) \\
\text { concat }(\text { Complex }) \\
\text { delete }(\text { Same }) \\
\text { delete }(\text { Different }) \\
\text { replace }(\text { Same }) \\
\text { replace }(\text { Different })\end{array}\right.$ \\
\hline Constraint Predicate & \multicolumn{2}{|c|}{$\begin{array}{l}\text { contains }(\text { Literal }) \text {, contains }(\text { Simple }) \text {, contains }(\text { Complex }) \text {, } \\
\text { equals }(\text { Literal }) \text {, equals }(\text { Simple }) \text {, equals }(\text { Complex })\end{array}$} \\
\hline
\end{tabular}

Table 5.3: The Independent Variables in the Evaluation

these independent variables in the graph nodes and edges is described in this section as follows: the string alphabet in Section 5.2.1, the initial maximum string length in Section 5.2.2, the initial input string type in Section 5.2.3, the string operations in Section 5.2.4, and finally, the string predicates in Section 5.2.5. Additionally, Section 5.2.6 describes the distribution of the independent variables in the synthetic graphs.

\subsubsection{String Alphabet}

The string alphabet for a constraint is the set of symbols in the language of the strings in the constraint. Section 2.4.2 discusses the properties of the string alphabet which characterizes a string constraint. The four string alphabet values which appear in this evaluation are listed in Table 5.3. In this evaluation, the string alphabet is treated as an interval quantitative variable for the size of the alphabet value. This is because the the algorithms which are used in the Constraint Solving step are limited 
by the size of the alphabet, not the actual symbols in the alphabet.

The alphabet variable is represented in constraint generation by restricting the concrete strings in constraints to the language of strings over the string operations. This variable is also represented in the Constraint Solving step by restricting the language of string values represented by the symbolic string models of the solvers.

\subsubsection{Initial String Length}

The initial maximum string length of an input strings is the maximum possible length for the string values represented by the primary input string in the constraint. For example, a string input variable is assigned from an external source in a program, the corresponding symbolic string value for this variable will represent all possible string values with a length between 0 and the initial maximum length. Section 2.4.2 describes the initial maximum string length as a property of the string length characteristic in a string constraint. The four initial maximum string length values which are used in this evaluation are listed in Table 5.3. In this evaluation, the initial maximum string length is an interval quantitative variable.

The length variable is represented in the synthetic graphs by ensuring the concrete strings in the graph are not longer than the maximum length. This variable is also used in the Constraint Solving step by restricting the input string values represented by the symbolic string models of the solvers to the initial maximum length.

\subsubsection{Input String Type}

The input string type of a constraint defines the distribution of the string values in the set represented by the symbolic string value. Section 2.4.2 explains the three types of input strings in detail. Table 5.3 lists these three types which are used in this 
evaluation. Table 5.3 shows these three values used specifically for the initial input string in a constraint. The initial input string type in a constraint is the type for the string variable which is the primary input string variable in the constraint. For example, in the constraint contains $\left(\operatorname{concat}\left(s_{1}, s_{2}\right), s_{3}\right)$, the string variable $s_{1}$ is this primary input string variable. In this evaluation, the input string type is a categorical variable because of its three unordered categories.

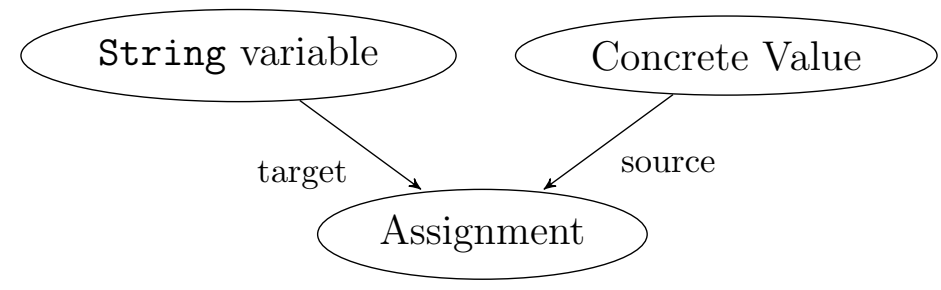

Figure 5.2: Literal Input String Type Graph Representation

Literal The created graph structure representing an Literal input string type is shown in Figure 5.2. There are three nodes in the structure: one for a string variable, one for an assignment operation, and one for the concrete string value. Two edges connect the string variable and concrete value nodes to the assignment operation node as target and source edges respectively. This structure represents the assignment of the concrete value to the string variable in a program.

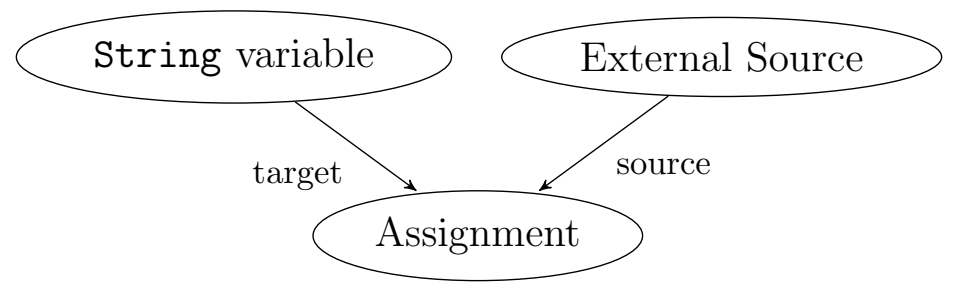

Figure 5.3: Simple Input String Type Graph Representation 
Simple The created graph structure representing an Simple input string type is shown in Figure 5.3. There are three nodes in the structure: one for the string variable, one for an assignment operation, and one for the call to an external process. An external process is any external source of a string value such as standard input, another program, etc.. The target and source edges connect the string variable and external source nodes respectively to the to the assignment operation. This structure represents an assignment of an unknown string value to the input string variable in a program.

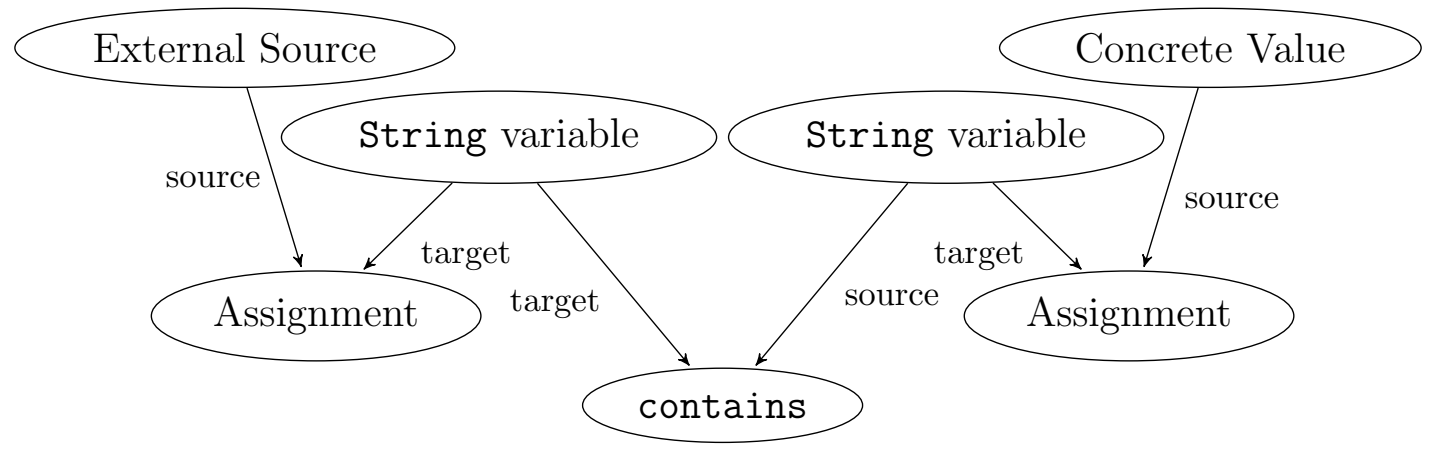

Figure 5.4: Complex Input String Type Graph Representation

Complex The created graph structure representing an Complex input string type is shown in Figure 5.4. The Complex string is constructed using an Simple string, a Literal string, and a contains operation node. The string variable node from the Simple structure is connected with an edge to the contains operation node as the primary string argument (target). The string variable of the Literal structure is connected with an edge to the contains operation as the second string argument (source). This structure for the Complex input string type results in the contains operation applied to the input string variable from the Simple string structure and therefore creating an complex distribution of string values represented for that string 
variable. As the creation process indicates, the Complex input string type is not strictly an initial string input type since it requires both a Literal and an Simple string type to construct. However, in this evaluation, the entire Complex structure is considered as representing the single Complex initial input string type.

\subsubsection{Operations}

The string operations in a constraint are the sequence of either one or two operations in the constraint. Section 2.4.2 explains how these operations characterize a constraint and describes the four string operation types included in the generated synthetic graphs: Injective, Additive, Subtractive, and Substitutive. Since each string operation in an operation category has similar effects on a symbolic string value, then only one operation from each category is represented in the constraints for this evaluation. These chosen operation are reverse for Injective operations, concat for Additive operations, delete for Subtractive operations, and replace with char arguments for Substitutive operations. Additionally, The concat, delete, and replace operations each takes a sequence of arguments. For each operation, the argument sequences can be categorized into distinct argument configurations based on the similar effects on a symbolic string value. The concat operation has three configurations for its single string argument: Literal, Simple, and Complex. The delete operation has two configurations for its two integer arguments: Same and Different. The replace operation has two configurations for its two character arguments: Same and Different. Table 5.3 shows values of the string operations independent variable in this evaluation where the variable values are the sequence of either one or two operations produced from the cross product shown in the table. The string operations variable is a nominal categorical variable due to the operation 
sequences being unordered category values.

One of the goals of this evaluation is to determine the effects of the different string operation types on the automata-based solvers. Unfortunately, the string operations variable values are operation sequences and can not be used directly to isolate the effects of specific operation types. Instead, different PSE constraint graph sets are used to determine these effects: graphs only containing one operation or operation argument configuration for all constraints and graphs including one operation or operation configuration for all constraints. These two series of experiments allows the effects of the string operation type to be isolated. While the string operation variable is used differently in these two series of experiments, it is still a nominal categorical variable because of the unordered category values.

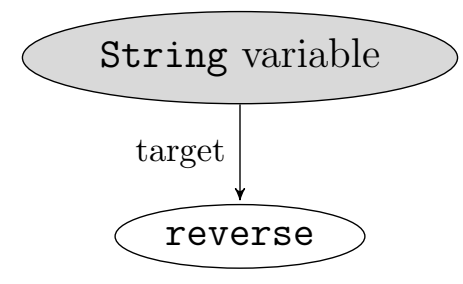

Figure 5.5: reverse Operation Graph Representation

reverse The created graph structure representing the reverse operation is shown in Figure 5.5. There are only two nodes in the structure: one for the target string variable and one for the reverse operation. There is a single target edge from the string variable to the reverse operation. This structure represents a reverse operation in the synthetic constraint graphs.

concat The created graph structure representing the concat operation is shown in Figure 5.6. There are three nodes in this structure: one for the target string variable, 


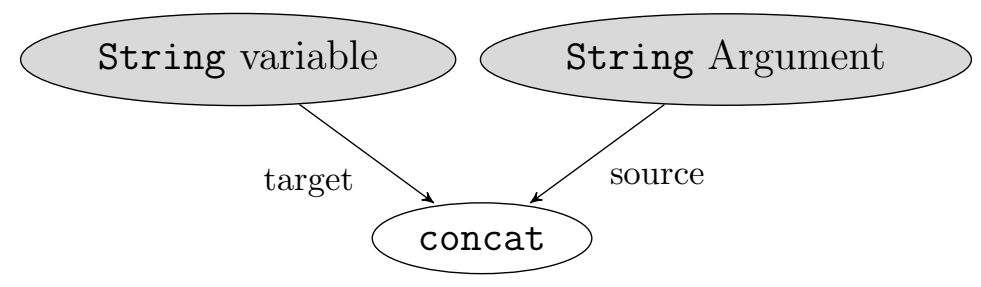

Figure 5.6: concat Operation Graph Representation

one for the argument string variable, and one for the concat operation. There are two edges in the structure: a target edge connecting the target string variable to the operation and an argument edge (source) connecting the string argument to the operation. The string argument node in this structure represent one of the three input string structures shown in Section 5.2.3: Literal, Simple, or Complex. This is the structure representing a concat operation in the synthetic graphs.

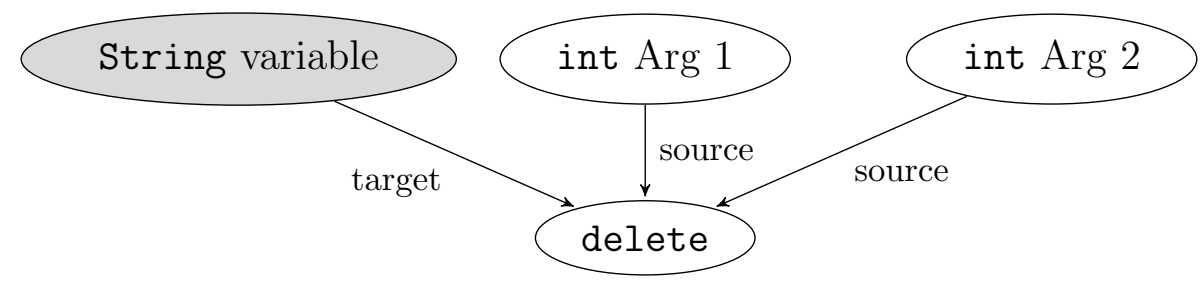

Figure 5.7: delete Operation Graph Representation

delete The created graph structure representing the delete operation is shown in Figure 5.7. There are four nodes in this structure: one for the target string variable, one for the first int argument (the start index), one for the second int argument (the end index), and one for the delete operation. The target edge connects the string variable to the operation node. Two source edges connect the two argument nodes to the operation node. These source edges are labeled s1 and s2 in the synthetic graph files. 


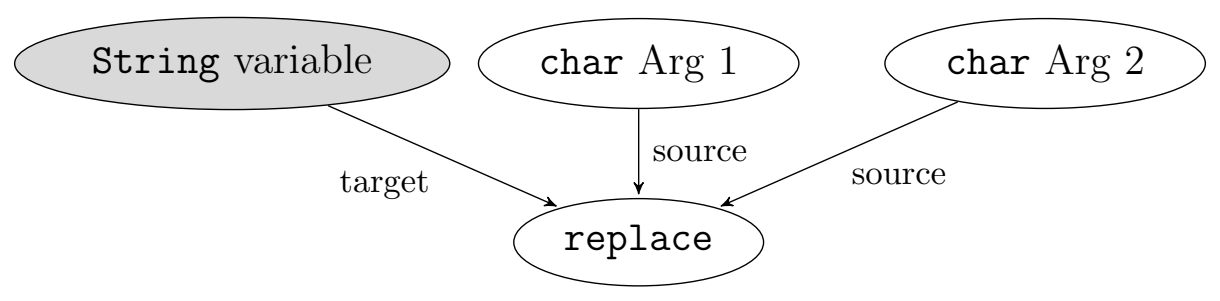

Figure 5.8: replace Operation Graph Representation

replace The created graph structure representing the replace operation is shown in Figure 5.8. This structure contains four nodes: one for the target string variable, one for the first char argument, one for the second char argument, and one for the replace operation. There is a target edge connecting the string variable and the operation. There are two source edges connecting the two argument nodes and the operation node. These source nodes are labeled s1 and s2 in the generated graph files.

\subsubsection{Predicates}

The predicates in a constraint are the terminal predicate as well as any other predicate in the constraint. In this evaluation, only the terminal predicates will be considered predicates since all other predicates in the Constraint Graphs are the contains predicates needed to create Complex input strings. Section 2.4.2 explains how predicates can characterize constraints and describes the two types of predicates which are used in this evaluation: Partial Match and Full Match predicates. One predicate is chosen from both categories to be included in the Constraint Graphs. The contains predicate represents the Partial Match category and the equals predicate represents Full Match category. Both of these predicates have a target a string and takes one string argument. The string argument in this evaluation is always one of three input string types: Literal, Simple, and Complex. Table 5.3 shows these six 
predicate configurations as the predicates variable values. The predicates variable is a nominal categorical variable in this evaluation due to the predicate configurations being unordered category values.

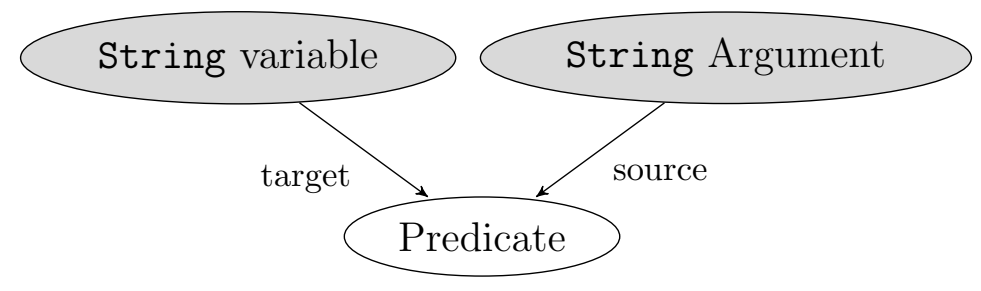

Figure 5.9: Predicate Graph Representation

Figure 5.9 shows the created graph structure for both the contains and equals predicates. This structure contains three nodes: one for the target string variable, one for the argument string variable, and one for the predicate. An edge connects the target string variable to the predicate while a source edge connects the argument string variable to the predicate. to the operation and an argument edge (source) connecting the string argument to the operation. The string argument node in this structure represent one of the three input string structures shown in Section 5.2.3. This structure is created to represent both the contains and the equals predicates in the synthetic graphs.

\subsubsection{Distribution of Independent Variables in Graphs}

Four of the five independent variables in this evaluation are evenly distributed through the Constraint Graphs so that the results of the experiments are not skewed by the effects of any particular variable value. These four variables are the string alphabet, the initial maximum string length, the initial input string type, and the predicates. For each string alphabet and initial maximum string length, a constraint graph file is generated where all constraints in the graph file have one of three initial 
input string variables with the corresponding alphabet and maximum string length. The three initial variables correspond to the three initial input string type values. Each of these graphs a all combinations of string operation sequences. Each of these sequences is duplicated twelve times so that each string predicate configuration is applied to all operation sequences.

\begin{tabular}{|l|c|c|c|c|}
\hline Operation & \multicolumn{5}{|c|}{ Distinct Operations } \\
\hline reverse $($ ) & \multicolumn{4}{|c|}{1} \\
\hline & \multicolumn{4}{|c|}{ Distinct Operations per Alphabet } \\
\hline & $\{\mathrm{A}, \mathrm{B}\}$ & $\{\mathrm{A}, \mathrm{B}, \mathrm{C}\}$ & $\{\mathrm{A}, \mathrm{B}, \mathrm{C}, \mathrm{D}\}$ & $\{\mathrm{A}, \mathrm{B}, \mathrm{C}, \mathrm{D}, \mathrm{E}\}$ \\
\hline concat$($ Literal $)$ & 2 & 3 & 4 & 5 \\
\hline concat $($ Simple $)$ & 1 & 1 & 1 & 1 \\
\hline concat $($ Complex $)$ & 1 & 1 & 1 & 1 \\
\hline & \multicolumn{4}{|c|}{ Distinct Operations per Length } \\
\hline & $\mathbf{1}$ & $\mathbf{2}$ & $\mathbf{3}$ & $\mathbf{4}$ \\
\hline delete $($ Same $)$ & 2 & 3 & 4 & 5 \\
\hline delete(Different $)$ & 1 & 3 & 6 & 10 \\
\hline Operation & \multicolumn{7}{|c|}{ Distinct Operations per Alphabet } \\
\hline & $\{$ A, B $\}$ & $\{$ A, B, C $\}$ & $\{$ A, B, C, D $\}$ & $\{$ A, B, C, D, E $\}$ \\
\hline replace $($ Same $)$ & 2 & 3 & 4 & 5 \\
\hline replace(Different $)$ & 2 & 6 & 9 & 16 \\
\hline
\end{tabular}

Table 5.4: The Number of Distinct Argument Combinations for String Operations

Unlike the other independent variables in the evaluation, the string operations variable is not evenly distributed through the Constraint Graphs. This is due to the different number of distinct operation configurations for the different chosen operations. This is further complicated by the different number of distinct operation argument configurations for different string alphabet and initial maximum string length values. These differences for the chosen operations is shown in Table 5.4. The table illustrates that the number of distinct replace and concat operations in a synthetic graph is dependent on the string alphabet. The number of distinct delete 
operations in turn is dependent on the initial maximum string length.

To ensure that any one string operations value does not skew the evaluation results, a data normalization process is applied the Collected Solved Constraint Data. This normalization applies integer weights to each result data row corresponding to the operations and argument configuration in the constraint solved to produce the row. For

example, the constraint contains(delete(replace $\left.\left.\left(s_{1}, \mathrm{~A}, \mathrm{~B}\right), 2,2\right), s_{4}\right)$ has the string operations $\{\mathrm{A}, \mathrm{B}, \mathrm{C}, \mathrm{D}, \mathrm{E}\}$ and initial maximum string length of 4 . The corresponding data row will be given a weight of 36 . This weight is the product of the weights for the two individual operation argument configurations, 3 for the replace operation with Different character arguments and 12 for the delete operation with Same integer arguments. This data normalization allows each of the distinct operation argument configurations to be included in a graph without skewing the result data.

\subsection{Accuracy}

The extent to which a solver can accurately represent the concrete values of concrete execution is the most important measure for determining its suitability in PSE. In this evaluation, two different measurements of model counting accuracy are taken by comparing the actual model count of the oracle solver to the model counts reported by the four automata-based solvers. This oracle is described in Section 5.3.1. Section 5.3.2 then explains the two different measurements of model counting accuracy. Additionally, Section 5.3.3 addresses the application of these metrics to the independent variable effect isolation experiments. 


\subsubsection{Oracle / ConcreteSolver}

In order to determine the accuracy of the model count reported by a solver, an actual model count is needed. In thes evaluation, ConcreteSolver described in Section 4.4.3 performs this task. Because ConcreteSolver performs its concrete operations and predicate for the Constraint Graphs through the same Solver interface used by the automata-based solvers, the model counts reported by the ConcreteSolver are an oracle of actual model counts for the Constraint Graphs. values returned by the concrete evaluation of actual string values provide the oracle for the automata models

which symbolically represent this exact concrete process. Just as with the automata models, the oracle records the before branch, the true branch, and the false branch model count. The model count prior to the branching predicate is recorded as the number of concrete string values, which reach the predicate and serve as the target string for the terminal predicate. The true branch model count is the number of concrete strings that evaluate to true. Similarly, the false branch model count is the number of concrete strings that evaluate to false. These three model count numbers are used to measure the accuracy of the solvers.

\subsubsection{Measurement}

The model counting accuracy of an automata models is measured in two ways: first as the frequency with which the more probable branch determined by the automata model agrees with the actual branch more likely to be executed and second as the model count difference between the actual model count and the count reported by the automata model. 


\section{Agreement}

When an automata model reports a branch as more probable to execute when the alternate branch is actually more likely to execute, this incorrect report of branch choice produces an invalid analysis as explained in Section 2.2.3. In this evaluation, the actual correct branch choice is provided by the oracle when the branch probabilities computed using Formula 2.1. To determine if the automata model agrees with the choice of more probable branch for a constraint, the true and false branch probabilities are compared using logical Formula 5.1 to determine agreement. This formula shows that agreement depends on either both the actual true branch probability $P_{t}^{A}$ and the automata model true branch probability $P_{t}$ being greater than or equal to their respective false branch probabilities, $P_{f}^{A}$ and $P_{f}$, or both false branches being more probable than the true branches. The frequency of these agreement results is recorded across all constraints for each automata model to measure the probability of an invalid analysis when using the model.

$$
\left(P_{t}^{A}>=P_{f}^{A} \wedge P_{t}>=P_{f}\right) \vee\left(P_{t}^{A}<P_{f}^{A} \wedge P_{t}<P_{f}\right) \Longrightarrow \text { Agreement }
$$

\section{Percentage difference}

Because automata models are representations of the concrete values satisfying a constraint, the actual numerical difference between the model count reported by the oracle and the model count reported by the automata model is not sufficient to measure accuracy. In PSE, model counts are used to determine the probabilities of taking one branch over another. For this reason, this evaluation uses the branch 
probabilities calculated using Formula 2.1 from the before branch and after branch model counts. The measurement of model counting accuracy (the branch percentage difference) is calculated from both the oracle and automata model branch probabilities using Formula 5.2. The formula calculates the percentage difference $P_{\text {diff }}$ as the absolute value of the difference between the actual branch probability $P_{b}^{A}$ and the automata model probability $P_{b}$. This percentage difference in branch probability measures the accuracy in the representation of concrete values by the symbolic string automata model.

$$
P_{\text {diff }}=\left|P_{b}^{A}-P_{b}\right|
$$

\subsubsection{Effects of Independent Variables}

The accuracy measurements of agreement and percentage difference are also applied to the results of the independent variable experiments. These additional experiments allow us to determine if there is a statistically significant effect for each independent variable on the model counting accuracy of each of the four automata models.

\subsection{Performance}

The second criteria used in evaluating the suitability of automata models in PSE is performance. Performance in this instance refers to the time required to perform the PSE analysis. A PSE analysis can be partitioned into the separate constraint solving and model counting phases for a constraint. While the time required for both of these 
parts of the analysis contribute to the overall required time, the time required by the constraint solving part has no influence on the time required by the model counting part and vice versa. Because of this separation, the data analysis can evaluate each performance metrics separately as well as their combination. This section is partitioned as follows: Section 5.4.1 explains the constraint solving performance metrics, Section 5.4.2 describes the model counting performance, and Section 5.4.3 details the combination of constraint solving and model counting performance.

\subsubsection{Constraint Solving Performance}

The constraint solving performance in a PSE analysis is determined by the time required to solve a string constraint for either the true or false branch of the constraint. This constraint solving time is the sum of the time required to initialize all the input string values to the constraint, the time required to model all of the intermediate operations and predicates for their symbolic string model arguments, and the time required to model either the true or false branch predicate for the two symbolic string model arguments.

\section{Measurement}

To measure the constraint solving time of a constraint, the following three result data columns are used: Cumulative Time, true Predicate Time, and false Predicate Time. The constraint solving time is calculated for either a true branch or a false branch and is the sum of the Cumulative Time and the respective branch predicate time. To compare the constraint solving time between the different automata models, the following is calculated from the constraint solving times of each model: average, median, variance, and standard deviation. These calculated values assess the ex- 
pected relative constraint solving performance for each automata model while also determining the consistency of this performance.

\subsubsection{Model Counting Performance}

The model counting performance in a string variable PSE analysis is the time required to determine the number of string values represented by the symbolic string model. This model counting process is described in Sections 3.1.1, 3.2.1, 3.3.2, and 3.4.3 for models in this evaluation, the Unbounded Automaton Model, the Bounded Automaton Model, the Aggregate Bounded Automata Model, and the Weighted Transition Aggregate Bounded Automata Model respectively.

\section{Measurement}

The experimental results for each constraint graph record the time in microsecond required to determine the model count from the model representing the true and false branches of the constraint. From these model count times, we compute the average, median, variance, and standard deviation of these times for each automata model. These measurements allow a relative performance comparison to be made between the

different automata models in terms of general performance and the consistency of such performances. Additionally, due to the almost identical model counting algorithms for each automata model, the relative performance between models will remain consistent for any alternative model counting algorithms.

\subsubsection{Combined Constraint Solving and Model Counting Performance}

The combined constraint solving and model counting performance is the time required to solve a constraint for either the true or false branch and to determine 


\begin{tabular}{|c|c|}
\hline Accuracy Measurement & Performance Measurement \\
\hline Percentage Difference & Constraint Solving Time \\
\hline Percentage Difference & Model Counting Time \\
\hline Percentage Difference & Combined Constraint Solving and Model Counting Time \\
\hline
\end{tabular}

Table 5.5: Accuracy vs Performance Comparison Analyses

the model count of the resulting symbolic string model. The combined constraint solving and model counting time is measured as the sum of the constraint solving time and the model counting time for either the true or false branch of a constraint. This additional measurement of performance is included in an attempt to evaluate the general relative performance of the four automata models in a PSE analysis.

\subsection{Comparison of Accuracy vs Performance}

In addition to individual analyses of the accuracy and performance of automata models in PSE, three additional analyses are performed to compare the accuracy and performance criteria for each automata-based solver. Each of the analyses listed in Table 5.5 compares the measurement of accuracy to the measurement of performance for true and false branches of all constraint in the Collected Solved Constraint Data.

The three analyses are performed to determine what relationship if any exists between the accuracy and performance metrics for each solver. The comparisons will provide guidance on which solvers are best when accuracy and performance are equally important for a PSE analysis. Additionally, the same three comparisons are performed for each of the experiments isolating independent variables. The additional comparisons allow the combined effects on accuracy and performance to be determined in their respective proportions. 


\subsection{Evaluation Environment}

The evaluation was conducted on a MacBook Pro $^{\mathrm{TM}^{\mathrm{w}}}$ with a 2 Ghz Intel Core $\mathrm{i} 7^{\mathrm{TM}}$ processor and $8 \mathrm{~GB}$ of RAM. The version $1.8 \mathrm{JVM}$ was used for the solving of constraints for each automata model and the oracle. The only change to the default JVM was a specification of maximum heap size which was set as 6 GB for all constraints solved. 


\section{CHAPTER 6}

\section{RESULTS AND CONCLUSIONS}

In this chapter we review the results of the evaluation described in Chapter 5. This chapter begins with Section 6.1 which examines the results of the evaluation with a focus on accuracy (Section 6.1.1), performance (Section 6.1.2), and the balance between accuracy and performance (Section 6.1.1). Section 6.2 offers guidance on the strengths and weaknesses observed for the solvers. Finally, Section 6.3 discusses the known threats to the validity of these results. Section 6.4 covers the conclusions which can be reached from the analysis. Section 6.5 explores possible directions for future work based on this analysis. Finally, section 6.6 concludes this paper with some final thoughts regarding the conducted analysis.

\subsection{Results}

The results of the evaluation of automata-based symbolic string model suitability are divided into the following three subsections: Section 6.1.1 reviews automata model accuracy, Section 6.1.2 reviews automata model performance, and Section 6.1.1 reviews the comparison of automata model accuracy versus model performance. 


\subsubsection{Accuracy}

Two different automata model accuracy measurements were recorded in the evaluation: the branch choice agreement described in Section 5.3.2 and the percentage difference described in Section 5.3.2. Section 6.1.1 reviews the branch choice agreement results. Section 6.1.1 reviews the percentage difference results.

\section{Branch Choice Agreement}

\begin{tabular}{|c|c|c|c|c|}
\hline Selection & Unbounded & Bounded & Aggregate & Weighted \\
\hline All & $84.6 \%$ & $99.3 \%$ & $99.3 \%$ & $99.8 \%$ \\
\hline
\end{tabular}

Table 6.1: Frequency of Branch Selection Agreement

Overall Table 6.1 displays the measured frequency of branch choice agreement for each of the automata models for all string constraints in the evaluated dataset. This table shows that the weighted automata model agrees with the constraint solving oracle more frequently than any other model. Unfortunately, this $99.8 \%$ branch choice agreement still leaves a $0.2 \%$ of string constraints where an invalid analysis still occurs. Later examination of the effects of the independent evaluation variables will demonstrate why these invalid analyses still occur for the weighted automata model.

The unbounded model chooses the incorrect branch with the most frequency where it disagrees for $15.4 \%$ of string constraints. This disagreement is $14.7 \%$ larger than the model with the second largest frequency of disagreement. This indicates that the use of an external maximum length, the only difference between the unbounded and bounded models, is a significant source of disagreement in unbounded automata models. The bounded and aggregate models both choose the correct predicate branch 
with $99.3 \%$ frequency which indicates that neither the subtractive collapse problems for bounded models nor the model count over-approximation for aggregate models are significant enough to cause a significant number of branch choice disagreements. The percentage difference measurements discussed in Section 6.1.1 will provide a more detailed comparison of the accuracy of these two models.

\begin{tabular}{|c|r|r|r|r|r|r|r|r|r|r|r|c|}
\hline & \multicolumn{2}{|c|}{ Unbounded } & \multicolumn{3}{|c|}{ Bounded } & \multicolumn{3}{c|}{ Aggregate } & \multicolumn{3}{c|}{ Weighted } \\
\hline Selection & Agree & \multicolumn{1}{|c|}{$\Delta$} & $r$ & Agree & \multicolumn{1}{c|}{$\Delta$} & $r$ & Agree & $\Delta$ & $r$ & Agree & $\Delta$ & $r$ \\
\hline$|\Sigma|=2$ & $82.6 \%$ & -2.0 & 0.02 & $98.9 \%$ & -0.4 & 0.01 & $98.8 \%$ & -0.5 & 0.02 & $99.6 \%$ & -0.2 & 0.01 \\
\hline$|\Sigma|=3$ & $84.5 \%$ & -0.1 & 0.00 & $99.4 \%$ & 0.1 & 0.00 & $99.3 \%$ & 0.0 & 0.00 & $99.9 \%$ & 0.1 & 0.01 \\
\hline$|\Sigma|=4$ & $85.8 \%$ & 1.2 & 0.01 & $99.4 \%$ & 0.1 & 0.01 & $99.5 \%$ & 0.2 & 0.01 & $99.8 \%$ & 0.0 & 0.00 \\
\hline$|\Sigma|=5$ & $85.7 \%$ & 1.1 & 0.01 & $99.6 \%$ & 0.3 & 0.02 & $99.6 \%$ & 0.3 & 0.02 & $99.9 \%$ & 0.1 & 0.01 \\
\hline$k=1$ & $84.2 \%$ & -0.4 & 0.00 & $98.8 \%$ & -0.5 & 0.02 & $98.9 \%$ & -0.4 & 0.02 & $99.6 \%$ & -0.2 & 0.01 \\
\hline$k=2$ & $84.7 \%$ & 0.1 & 0.00 & $99.3 \%$ & 0.0 & 0.00 & $99.3 \%$ & 0.0 & 0.00 & $99.7 \%$ & -0.1 & 0.01 \\
\hline$k=3$ & $84.7 \%$ & 0.1 & 0.00 & $99.5 \%$ & 0.2 & 0.01 & $99.5 \%$ & 0.2 & 0.01 & $99.9 \%$ & 0.1 & 0.01 \\
\hline$k=4$ & $84.9 \%$ & 0.3 & 0.00 & $99.7 \%$ & 0.4 & 0.02 & $99.6 \%$ & 0.3 & 0.02 & $100.0 \%$ & 0.2 & 0.02 \\
\hline Literal & $82.7 \%$ & -1.9 & 0.02 & $99.6 \%$ & 0.3 & 0.02 & $99.6 \%$ & 0.3 & 0.02 & $99.6 \%$ & -0.2 & 0.02 \\
\hline Simple & $86.5 \%$ & 1.9 & 0.02 & $99.0 \%$ & -0.3 & 0.01 & $99.0 \%$ & -0.3 & 0.01 & $99.9 \%$ & 0.1 & 0.01 \\
\hline Complex & $84.7 \%$ & 0.1 & 0.00 & $99.4 \%$ & 0.1 & 0.01 & $99.4 \%$ & 0.1 & 0.01 & $99.8 \%$ & 0.0 & 0.00 \\
\hline
\end{tabular}

Table 6.2: Frequency of Branch Selection Agreement For Different Initial String Characteristics

Initial String Type Table 6.2 displays the measured frequency of branch choice agreement for each of the automata models for the string constraints in the evaluated dataset having the specified initial string characteristic. The $\Delta$ column for each automata model provides the difference between the overall agreement and this specific data subset. The $r$ column for each automata model is the correlation coefficient which is a measure of the size of the effect of the independent variables. The guidelines for effect size of $r$ are as follows: a small effect size is 0.1 , a medium effect size is 0.3 , and a large effect size is 0.5 . This table focuses on the three following initial string characteristics: alphabet size, initial string length, and initial string type. 
The effects of both the alphabet size and the initial string length on the branch choice agreement are similar for each of the four automata models. None of the effect sizes measured by the $r$ correlation coefficient indicate a significant effect with the largest effect sizes measured at 0.02 which is well below the threshold for even a small effect size at 0.10 . A trend seen from the $\Delta$ for each of the automata models is the increasing frequency of branch choice agreement as both alphabet size and initial string length increase. Extrapolating from this initial trend for each model, we expect the branch choice agreement frequency to increase for each larger alphabet size and initial string length.

Each of the initial string types has a minimal effect on the frequency of branch choice agreement where 0.02 is the largest measured effect size for a string type. This 0.02 effect size is the result of disagreement for a literal initial strings using either unbounded or weighted models. The only other string type with larger disagreement than overall is for the simple type where both the bounded and aggregate models measure a 0.01 effect size for the increased disagreement frequency.

String Operations Table 6.3 displays the measured frequency of branch choice agreement for each of the automata models for the string constraints in the evaluated dataset which use the specified string operation. The $\Delta$ column provides the difference between the overall and data subset percentage difference. The $r$ column shows the effect size for the independent variable. The table shows results for the following four string operations: concat, delete, replace, and reverse. The $\exists$ prefix for an operation indicates a data subset which includes at least one instance of the operation in the constraint. The $\forall$ prefix for an operation indicates a data subset which includes only instances of the operation in the constraint.

The branch agreement frequency of the unbounded automata model is significantly 


\begin{tabular}{|c|c|c|c|c|c|c|c|c|c|c|c|c|}
\hline & \multicolumn{3}{|c|}{ Unbounded } & \multicolumn{3}{|c|}{ Bounded } & \multicolumn{3}{|c|}{ Aggregate } & \multicolumn{3}{|c|}{ Weighted } \\
\hline $\mathbf{S}$ & gree & $\Delta$ & $r$ & ree & $\Delta$ & $r$ & gree & $\Delta$ & $r$ & gree & $\Delta$ & $r$ \\
\hline$\exists \mathrm{cc}$ & & & & & 3 & & & & & & 1 & \\
\hline concat $(L)$ & & 6 & & & 2 & & & 2 & & & .1 & 0.01 \\
\hline con & 格 & 0. & & 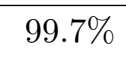 & 0.4 & & 年 & 0.4 & & $9.9 \%$ & .1 & 0.0 \\
\hline con & $.4 \%$ & 0.4 & & (6.0 & 0.3 & & 年 & 0.4 & & $9.9 \%$ & .1 & 0.0 \\
\hline$\forall$ concat & $.1 \%$ & . & & $99.6 \%$ & 0.3 & 0.0 & $99.9 \%$ & 0.6 & & $9.9 \%$ & 0.1 & 0.01 \\
\hline$\forall \operatorname{concat}(L)$ & $.7 \%$ & -13.9 & & 0.1 & 7 & 0.00 & $\%$ & 7 & 0.06 & $00.0 \%$ & .2 & .0 \\
\hline$\forall$ conc & $.5 \%$ & 0.1 & 019 & 0057 & 0.2 & 0.01 & $99.5 \%$ & 2 & 001 & $\%$ & -0.3 & 0 \\
\hline$\forall$ concat $(C)$ & $2.2 \%$ & 12.4 & & $98.8 \%$ & -0.5 & 0.00 & 8 & 4 & & $.7 \%$ & -0.1 & 0.01 \\
\hline$\exists \mathrm{de}$ & 0 & 7.1 & & & 0.0 & & & .0 & & $\%$ & -0.1 & 0.01 \\
\hline$\exists \mathrm{d}$ & & & & & & & & & & & 0.0 & \\
\hline$\exists \mathrm{del}$ & $95.5 \%$ & & & $99.1 \%$ & 2 & & & & & $\%$ & -0.1 & 0.01 \\
\hline$\forall$ delete & $99.5 \%$ & 14 & & 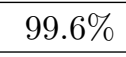 & 0.3 & & $99.5 \%$ & & & $99.8 \%$ & 0.0 & .00 \\
\hline$\forall \operatorname{delete}(s)$ & $99.5 \%$ & 14.9 & 0. & $99.8 \%$ & 0.5 & 0.0 & $99.8 \%$ & 0.5 & 0.0 & $99.8 \%$ & 0.0 & 0.00 \\
\hline$\forall$ delete $(d)$ & $99.5 \%$ & 14.9 & 028 & $9.5 \%$ & 0.2 & 0. & $99.5 \%$ & 0.2 & 0. & $99.8 \%$ & 0.0 & 0.00 \\
\hline$\exists$ replace & $87.6 \%$ & 3.0 & & $8.9 \%$ & -0.4 & 00 & $98.9 \%$ & 0.8 & 0.02 & $99.7 \%$ & -0.1 & 0. \\
\hline replace $(s)$ & 89.0 & 4.4 & & 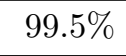 & 2 & 0.01 & $99.5 \%$ & 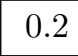 & & $9.8 \%$ & .0 & 0 \\
\hline$\exists$ replace $(d)$ & 87.5 & 2.9 & 0.0 & $\%$ & -1.2 & 0. & $\%$ & -1.2 & 0 & $99.6 \%$ & -0.2 & 0.02 \\
\hline$\forall$ replace & $97.6 \%$ & 13.0 & 0. & $97.9 \%$ & -1.4 & 0.0 & $97.9 \%$ & -1.4 & 0.1 & $99.6 \%$ & -0.2 & 0.02 \\
\hline$\forall$ replace $(s)$ & $99.8 \%$ & 15.2 & 0.2 & $99.8 \%$ & 0.5 & 0.0 & $99.8 \%$ & 0.5 & 0.0 & $99.8 \%$ & 0.0 & 0.00 \\
\hline$\forall$ replace $(d)$ & $96.4 \%$ & 11.8 & 0.2 & $96.9 \%$ & -2.4 & 0.09 & $96.9 \%$ & -2.4 & 0.09 & $99.4 \%$ & -0.4 & 0.03 \\
\hline$\exists$ reverse & $89.0 \%$ & 4.4 & 0.1 & $99.5 \%$ & 0.2 & 0.0 & $99.4 \%$ & 0.1 & 0.0 & $99.8 \%$ & 0.0 & 0.00 \\
\hline$\forall$ reverse & $99.9 \%$ & 15.3 & 0.29 & $99.9 \%$ & 0.6 & 0.05 & $99.9 \%$ & 0.6 & 0.05 & $99.9 \%$ & 0.1 & 0.01 \\
\hline
\end{tabular}

Table 6.3: Frequency of Branch Selection Agreement For Different Operations

affected by the concat operation. The $\Delta$ for each concat data subset is negative and each has an effect size greater than 0.10 which is the small effect size threshold. Because each of the exclusive data subsets for the delete, replace, and reverse operations have both positive $\Delta$ values and effect sizes just short of the medium effect size threshold of 0.30 , we can confirm that branch choice disagreement for the unbounded model is the primarily the result of additive string operations such as concat. 
The bounded and aggregate automata models have almost equivalent branch choice agreement for the different string operation data subsets. Both automata models have the largest disagreement due to replace operations where the string constraints which include only replace operations with different character arguments. The effect size for this data subset is 0.09 , only slightly less than the threshold of small effect sizes at 0.10 . The only significant difference between the agreement frequency for unbounded and aggregate models is seen for the exclusive concat operations with complex arguments where the bounded model has a negative $\Delta$ and the aggregate model has a positive delta.

The effect of string operations on the agreement frequency for weighted automata models is negligible where the maximum effect size is 0.03 . While there are some negative $\Delta$ values for the different string operation data subsets, the smallest agreement percentage of $99.4 \%$ indicates the lack of significant effects on weighted automata model agreement frequency due to the operations in a string constraint.

\begin{tabular}{|c|r|r|c|r|r|r|r|r|r|r|r|c|}
\hline & \multicolumn{3}{|c|}{ Unbounded } & \multicolumn{3}{|c|}{ Bounded } & \multicolumn{3}{c|}{ Aggregate } & \multicolumn{3}{c|}{ Weighted } \\
\hline Selection & Agree & \multicolumn{1}{c|}{$\Delta$} & \multicolumn{1}{c|}{$r$} & Agree & \multicolumn{1}{c|}{$\Delta$} & $r$ & Agree & $\Delta$ & $r$ & Agree & \multicolumn{1}{c|}{$\Delta$} & $r$ \\
\hline contains & $98.9 \%$ & 14.3 & 0.22 & $99.2 \%$ & -0.1 & 0.01 & $99.2 \%$ & -0.1 & 0.01 & $99.6 \%$ & -0.2 & 0.02 \\
\hline contains $(L)$ & $97.6 \%$ & 13.0 & 0.13 & $98.0 \%$ & -1.3 & 0.05 & $98.1 \%$ & -1.2 & 0.05 & $99.0 \%$ & -0.8 & 0.05 \\
\hline contains $(S)$ & $100.0 \%$ & 15.4 & 0.14 & $100.0 \%$ & 0.7 & 0.03 & $100.0 \%$ & 0.7 & 0.03 & $100.0 \%$ & 0.2 & 0.01 \\
\hline contains $(C)$ & $99.1 \%$ & 14.5 & 0.17 & $99.6 \%$ & 0.3 & 0.01 & $99.7 \%$ & 0.4 & 0.02 & $99.9 \%$ & 0.1 & 0.01 \\
\hline equals & $70.4 \%$ & -14.2 & 0.17 & $99.5 \%$ & 0.2 & 0.01 & $99.4 \%$ & 0.1 & 0.01 & $100.0 \%$ & 0.2 & 0.03 \\
\hline equals $(L)$ & $98.7 \%$ & 14.1 & 0.14 & $98.6 \%$ & -0.7 & 0.03 & $98.5 \%$ & -0.8 & 0.03 & $100.0 \%$ & 0.2 & 0.02 \\
\hline equals $(S)$ & $45.3 \%$ & -39.3 & 0.30 & $100.0 \%$ & 0.7 & 0.03 & $100.0 \%$ & 0.7 & 0.03 & $100.0 \%$ & 0.2 & 0.01 \\
\hline equals $(C)$ & $63.0 \%$ & -21.6 & 0.21 & $99.8 \%$ & 0.5 & 0.02 & $99.8 \%$ & 0.5 & 0.02 & $100.0 \%$ & 0.2 & 0.02 \\
\hline
\end{tabular}

Table 6.4: Frequency of Branch Selection Agreement For contains and equals Predicates

String Predicates Table 6.4 displays the measured frequency of branch choice agreement for each of the automata models for the string constraints in the evaluated 
dataset which use the specified string predicate. The $\Delta$ column provides the difference between the overall and data subset percentage difference. The $r$ column shows the effect size for the independent variable. The table shows results for the contains and equals string predicates.

The unbounded automata model shows very poor agreement accuracy for the equals predicates using both simple and complex types of string arguments. The 0.30 correlation coefficient for the equals predicate using simple arguments meets the threshold of a medium effect size $(r \geq 0.30)$. This effect of disagreement along with the 0.21 effect size for disagreement of equals predicates using complex arguments account for nearly all of the branch choice disagreement for unbounded automata models.

Again the bounded and aggregate automata models have very similar frequencies of branch choice agreement for the different predicate data subsets. Both of these automata models have larger disagreement due toe predicates using a literal string type argument when compared to the positive $\Delta$ values for the other argument specific predicate data subsets. However, neither of these disagreements is as significant as the operation data subsets since the 0.05 and 0.03 effect sizes are significantly less than the 0.09 effect size maximum from the operation data subsets.

The predicate data subset for the weighted automata model is the most significant of all the data subsets for the model. Every variation of the equals predicate has $100 \%$ branch choice agreement, demonstrating that all branch choice disagreements arise from contains predicates. Specifically, the contains predicate for literal string type arguments is the only contains data subset with a negative $\Delta$ with an effect size of 0.05 , the largest effect size of any data subset for the weighted automata model. 


\section{Model Count Percentage Difference}

\begin{tabular}{|c|c|c|c|c|}
\hline Selection & Unbounded & Bounded & Aggregate & Weighted \\
\hline Average & 9.93 & 1.02 & 0.95 & 0.40 \\
\hline Standard Deviation & 27.12 & 5.54 & 5.57 & 4.76 \\
\hline
\end{tabular}

Table 6.5: Model Counting Percentage Difference

Overall Table 6.5 displays the metrics for the difference between model and oracle branch probability for each of the automata models for all string constraints in the evaluated dataset. The model with the largest average percentage difference is the unbounded automata model at 9.93 which is nearly ten times the second largest percentage difference of the bounded model at 1.02. The bounded and aggregate are only separated by an average difference of 0.07 . The weighted model is clearly the most accurate automata model with an average percentage difference of only 0.40 , less than half the difference of the aggregate model. While each of the bounded, aggregate, and weighted automata models have average differences of 1.02 or less, the standard deviations for each of these models is significantly higher ranging from 5.57 for the aggregate model to 4.76 for the weighted model. These larger standard deviations indicate that the majority of constraints had no or very small percentage differences. The standard deviation of the unbounded automata model at 27.12 indicate the magnitude of the problem with inaccuracy for the model.

\begin{tabular}{|c|r|r|c|c|c|c|c|c|c|c|c|c|}
\hline & \multicolumn{3}{|c|}{ Unbounded } & \multicolumn{3}{c|}{ Bounded } & \multicolumn{3}{c|}{ Aggregate } & \multicolumn{3}{c|}{ Weighted } \\
\hline Selection & Avg & $\Delta$ & $d$ & Avg & \multicolumn{1}{|c|}{$\Delta$} & $d$ & Avg & $\Delta$ & $d$ & Avg & $\Delta$ & $d$ \\
\hline true Branches & 16.28 & 6.35 & 0.19 & 0.99 & -0.03 & 0.01 & 0.88 & -0.07 & 0.02 & 0.05 & -0.35 & 0.41 \\
\hline false Branches & 3.57 & -6.36 & 0.40 & 1.06 & 0.04 & 0.01 & 1.02 & 0.07 & 0.01 & 0.75 & 0.35 & 0.05 \\
\hline
\end{tabular}

Table 6.6: Model Counting Percentage Difference For true and false Branches 
true or false Predicate Branch Table 6.6 shows the metrics for the difference between model and oracle branch probability for each of the automata models for the string constraints in the evaluated dataset for either only the true branch or only the false branch. The $\Delta$ column provides the difference between the overall percentage difference and the percentage difference of the specific data subset. The $d$ column uses Cohen's $d$ measurment of effect size which has the following guidelines: a very small effect is 0.01 , a small effect is 0.2 , a medium effect is 0.5 , a large effect is 0.8 , a very large effect is 1.2 , and a huge effect is 2.0 .

The unbounded automata model has a clear accuracy deficiency when calculating the probability of the true constraint branch shown by the effect size of 0.19 which is just short of the small effect size threshold of 0.20 . This increased difference in true predicate branches is explained by the inaccuracy of full match predicates for unbounded models seen earlier in agreement frequency as well as shown later when examining percentage differences for predicated data subsets. Each of the bounded, aggregate, and weighted automata models average larger percentage differences for false branches than for true branches. While the bounded and aggregate models have very small effect sizes of 0.01 due to the false branchs, the weighted model has a larger effect size of 0.05 although that still falls well short of the small effect size threshold of 0.20 .

Initial String Type Table 6.7 shows the metrics for the difference between model and oracle branch probability for each of the automata models for the string constraints in the evaluated dataset having the specified initial string characteristic. The $\Delta$ column provides the difference between the overall and data subset percentage difference. The $d$ column shows the effect size for the independent variable. This table focuses on the three following initial string characteristics: alphabet size, initial 


\begin{tabular}{|c|r|r|c|c|c|c|c|c|c|c|c|c|}
\hline & \multicolumn{3}{|c|}{ Unbounded } & \multicolumn{3}{c|}{ Bounded } & \multicolumn{3}{c|}{ Aggregate } & \multicolumn{3}{c|}{ Weighted } \\
\hline Selection & Avg & \multicolumn{1}{|c|}{$\Delta$} & $d$ & Avg & $\Delta$ & $d$ & Avg & $\Delta$ & $d$ & Avg & $\Delta$ & $d$ \\
\hline$|\Sigma|=2$ & 10.80 & 0.87 & 0.03 & 1.82 & 0.80 & 0.09 & 1.72 & 0.77 & 0.09 & 0.97 & 0.57 & 0.07 \\
\hline$|\Sigma|=3$ & 10.01 & 0.08 & 0.00 & 0.87 & -0.15 & 0.03 & 0.82 & -0.13 & 0.03 & 0.27 & -0.13 & 0.04 \\
\hline$|\Sigma|=4$ & 9.55 & -0.38 & 0.01 & 0.79 & -0.23 & 0.05 & 0.71 & -0.24 & 0.06 & 0.25 & -0.15 & 0.04 \\
\hline$|\Sigma|=5$ & 9.34 & -0.59 & 0.02 & 0.59 & -0.43 & 0.13 & 0.56 & -0.39 & 0.11 & 0.12 & -0.28 & 0.14 \\
\hline$k=1$ & 12.95 & 3.02 & 0.10 & 1.41 & 0.39 & 0.05 & 1.26 & 0.31 & 0.04 & 0.91 & 0.51 & 0.06 \\
\hline$k=2$ & 8.70 & -1.23 & 0.05 & 1.18 & 0.16 & 0.03 & 1.10 & 0.15 & 0.03 & 0.44 & 0.04 & 0.01 \\
\hline$k=3$ & 8.93 & -1.00 & 0.04 & 0.83 & -0.19 & 0.05 & 0.79 & -0.16 & 0.04 & 0.14 & -0.26 & 0.12 \\
\hline$k=4$ & 9.11 & -0.82 & 0.03 & 0.67 & -0.35 & 0.10 & 0.66 & -0.29 & 0.08 & 0.11 & -0.29 & 0.15 \\
\hline Literal & 10.12 & 0.19 & 0.01 & 0.42 & -0.60 & 0.11 & 0.41 & -0.54 & 0.10 & 0.37 & -0.03 & 0.01 \\
\hline Simple & 9.30 & -0.63 & 0.03 & 1.50 & 0.48 & 0.10 & 1.31 & 0.36 & 0.07 & 0.40 & 0.00 & 0.00 \\
\hline Complex & 10.33 & 0.40 & 0.01 & 1.14 & 0.12 & 0.02 & 1.12 & 0.17 & 0.03 & 0.42 & 0.02 & 0.00 \\
\hline
\end{tabular}

Table 6.7: Model Counting Percentage Difference For Different Initial String Characteristics

string length, and initial string type.

Each of the four automata models demonstrate increasing branch probability accuracy as either the alphabet size or the initial string length increase. This trend of increasing branch probability accuracy is a stronger indication than the branch agreement frequency results for determining that larger alphabet sizes and initial string lengths will produce more accurate automata models.

The literal initial string type only increased percentage difference for the unbounded automata model and only with the very small effect size of 0.01. The simple initial string type increased the percentage difference for both the bounded and aggregate automata models with 0.10 and 0.07 effect sizes respectively. While this is half and less than half of the small effect size threshold, these effect sizes are some of the largest out of the independent variable data subsets for both of these automata models. The complex initial string type has an increased percentage difference for each of the four automata models, but not with any significant effect sizes. 


\begin{tabular}{|c|c|c|c|c|c|c|c|c|c|c|c|c|}
\hline & \multicolumn{3}{|c|}{ Unbounded } & \multicolumn{3}{|c|}{ Bounded } & \multicolumn{3}{|c|}{ Aggregate } & \multicolumn{3}{|c|}{ Weighted } \\
\hline Selection & Avg & $\Delta$ & $d$ & Avg & $\Delta$ & $d$ & Avg & $\Delta$ & $d$ & Avg & $\Delta$ & $d$ \\
\hline$\exists$ concat & 14.94 & 5.01 & 0.15 & 0.94 & -0.08 & 0.02 & 0.81 & -0.14 & 0.03 & 0.39 & -0.01 & 0.00 \\
\hline$\exists \operatorname{concat}(L)$ & 14.38 & 4.45 & 0.14 & 0.82 & -0.20 & 0.03 & 0.85 & \begin{tabular}{|l|}
-0.10 \\
\end{tabular} & 0.02 & 0.41 & 0.01 & 0.00 \\
\hline$\exists \operatorname{concat}(S)$ & 15.51 & 5.58 & 7 & 1.24 & 0.22 & 0.04 & .86 & \begin{tabular}{|l|}
-0.09 \\
\end{tabular} & .02 & 0.48 & 0.08 & 0.02 \\
\hline$\exists \operatorname{concat}(C)$ & 16.04 & 6.11 & 0.18 & 0.82 & -0.20 & 0.04 & 0.72 & -0.23 & 0.04 & 0.41 & 0.01 & 0.00 \\
\hline$\forall$ concat & 17.98 & 8.05 & 0.22 & 0.79 & -0.23 & 0.04 & 0.66 & -0.29 & 0.05 & 0.60 & 0.20 & 0.03 \\
\hline$\forall \operatorname{concat}(L)$ & 16.94 & 7.01 & .19 & 0.52 & -0.50 & 0.07 & 0.52 & -0.43 & 0.06 & 0.52 & 0.12 & 0.02 \\
\hline$\forall \operatorname{concat}(S)$ & 17.53 & 7.60 & 0.22 & 1.35 & 0.33 & 0.06 & 0.79 & -0.16 & 0.03 & 0.73 & 0.33 & 0.06 \\
\hline$\forall$ concat $(C)$ & 17.92 & 7.99 & 0.22 & 0.67 & -0.35 & 0.07 & 0.48 & \begin{tabular}{|l|}
-0.47 \\
\end{tabular} & 0.09 & 0.44 & 0.04 & 0.01 \\
\hline$\exists$ delete & 5.66 & -4.27 & 0.21 & 0.95 & -0.07 & 0.01 & 0.91 & -0.04 & 0.01 & 0.42 & 0.02 & 0.00 \\
\hline$\exists$ delete $(s)$ & 7.23 & -2.70 & 0.11 & 0.67 & -0.35 & 0.07 & 0.59 & -0.36 & 0.08 & 0.33 & -0.07 & 0.02 \\
\hline$\exists$ delete $(d)$ & 3.46 & -6.47 & 0.45 & 1.22 & 0.20 & 0.03 & 1.24 & 0.29 & 0.05 & 0.51 & 0.11 & 0.02 \\
\hline$\forall$ delete & 0.95 & -8.98 & 1.35 & 0.70 & -0.32 & 0.07 & 0.71 & -0.24 & 0.05 & 0.35 & -0.05 & 0.01 \\
\hline$\forall$ delete $(s)$ & 1.27 & -8.66 & 0.90 & 0.29 & -0.73 & 0.21 & 0.29 & -0.66 & 0.19 & 0.29 & -0.11 & 0.03 \\
\hline$\forall$ delete $(d)$ & 0.73 & -9.20 & 1.88 & 0.73 & -0.29 & 0.06 & 0.75 & -0.20 & 0.04 & 0.36 & -0.04 & 0.01 \\
\hline$\exists$ replace & 8.27 & -1.66 & 0.07 & 1.37 & 0.35 & 0.06 & 1.36 & 0.41 & 0.07 & 0.36 & -0.04 & 0.01 \\
\hline$\exists \operatorname{replace}(s)$ & 7.54 & -2.39 & 0.10 & 0.80 & -0.22 & 0.04 & 0.71 & -0.24 & 0.05 & 0.32 & -0.08 & 0.02 \\
\hline$\exists$ replace $(d)$ & 8.48 & -1.45 & 0.06 & 2.07 & 1.05 & 0.15 & 2.14 & 1.19 & 0.17 & 0.40 & 0.00 & 0.00 \\
\hline$\forall$ replace & 3.82 & -6.11 & 0.41 & 1.88 & 0.86 & 0.12 & 1.88 & 0.93 & 0.13 & 0.41 & 0.01 & 0.00 \\
\hline$\forall$ replace $(s)$ & 2.17 & -7.76 & 0.58 & 0.25 & -0.77 & 0.24 & 0.25 & -0.70 & 0.21 & 0.25 & -0.15 & 0.05 \\
\hline$\forall$ replace $(d)$ & 4.64 & -5.29 & 0.34 & 2.66 & 1.64 & 0.19 & 2.66 & 1.71 & 0.20 & 0.51 & 0.11 & 0.02 \\
\hline$\exists$ reverse & 7.51 & -2.42 & 0.10 & 0.81 & -0.21 & 0.04 & 0.72 & -0.23 & 0.05 & 0.35 & -0.05 & 0.01 \\
\hline$\forall$ reverse & 2.25 & -7.68 & 0.56 & 0.24 & -0.78 & 0.26 & 0.24 & \begin{tabular}{|l|}
-0.71 \\
\end{tabular} & 0.23 & 0.24 & -0.16 & 0.05 \\
\hline
\end{tabular}

Table 6.8: Model Counting Percentage Difference For Different String Operations

String Operations Table 6.8 shows the metrics for the difference between model and oracle branch probability for each of the automata models for the string constraints in the evaluated dataset which uses the specified string operation. The $\Delta$ column provides the difference between the overall and data subset percentage difference. The $d$ column shows the effect size for the independent variable. The table shows results for the following four string operations: concat, delete, replace, and reverse.

The unbounded automata model is significantly affected by additive string op- 
erations as demonstrated by the concat data subsets where the effect sizes range from 0.14 to 0.22 . These results indicate that over-approximation of the solution sets seen in unbounded automata models produces greater inaccuracy than the different collapse problems due to subtractive and substitutive operations. From these unbouned percentage difference results, we can see that the percentage difference due to subtractive collapse is significantly less than the difference due to substitutive collapse. Specifically, the 0.73 percentage difference of delete operation with different index arguments provides a positive 1.88 effect size which is closer to the huge effect size threshold at 2.0 than the large effect size threshold at 1.2. Comparing this to the replace operation with different character arguments which has a 4.64 percentage difference and only a 0.34 positive effect size. This comparison clearly shows that collapses due to substitutive operations such as replace are a more signifigant source of inaccuracy for automata-based symbolic string models.

The bounded and aggregate automata models both have the largest difference in branch probability due to the replace string operation. The two data subsets for replace operations using different character arguments produce effect sizes of 0.19 and 0.20 for the bounded and aggregate models respectively. These results indicate the small but significant effect that substitutive collapses have on the branch probability and therefore the model accuracy. As with the agreement frequency, this measurement of percentage difference also indicates less accuracy for a bounded model for concat operations using simple string type arguments.

The weighted automata model has only insignificant effect sizes ranging from 0.00 to 0.06 for each of the operation data subsets. While the 0.06 effect size falls well below the small effect size threshold of 0.20 , it is the largest effect size of all the operation data subsets indicating minor inaccuracies in branch probabilities due to 
additive operations for weighted automata models.

\begin{tabular}{|c|r|r|c|c|c|c|c|c|c|c|c|c|}
\hline & \multicolumn{3}{|c|}{ Unbounded } & \multicolumn{3}{c|}{ Bounded } & \multicolumn{3}{c|}{ Aggregate } & \multicolumn{3}{c|}{ Weighted } \\
\hline Selection & Avg & $\Delta$ & $d$ & Avg & $\Delta$ & $d$ & Avg & $\Delta$ & $d$ & Avg & $\Delta$ & $d$ \\
\hline contains & 3.27 & -6.66 & 0.46 & 1.26 & 0.24 & 0.03 & 1.19 & 0.24 & 0.03 & 0.75 & 0.35 & 0.05 \\
\hline contains $(L)$ & 2.19 & -7.74 & 0.86 & 1.92 & 0.90 & 0.10 & 1.81 & 0.86 & 0.10 & 1.18 & 0.78 & 0.09 \\
\hline contains $(S)$ & 1.04 & -8.89 & 1.04 & 1.04 & 0.02 & 0.00 & 1.04 & 0.09 & 0.01 & 1.04 & 0.64 & 0.08 \\
\hline contains $(C)$ & 5.49 & -4.44 & 0.22 & 0.87 & -0.15 & 0.04 & 0.79 & -0.16 & 0.04 & 0.22 & -0.18 & 0.07 \\
\hline equals & 16.58 & 6.65 & 0.19 & 0.78 & -0.24 & 0.07 & 0.71 & -0.24 & 0.07 & 0.05 & -0.35 & 0.41 \\
\hline equals $(L)$ & 1.04 & -8.89 & 2.23 & 0.91 & -0.11 & 0.03 & 0.93 & -0.02 & 0.00 & 0.09 & -0.31 & 0.27 \\
\hline equals $(S)$ & 24.52 & 14.59 & 0.36 & 0.61 & -0.41 & 0.14 & 0.45 & -0.50 & 0.18 & 0.03 & -0.37 & 0.55 \\
\hline equals $(C)$ & 24.25 & 14.32 & 0.37 & 0.78 & -0.24 & 0.07 & 0.69 & -0.26 & 0.08 & 0.04 & -0.36 & 0.57 \\
\hline
\end{tabular}

Table 6.9: Model Counting Percentage Difference For contains and equals Predicates

String Predicates Table 6.9 shows the metrics for the difference between model and oracle branch probability for each of the automata models for the string constraints in the evaluated dataset which uses the specified string predicates. The $\Delta$ column provides the difference between the overall and data subset percentage difference. The $d$ column shows the effect size for the independent variable. The $r$ column shows the effect size for the independent variable. The table shows results for the contains and equals string predicates.

The unbounded automata model has the most significant difference to branch probability due to equals predicates for both the simple and complex types of arguments. The effect sizes of 0.36 and 0.37 are the largest effect sizes for an unbounded model due to an increase in percentage difference, demonstrating that full match predicates are the largest source of inaccuracy for unbounded models. This is consistent with the results for the frequency of branch choice agreement.

Each of the bounded, aggregate, and weighted automata models have increased percentage differences for the contains predicates using literal and simple arguments. 
For each of these three models, the contains predicate with literal arguments is a significant source of percentage difference for the model where it is the second largest effect size for both the bounded and aggregate models and is the largest effect size of any of the independent variable data subsets for the weighted model. The weighted model is also significantly affected by the contains predicate with simple arguments with the second largest effect size of weighted models.

\subsubsection{Performance}

The findings of the evaluation for solver suitability based upon performance criteria appear in the following three subsections: cumulative constraint solving time in Section 6.1.2, model counting time in Section 6.1.2, and combined cumulative constraint solving and model counting time in Section 6.1.2.

\section{Cumulative Constraint Solving Time}

Figure 6.1 shows the boxplot for the constraint solving time of each automata model where the $\mathrm{y}$-axis is in microseconds and is on a logarithmic scale. The figure shows that the constraint solving times for the unbounded and bounded automata models are very similar where the solving time for bounded models varies slightly more than unbounded models. The aggregate automata model requires more time to solve string constraints than either the unbounded or the bounded models which is to be expected due to the aggregate model containing a sequence of FSAs instead of a single FSA. Finally, the weighted automata model has significantly worse constraint solving performance than any of the other three automata models. This performance difference is due to the complexity added by weighted transitions when simulating string operations and predicates. 


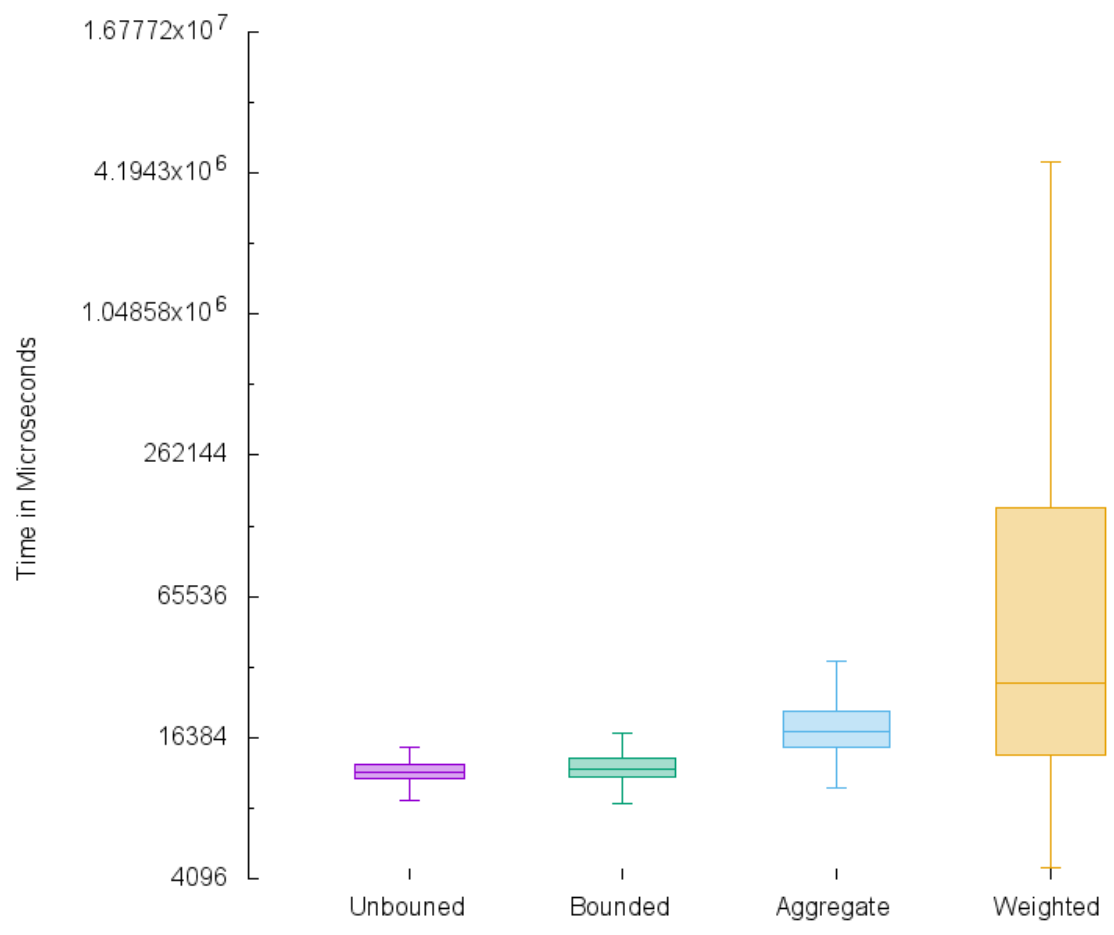

Figure 6.1: Boxplots of Constraint Solving Times

\section{Model Counting Time}

Figure 6.2 shows the boxplots for both the model counting times and the constraint solving times of each automata model where the y-axis is in microseconds and is on a logarithmic scale. The boxplots for the model counting times (labeled MC) of each of the four automata models shows that model counting performance is nearly equivalent between models. The box plots for constraint solving time (labeled S) in the same plot shows the extreme difference between model counting and constraint solving times, demonstrating the practical irrelevance of model counting time to the overall analysis time in a quantitative string analysis. 


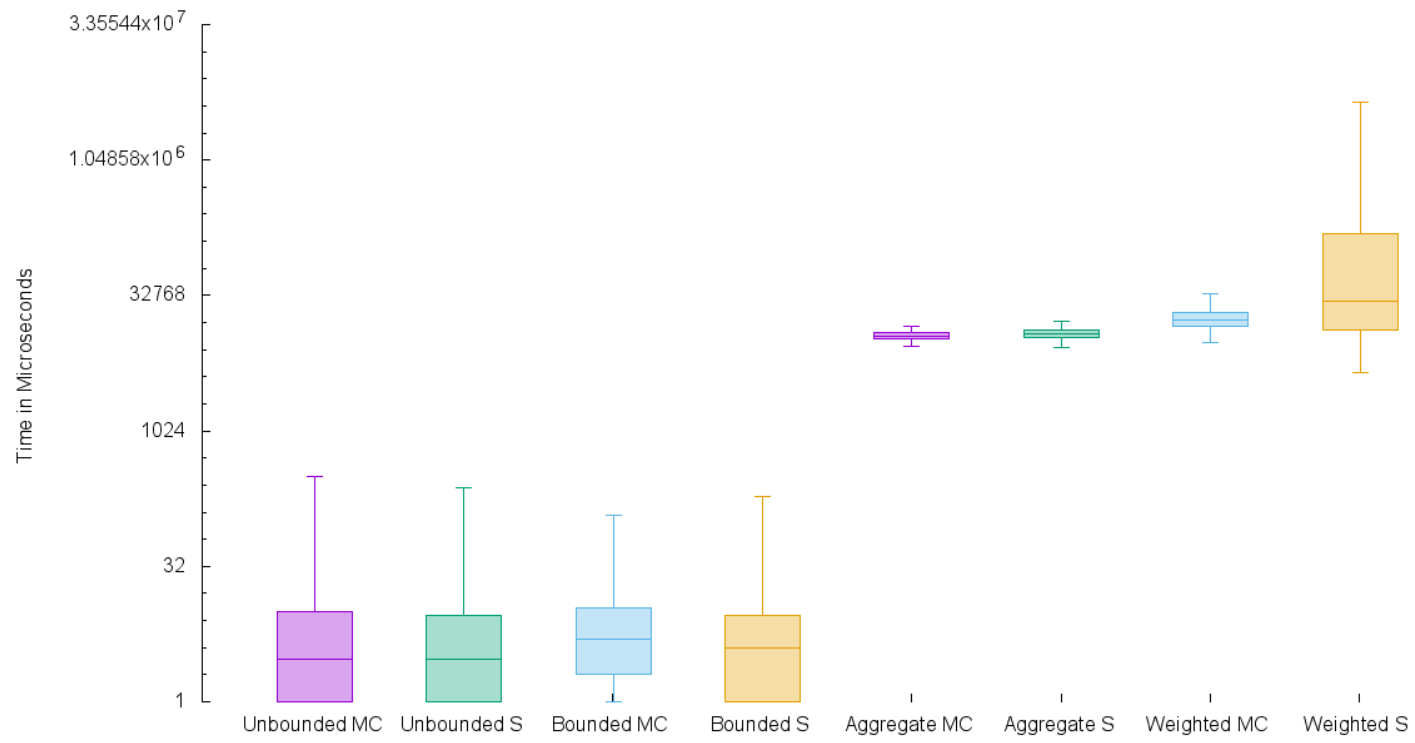

Figure 6.2: Boxplots of Model Counting Times and Constraint Solving Times

\section{Combined Model Counting and Constraint Solving Time}

Figure 6.3 shows the boxplot for the combined constraint solving and model counting time of each automata model where the y-axis is in microseconds and is on a logarithmic scale. This boxplot is nearly identical to the boxplot for the constraint solving times for the automata models due to the practical irrelevance of the model counting time when combining it with the constraint solving time.

\subsubsection{Accuracy vs Performance Comparisons}

The comparisons of the accuracy and performance metrics reported by the evaluation are summarized in the following three subsections: model counting percentages difference versus cumulative constraint solving time in Section 6.1.3, model counting percentages difference versus model counting time in Section 6.1.3, and model counting percentages difference versus combined cumulative constraint solving and model counting time in Section 6.1.3. 


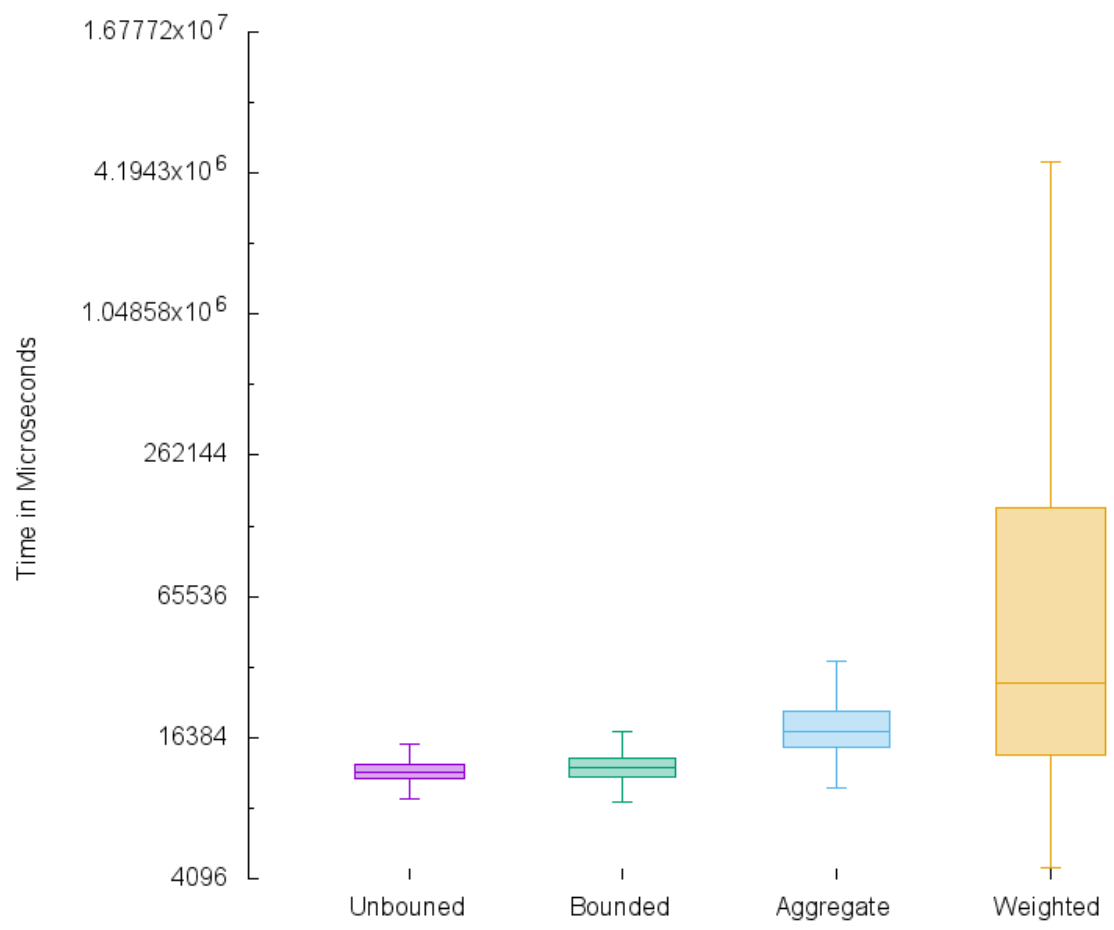

Figure 6.3: Boxplots of Combined Model Counting and Constraint Solving Times

\section{Accuracy vs Constraint Solving Performance}

Figure 6.4 shows a scatter plot for all four automata models where the $\mathrm{x}$-axis is the constraint solving time and the $y$-axis is the branch probability percentage difference, the $\mathrm{x}$-axis is on a logarithmic scale. The scatter plot shows the bounded automata model has the best balance of performance and accuracy where the bounded model is clustered just left of the aggregate model on the log scaled x-axis. This scatter plot also helps to illustrate just how many of the 542592 string constraints for each automata model result in large percentage differences. This was captured by the standard deviation measurements for each model, but the visualization in Figure 6.4 shows that achieving 100\% model counting accuracy will remain a difficult problem. 


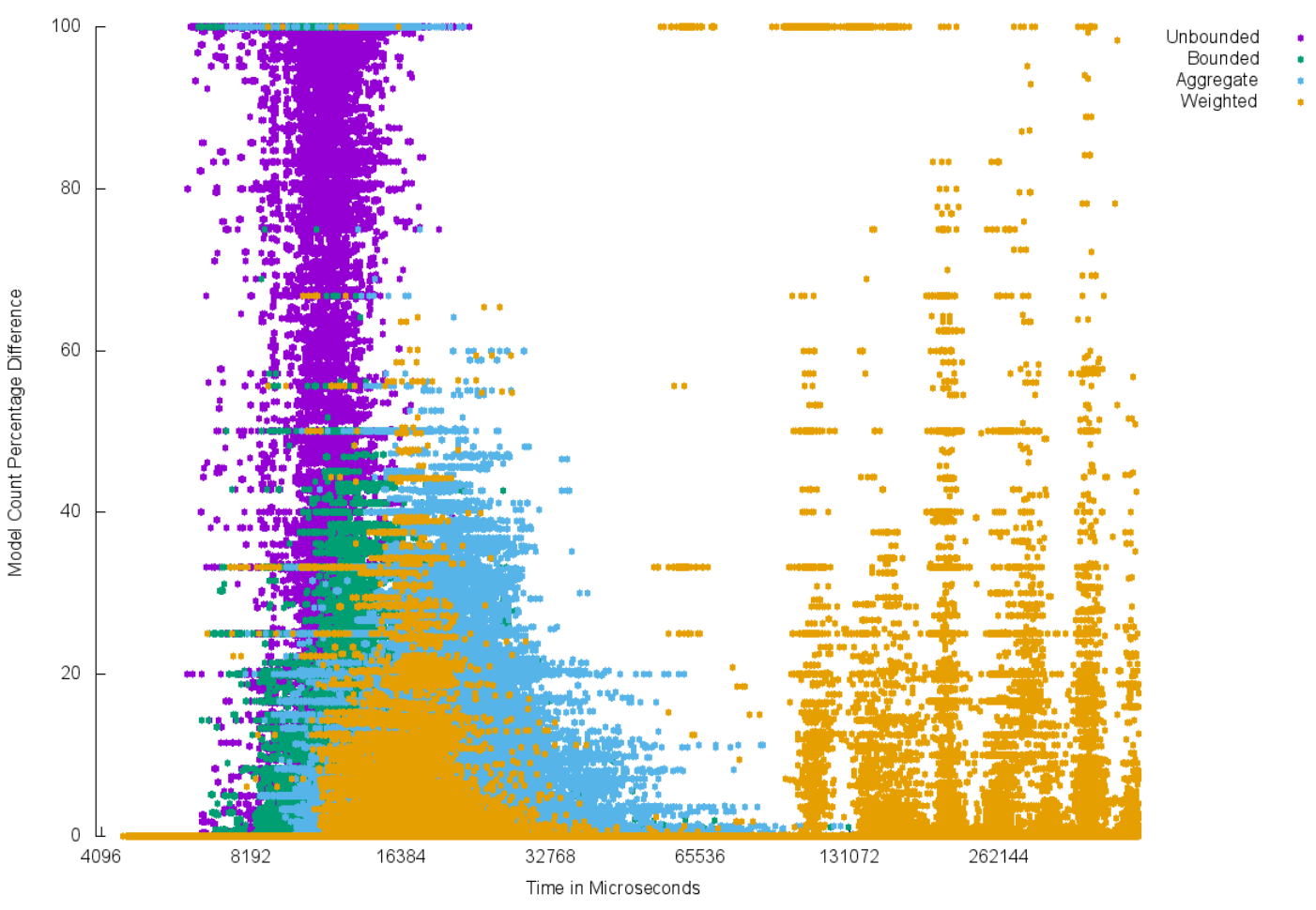

Figure 6.4: Scatterplot of Percentage Difference vs Constraint Solving Times

\section{Accuracy vs Model Counting Performance}

Figure 6.5 shows a scatter plot for all four automata models where the x-axis is the model counting time and the y-axis is the branch probability percentage difference. Because the model counting performance of each of the four automata models is practically equivalent, the plot of percentage difference vs model counting time mostly serves as as a visualization of the percentage differences between the automata models.

\section{Accuracy vs Combined Model Counting and Constraint Solving Perfor- mance}

Figure 6.6 shows a scatter plot for all four automata models where the x-axis is the combined constraint solving and model counting time and the y-axis is the branch 


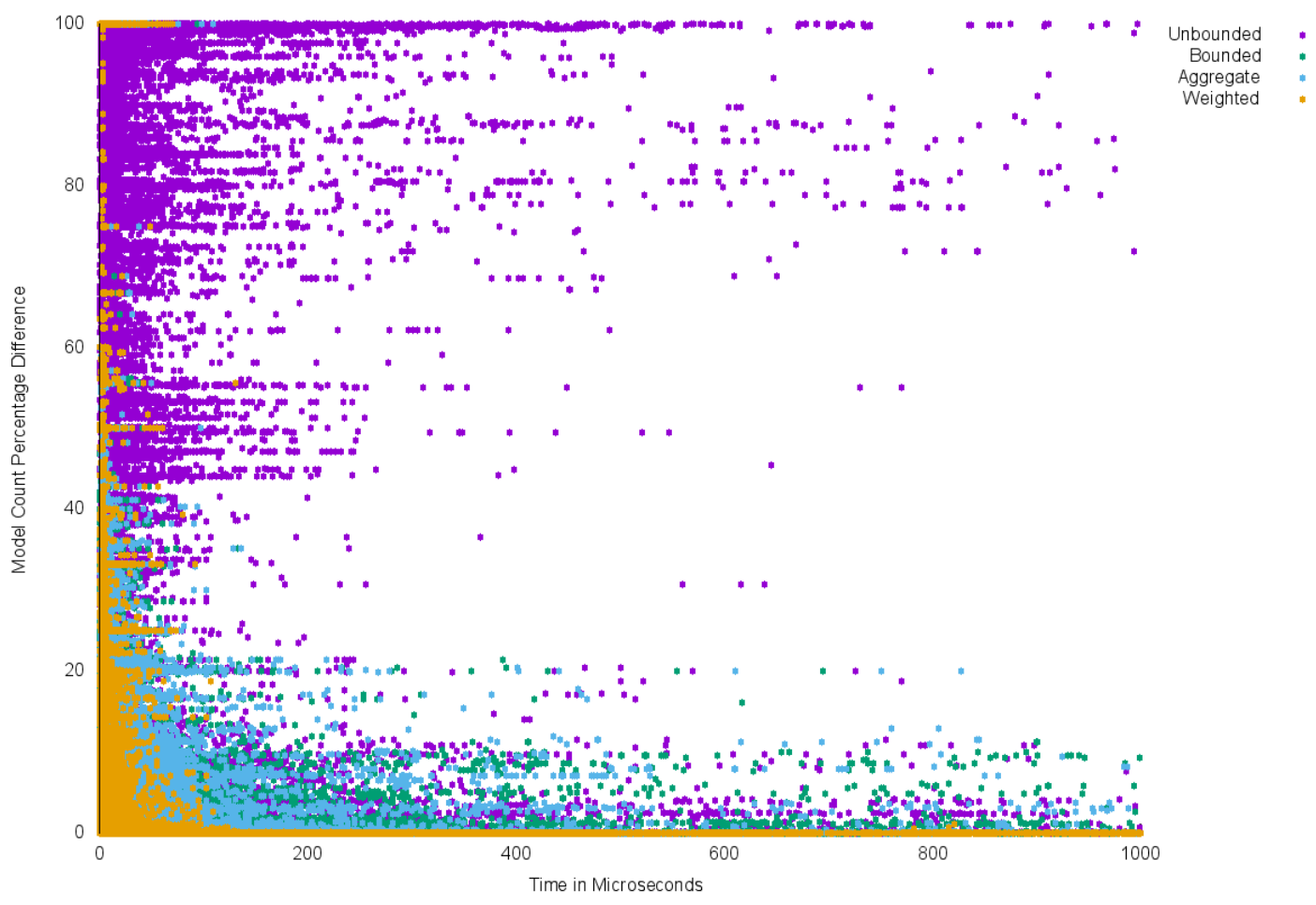

Figure 6.5: Scatterplot of Percentage Difference vs Model Counting Times

probability percentage difference, the $\mathrm{x}$-axis is on a logarithmic scale. Again, due to the practical irrelevance of model counting time when combined with constraint solving time, the scatter plot of percentage difference and combined time is nearly identical to the scatter plot of percentage difference and constraint solving time.

\subsection{Recommendations}

\begin{tabular}{|c|l|}
\hline Criteria & \multicolumn{1}{|c|}{ Model Comparison Relationship } \\
\hline Accuracy & Weighted $>$ Aggregate $\approx$ Bounded $>$ Unbounded \\
\hline Combined Performance & Unbounded $\approx$ Bounded $>$ Aggregate $>$ Weighted \\
\hline Balance of Accuracy and Performance & Bounded $>$ Aggregate $>$ Weighted $>$ Unbounded \\
\hline
\end{tabular}

Table 6.10: Solver Recommendations Based on Suitability 


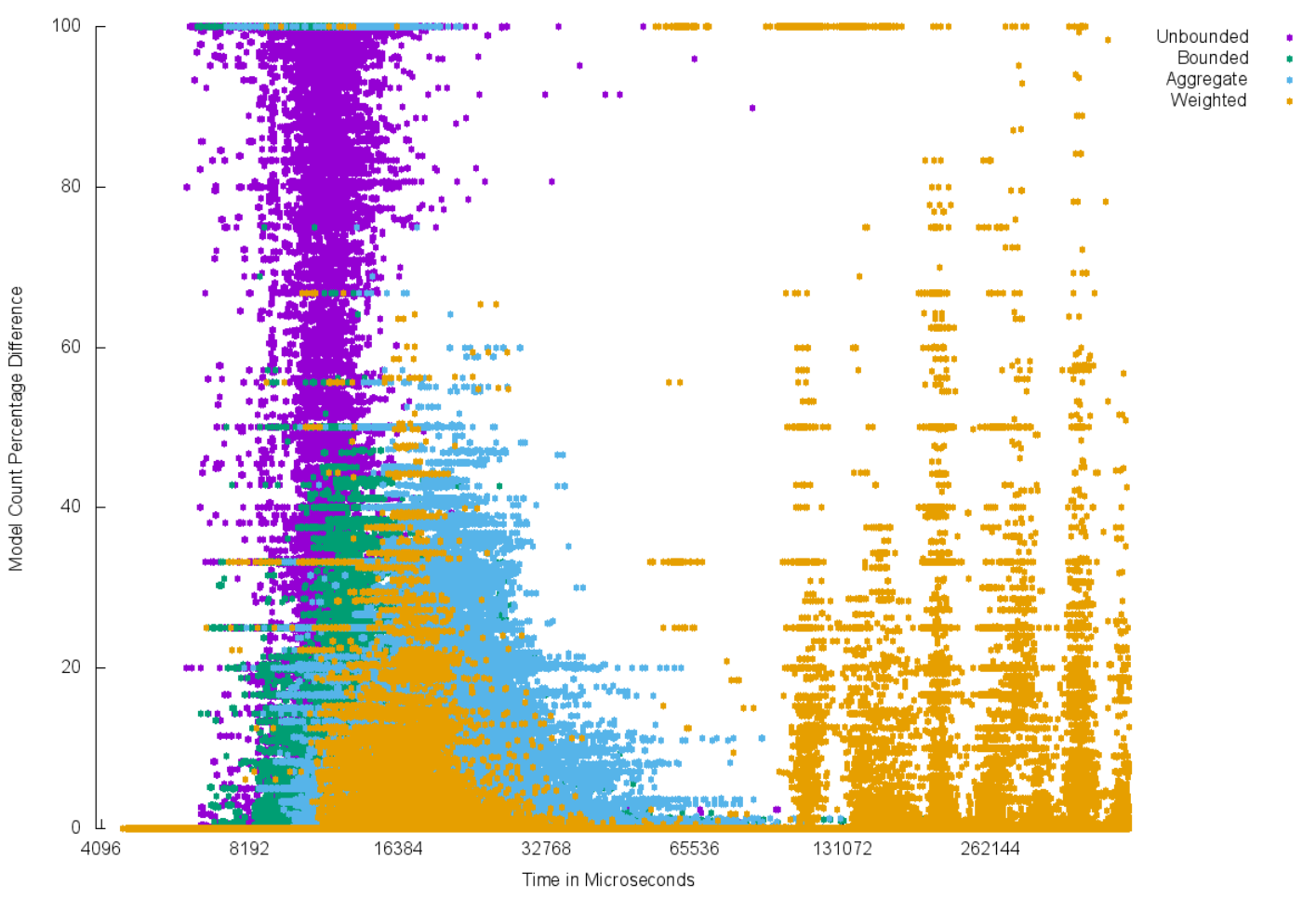

Figure 6.6: Scatterplot of Percentage Difference vs Combined Model Counting and Constraint Solving Times

Table 6.10 summarizes the accuracy and performance results discussed in Sections 6.1.1 and 6.1.2. The model with the best accuracy was the weighted automata model which maintained at least a 99\% branch agreement frequency across all independent variable data subsets and had a significantly smaller average percentage difference than the other automata models. The aggregate automata model had slightly smaller percentage difference metrics than the bounded model despite both models having practically equivalent branch choice frequencies. The unbounded automata model had significantly worse branch choice agreement frequency and significantly larger percentage differences than the other automata models. Both the unbounded and the bounded automata models have similar combined constraint solving and model counting times with the unbounded model performing slightly better than the bounded 
model. The aggregate model has a consistently longer combined times than either the unbounded or bounded models while the weighted model has significantly worse performance than any of the other three models.

When attempting to balance accuracy and performance, the bounded automata model has very good combined time and sufficient accuracy for most analyses with 99.3\% branch agreement frequency and only 1.02 average percentage difference. The aggregate model provides the second best balance between accuracy and performance with equivalent agreement frequency and slightly better percentage difference but the combined time of the aggregate model is significant enough compared to the bounded model that the bounded is clearly better balanced of the two models. While the weighted model is the most accurate of all the automata models, it is not enough of an accuracy improvement to overcome the significant combined time performance cost of the model compared to either the bounded or aggregate model. The unbounded model is has extremely poor accuracy when compared to each of the other automata models making it a worse choice than even the extremely slow weighted model.

\subsection{Threats to Evaluation Validity}

We have identified nine threats to the validity of the evaluation of the suitability of automata-based symbolic string models. These threats include two internal threats to validity and sLiteral external threats to validity.

\subsubsection{Internal Threats to Validity}

The identified internal threats to the validity of this evaluation include: the small size of the string alphabet and the small values for initial maximum string length. 
Small Size of the String Alphabet The small sizes of the string alphabet values in this evaluation appear to be a source of selection bias. This is a necessary choice in the evaluation because the time required to complete the evaluation grows quadratically as the alphabet size increases. This makes evaluation of larger alphabet sizes infeasible. To mitigate this risk, four alphabet sizes were chosen so that the effects on accuracy and performance for constraints with larger alphabets can can be extrapolated from the curve produced. Both the branch choice agreement and the percentage difference results indicate that model accuracy increases as the size of the string alphabet increases.

Small Values for the Initial String Length The small lengths chosen as initial maximum string length values in this evaluation appear to be another source of selection bias. This is also a necessary choice due to the evaluation time growing as the initial maximum length increases. As a result, small values are needed for the initial maximum string length if an evaluation to be feasible. As with the size of the string alphabet, the accuracy and performance suitability of the solvers for larger initial maximum string length values can be extrapolated from the curve produced for the four values evaluated. Both the branch choice agreement and the percentage difference results indicate that model accuracy increases as initial string length increases.

\subsubsection{External Threats to Validity}

The identified external threats to the validity of this evaluation include: the synthetic data set used in the evaluation, the evaluation of only reverse, concat, delete, and replace operations, using the char argument replace operation instead of String arguments, the use of only contains and equals predicates, the creation 
of Complex string types using only the contains predicate, the possible sub-optimal string operation modeling algorithms, the sub-optimal model counting algorithms, and the automaton models chosen for the evaluation.

Only reverse, concat, delete, and replace Operations Because only the reverse, concat, delete, and replace operations appear in the the string constraint graphs, the results of this evaluation may not apply for constraints with other string operations. However, observations from the SCSF test suite and made during early experimental testing show that each operation in a category has similar effects on the automata model count. Additionally, the string operation modeling algorithms for each category of operation have similar performance cost complexity. These two observed similarities indicate that the relative differences the evaluation reports between automata-based solvers are likely to hold for those operations not evaluated from evaluated categories.

replace with char Arguments Similar to the previous threat, using the char argument version of the replace operation appearing in the string constraint graphs without the String argument version also being included can mean the evaluation finding are not applicable to constraints which include the String argument version of the operation. The reason this threat to validity differs from the previous threat is the length alteration that can result from a String argument replace operation. Since this alteration can not occur for the char argument version, it would appear capable of producing different accuracy and performance results for subsequent constraints. However, exploratory testing shows that this does not occur for known string argument values. The use of unknown string argument values as used in the concat 
operation, the contains predicate, and the equals predicates is a topic for future exploration and is detailed in the Future Work section (6.5.2).

Only contains and equals predicates Because the contains and equals predicates are the only predicates included in the the Constraint Graphs, the results of the evaluation may not be generalizable to constraints with predicates which were not included. However, as with the string operations in the evaluation, the effects observed for automata model count using different predicates within both partial match and full match categories are similar enough that evaluating more than one predicate from either category was deemed redundant. This also applies to the performance effects of the different predicates where the algorithm complexity of the predicates within either category was similar enough that more than one predicate from either category was again deemed redundant.

Creating Complex Unknown String Type Using Only contains As a consequence of only using the contains operation to create complex unknown string types in the string constraint graphs, the evaluation findings may not apply to string constraints where complex unknown strings types may occur with different origins. The reason a complex unknown string type is needed as an initial string in the evaluation is due to the observed added vulnerability to such string types to over-approximation as well as collapses due to both subtractive and substitutive string operations. However, since automata models produced from contains operations have been proven to experience both the over-approximation and collapse issues, we feel complex unknown string types created using only the contains predicate is sufficient to characterize the accuracy and performance of automata-based symbolic 
string models.

Sub-optimal Operation and Predicate Simulation Algorithms The use of string operation and predicate simulation algorithms which are likely to be suboptimal can result in the evaluation results not being relevant for automata-based solvers using optimal operation modeling algorithms. However, because the SCSF tool implemented the same simulation algorithms for FSAs contained within unbounded, bounded, and aggregate automata models, the comparative accuracy and performance of these three automata models would be consistent with an evaluation using more optimized FSA algorithms. Additionally, because the weighted automata model uses string operation and predicate simulation algorithms which use WFSAs similarly to the corresponding FSA algorithms, the similar complexity of these WFSA algorithms allow comparisons to be made between weighted automata model accuracy and performance and the other automata models which would be consistent with an evaluation with more optimized WFSA algorithms.

Sub-optimal Model Counting Algorithms Similar to the previous threat, the use of sub-optimal model counting algorithms may result in the evaluation results not applying to solvers which use optimal model counting algorithms. However, the nearly identical computational complexity of the three utilized model counting algorithms MCUnbounded, MCBounded, and MCWEIGHTED allows the relationship between the four automata models regarding accuracy and performance to be consistent with evaluations using more optimal model counting algorithms.

Chosen Automata Models The choices of automaton models is the last identified threat to evaluation validity. It is possible that the results of this evaluation would 
not be applicable to either significantly modified versions of the four automaton models or entirely new automaton models. However, because the evaluation of automata models is intended to explore and illuminate the different accuracy problems suffered by automata models and how performance is affected by the presence of such problems, the four chosen automata models are sufficient to satisfy this intention. Since these accuracy issues arise from the unbounded automata model which is the automata model of choice in string analysis research, any other new or heavily modified automata models would need to address these same concerns as well.

\subsection{Conclusions}

Our analysis of the suitability of automata-based symbolic string models determined that none of the four proposed automata models were suitable to model string constraints without some amount inaccuracy. Whether or not a particular automata model is suitable for a particular analysis will largely depend on the accuracy and performance requirements of the analysis itself and we hope the results of our evaluation provide enough data to help others in making this determination. While this evaluation of suitability is dependent upon the particular analysis, the significant inaccuracies of the unbounded automata model makes it unsuitable for all but the most forgiving string analyses. Because the unbounded automata model is essentially the same model used in most ongoing quantitative string analysis research, it is important that unsuitability of the model in any practical quantitative analysis is known.

Some insights into the factors which affect automata model accuracy were observed in the evaluation of the four automata models. Both the branch choice agreement and percentage difference results demonstrate that the characteristics of the initial string 
do not have any significant effect upon the resulting accuracy metric. In fact, the trend of ever increasing accuracy as both the size of the string alphabet and the initial string length increases indicates that for much larger alphabet sizes and initial string lengths, such as those appearing in analyses of real world software programs, The inaccuracies seen by each of the automata models would be less likely to occur. The main source of inaccuracy for each of the automata models is the string operations and predicates, in particular the substitutive operations and the partial match predicates. Only the weighted automata model did not suffer from significant inaccuracy due to substitutive string operations in our evaluation because it was created to prevent substitutive string

collapses. However, even the weighted model had significant inaccuracy due to partial match predicates, although the magnitude of these inaccuracies were not as large as those seen for the other three automata models for partial match predicates.

From the results of this evaluation, we propose that a greater focus should be applied to three particular areas for automata-based symbolic string model research: improvements in multiset models, optimization of string operation simulation algorithms, and optimization of string predicate simulation algorithms.

\subsection{Possible Future Work}

While our analysis of the suitability of automata-based symbolic string models provides answers about what is required of a sufficiently suitable automata model, this analysis has raised many possibilities for future testing, additional evaluation enhancements, and other possible techniques to provide symbolic string models. 


\subsubsection{Additional testing}

We would like to expand the variety of string operations and predicates used in this evaluation to include addition injective, additive, subtractive, and substitutive operations as well as additional full match and partial match predicates. However, such an addition would require each additional operation and predicate to be simulated by an algorithm which would need to be created for the weighted automata model and possibly for the other three models if no such algorithm currently exists in the Java String Analyzer library.

We would also like to evaluate the string constraint graphs of real world software programs such as the open source software dataset evaluated by Kausler [21]. Unfortunately, such an evaluation would require either significant optimization of the string constraint solver oracle or the use of a super computer if not both. This would also require a significant rewrite of the solver oracle to process very large collections of strings without encountering memory errors as the current version would encounter for such large collections.

\subsubsection{Enhancements}

We would like add different automata models to the SCSF and evaluation to determine if some other choices about the design of the automata models could result in better accuracy or performance. One such change would be the use of factors and the strategy of restructuring aggregate automata models, we would like to explore other possible strategies to handle this problem without introducing the model count over-approximation discussed in Section 3.3.3. Another such change would be to test a version of the weighted automata model which does not utilize a sequence of 
WFSAs but instead uses only a single WFSA since the weighted-transitions should be sufficient to prevent subtractive collapses. While these are the only two known additional models we would like to evaluate, it would be very useful to evaluate other currently unknown automata models in the same way as the four currently implemented models.

We would also like to improve the algorithms required to solve and count the string constraints. While our evaluation ensured that the relative comparisons between each automata model was consistent due to the similar operation and predicate simulation algorithms, we would like to explore the accuracy and performance improvements that could be possible by optimizing these algorithms. One idea in particular would be to utilize concurrent processing for many of these algorithms where a process is applied for each transition or state in a collection. While no amount of this kind of optimization would allow constraint solving time to be similar to model counting time, such optimization would allow for much quicker quantitative string analyses.

Another area we would like to explore is the impacts upon accuracy of string operations and predicates with different combinations of arguments. While we explored some different operation and predicate argument configurations in our evaluation, we did not explore the full depth of such configurations. An example of such a set of argument configurations is seen for the SUBSTRING operation which can have four possible argument configurations: known start and end indices, a known start index but unknown end index, an unknown start index and unknown end index, and unknown start and end indices. Similar configurations exist for most of the different string operations and predicates and we would like to explore how these different configurations impact the accuracy and performance of a string analysis.

Finally, we would like to enhance our evaluation by adding support for mixed 
constraint string operations and predicates. This would allow us to evaluate the impacts upon accuracy and performance of mixed constraint operations and predicates. This evaluation could also include comparisons between different implementations of the symbolic models of the other data constraints in an attempt to find the best combination of symbolic string models and other data type symbolic models.

\subsubsection{Other}

One area of future work that we have shown to be of vital importance to quantitative string analysis is the need for multiset symbolic models. While we have attempted to create one such model with the weighted automata model, it is important to evaluate the advantages and disadvantages for creating and using multiset models for other commonly used symbolic string models such as bit-vectors and axiom-based models. Obviously a bit-vector can not be used since the use of only a single bit does not allow the representation of more than a single instance of an element in a set, but other vector based symbolic models could be created in the same manner that we created the weighted-transition finite state automaton from the finite state automaton. Also, the creation of multiset models should not be limited to symbolic string models either, multiset models are needed for all datatypes in quantitative analyses.

Another possible area of future research we can speculate about based on our evaluation is the use of machine learning models to predict both branch choice and global execution probability. By utilizing either our concrete string solver oracle or even an accuracy automata model such as the weighted model, a machine learning model could be trained to perform quantitative string analyses with the performance advantages enjoyed by most machine learning models. 


\subsection{Final Thoughts}

We hope that our evaluation of the suitability of finite-state automata to model string constraints in quantitative string analyses has provided useful information to guide both the selection of automata models in analyses and the direction of future string analysis research. We believe we proved the unsuitability of the commonly used unbounded automata-based symbolic string model for quantitative analyses to to it being significantly susceptible to accuracy errors. We believe we demonstrated the need to use multiset symbolic models such as the weighted automata-based symbolic string model in quantitative analyses. Finally, we believe we demonstrated the need for more research and optimization of the algorithms which simulate string operations and predicates for symbolic string models in quantitative analyses instead of research and optimization of model counting symbolic string models. 


\section{REFERENCES}

[1] Abdulbaki Aydin, Lucas Bang, and Tevfik Bultan. Automata-based model counting for string constraints. In International Conference on Computer Aided Verification, pages 255-272. Springer, 2015.

[2] Kent Beck. Test-driven development: by example. Addison-Wesley Professional, 2003.

[3] Nikolaj Bjørner, Nikolai Tillmann, and Andrei Voronkov. Path feasibility analysis for string-manipulating programs. In International Conference on Tools and Algorithms for the Construction and Analysis of Systems, pages 307-321. Springer, 2009.

[4] Mateus Borges, Antonio Filieri, Marcelo d'Amorim, Corina S Păsăreanu, and Willem Visser. Compositional solution space quantification for probabilistic software analysis. In ACM SIGPLAN Notices, volume 49(6), pages 123-132. ACM, 2014.

[5] Bernard Botella, Arnaud Gotlieb, and Claude Michel. Symbolic execution of floating-point computations. Software Testing, Verification and Reliability, 16(2):97-121, 2006.

[6] Janusz A Brzozowski. Canonical regular expressions and minimal state graphs for definite events. Mathematical theory of Automata, 12(6):529-561, 1962.

[7] Aske Simon Christensen, Anders Møller, and Michael I Schwartzbach. Precise analysis of string expressions. In International Static Analysis Symposium, pages 1-18. Springer, 2003.

[8] David Clark, Sebastian Hunt, and Pasquale Malacaria. A static analysis for quantifying information flow in a simple imperative language. Journal of Computer Security, 15(3):321-371, 2007.

[9] Olivier Coudert, Christian Berthet, and Jean Christophe Madre. Verification of synchronous sequential machines based on symbolic execution. In International Conference on Computer Aided Verification, pages 365-373. Springer, 1989. 
[10] Isil Dillig, Thomas Dillig, Alex Aiken, and Mooly Sagiv. Precise and compact modular procedure summaries for heap manipulating programs. In $A C M S I G$ PLAN Notices, volume 46(6), pages 567-577. ACM, 2011.

[11] Michael Emmi, Rupak Majumdar, and Koushik Sen. Dynamic test input generation for database applications. In Proceedings of the 2007 international symposium on Software testing and analysis, pages 151-162. ACM, 2007.

[12] Asger Feldthaus and Anders Møller. The Big Manual for the Java String Analyzer. Department of Computer Science, Aarhus University, November 2009. Available from http://www.brics.dk/JSA/.

[13] Antonio Filieri, Corina S Păsăreanu, and Willem Visser. Reliability analysis in symbolic pathfinder. In Proceedings of the 2013 International Conference on Software Engineering, pages 622-631. IEEE Press, 2013.

[14] Martin Fowler. Givenwhenthen, August 2013.

[15] Erich Gamma. Design patterns: elements of reusable object-oriented software. Pearson Education India, 1995.

[16] Jaco Geldenhuys, Matthew B Dwyer, and Willem Visser. Probabilistic symbolic execution. In Proceedings of the 2012 International Symposium on Software Testing and Analysis, pages 166-176. ACM, 2012.

[17] Indradeep Ghosh, Nastaran Shafiei, Guodong Li, and Wei-Fan Chiang. Jst: An automatic test generation tool for industrial java applications with strings. In Proceedings of the 2013 International Conference on Software Engineering, pages 992-1001. IEEE Press, 2013.

[18] Pieter Hooimeijer and Margus Veanes. An evaluation of automata algorithms for string analysis. In Verification, Model Checking, and Abstract Interpretation, pages 248-262. Springer, 2011.

[19] Pieter Hooimeijer and Westley Weimer. A decision procedure for subset constraints over regular languages. In ACM Sigplan Notices, volume 44(6), pages 188-198. ACM, 2009.

[20] John Hopcroft. An n $\log \mathrm{n}$ algorithm for minimizing states in a finite automaton. In Theory of machines and computations, pages 189-196. Elsevier, 1971.

[21] Scott Kausler. Evaluation of string constraint solvers using dynamic symbolic execution. PhD thesis, Boise State University, 2014. 
[22] Sarfraz Khurshid, Corina S Păsăreanu, and Willem Visser. Generalized symbolic execution for model checking and testing. In International Conference on Tools and Algorithms for the Construction and Analysis of Systems, pages 553-568. Springer, 2003.

[23] Adam Kiezun, Vijay Ganesh, Philip J Guo, Pieter Hooimeijer, and Michael D Ernst. Hampi: a solver for string constraints. In Proceedings of the eighteenth international symposium on Software testing and analysis, pages 105-116. ACM, 2009.

[24] James C King. Symbolic execution and program testing. Communications of the $A C M, 19(7): 385-394,1976$.

[25] Daniel Kröning, Philipp Rümmer, and Georg Weissenbacher. A proposal for a theory of finite sets, lists, and maps for the smt-lib standard. In Informal proceedings, 7th International Workshop on Satisfiability Modulo Theories at $C A D E$, volume 22, page 24, 2009.

[26] Christopher Kruegel, Engin Kirda, Darren Mutz, William Robertson, and Giovanni Vigna. Automating mimicry attacks using static binary analysis. In Proceedings of the 14th conference on USENIX Security Symposium-Volume 14, pages 11-11. USENIX Association, 2005.

[27] Loi Luu, Shweta Shinde, Prateek Saxena, and Brian Demsky. A model counter for constraints over unbounded strings. In ACM SIGPLAN Notices, volume 49(6), pages 565-576. ACM, 2014.

[28] Edward F Moore. Gedanken-experiments on sequential machines. Automata studies, 34:129-153, 1956.

[29] Quoc-Sang Phan, Pasquale Malacaria, Corina S Păsăreanu, and Marcelo d'Amorim. Quantifying information leaks using reliability analysis. In Proceedings of the 2014 International SPIN Symposium on Model Checking of Software, pages 105-108. ACM, 2014.

[30] Quoc-Sang Phan, Pasquale Malacaria, Oksana Tkachuk, and Corina S Păsăreanu. Symbolic quantitative information flow. ACM SIGSOFT Software Engineering Notes, 37(6):1-5, 2012.

[31] Michael O Rabin and Dana Scott. Finite automata and their decision problems. IBM journal of research and development, 3(2):114-125, 1959.

[32] Gideon Redelinghuys, Willem Visser, and Jaco Geldenhuys. Symbolic execution of programs with strings. In Proceedings of the South African Institute for 
Computer Scientists and Information Technologists Conference, pages 139-148. ACM, 2012.

[33] Koushik Sen, Darko Marinov, and Gul Agha. Cute: a concolic unit testing engine for c. In ACM SIGSOFT Software Engineering Notes, volume 30(5), pages 263-272. ACM, 2005.

[34] Daryl Shannon, Sukant Hajra, Alison Lee, Daiqian Zhan, and Sarfraz Khurshid. Abstracting symbolic execution with string analysis. In Proceedings of the Testing: Academic and Industrial Conference Practice and Research TechniquesMUTATION, pages 13-22. IEEE Computer Society, 2007.

[35] Geoffrey Smith. On the foundations of quantitative information flow. In International Conference on Foundations of Software Science and Computational Structures, pages 288-302. Springer, 2009.

[36] Takaaki Tateishi, Marco Pistoia, and Omer Tripp. Path-and index-sensitive string analysis based on monadic second-order logic. ACM Transactions on Software Engineering and Methodology (TOSEM), 22(4):33, 2013.

[37] Fang Yu, Muath Alkhalaf, and Tevfik Bultan. Stranger: An automata-based string analysis tool for php. In Tools and Algorithms for the Construction and Analysis of Systems, pages 154-157. Springer, 2010.

[38] Fang Yu, Muath Alkhalaf, Tevfik Bultan, and Oscar H Ibarra. Automata-based symbolic string analysis for vulnerability detection. Formal Methods in System Design, 44(1):44-70, 2014.

[39] Fang Yu, Tevfik Bultan, Marco Cova, and Oscar H Ibarra. Symbolic string verification: An automata-based approach. In Model Checking Software, pages 306-324. Springer, 2008.

[40] Fang Yu, Tevfik Bultan, and Oscar H Ibarra. Symbolic string verification: Combining string analysis and size analysis. In Tools and Algorithms for the Construction and Analysis of Systems, pages 322-336. Springer, 2009.

[41] Fang Yu, Tevfik Bultan, and Oscar H Ibarra. Relational string verification using multi-track automata. International Journal of Foundations of Computer Science, 22(08):1909-1924, 2011.

[42] Yunhui Zheng, Xiangyu Zhang, and Vijay Ganesh. Z3-str: A z3-based string solver for web application analysis. In Proceedings of the 2013 9th Joint Meeting on Foundations of Software Engineering, pages 114-124. ACM, 2013. 